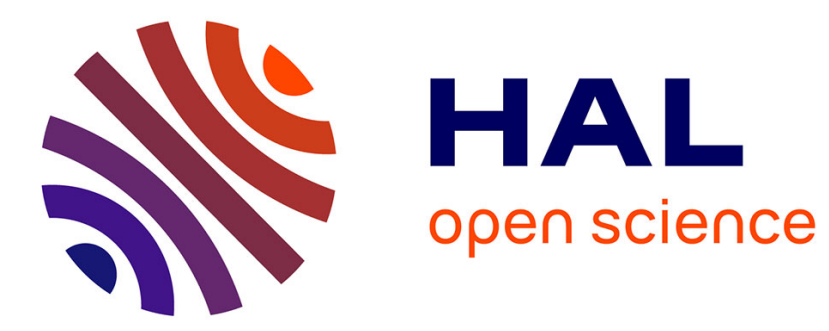

\title{
Grain boundaries in high temperature superconducting ceramics
}

Jeanne Gilberte Ayache

\section{To cite this version:}

Jeanne Gilberte Ayache. Grain boundaries in high temperature superconducting ceramics. Philosophical Magazine, 2006, 86 (15), pp.2197-2243. 10.1080/14786430600640494 . hal-00513675

\section{HAL Id: hal-00513675 \\ https://hal.science/hal-00513675}

Submitted on 1 Sep 2010

HAL is a multi-disciplinary open access archive for the deposit and dissemination of scientific research documents, whether they are published or not. The documents may come from teaching and research institutions in France or abroad, or from public or private research centers.
L'archive ouverte pluridisciplinaire HAL, est destinée au dépôt et à la diffusion de documents scientifiques de niveau recherche, publiés ou non, émanant des établissements d'enseignement et de recherche français ou étrangers, des laboratoires publics ou privés. 


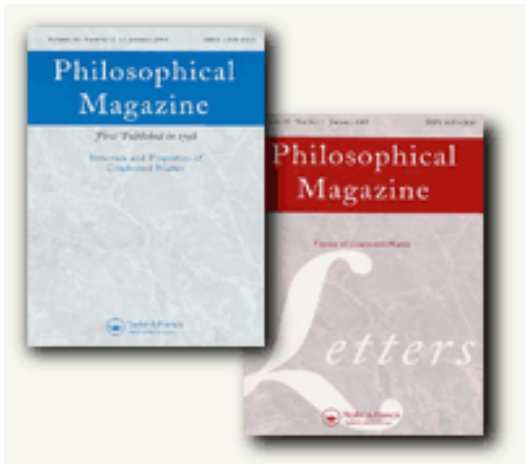

\section{Grain boundaries in high temperature superconducting ceramics}

\begin{tabular}{|r|l|}
\hline Journal: & Philosophical Magazine \& Philosophical Magazine Letters \\
\hline Manuscript ID: & TPHM-05-Jun-0285.R1 \\
\hline Journal Selection: & Philosophical Magazine \\
\hline Date Submitted by the \\
Author: & 13-Dec-2005 \\
\hline Complete List of Authors: & AYACHE, Jeanne; CNRS, CSNSM-IN2P3 \\
\hline Keywords: & Superconducting materials, grain boundaries \\
\hline Keywords (user supplied): & \\
\hline &
\end{tabular}

\section{S) ScholaroNE \\ Manuscript Central}




\title{
Grain boundaries in high temperature superconducting ceramics
}

\author{
Jeanne AYACHE \\ Centre de Spectroscopie Nucléaire et Spectrométrie de Masse \\ CSNSM-CNRS-IN2P3, Bâtiment 108, 91405 Orsay Campus, France. \\ ayache@csnsm.in2p3.fr, Tel :33 01691552 19, Fax : 330169155268
}

\begin{abstract}
The aim of this paper is to review the different types of grain boundaries found in $\mathrm{YBa}_{2} \mathrm{Cu}_{3} \mathrm{O}_{7-\mathrm{d}}$ superconducting ceramics which have been investigated over the past decade. A variety of samples covering a wide range of current density Jc values are examined including: classical bulk ceramics, melt-textured bulk ceramics, bulk and thin film bicrystals with specific orientations, thin films and single crystals. From electronic coupling, Jc is expected and found to decrease with increasing grain boundary (GB) misorientation. From the magnetic behaviour, the critical current density $\mathrm{Jc}(\mathrm{H})$ is expected to increase with the number of pinning centres. The properties of the various types of GB's are discussed. All the studies reviewed lead to the conclusion that grain boundaries in YBCO materials cannot be understood without specific information about their local composition, structure and electronic state. They confirm that research on bicrystals is crucial for high-temperature superconductor development.
\end{abstract}

\section{Introduction}

Before 1987, most of the superconducting materials were characterized by a low critical temperature ( $\mathrm{Tc}$ ) close to liquid helium temperature $(4.2 \mathrm{~K})$. They were metal or intermetallic compounds $\left(\mathrm{Nb}_{3} \mathrm{Ge}\right.$ for instance with $\left.\mathrm{Tc}=23 \mathrm{~K}\right)$. New high temperature superconducting cuprates (HTSC) with high Tc (above liquid nitrogen at 77K) based on lanthanides (Y, Ho, Nd for example), baryum and copper oxides were then discovered and found useful for industrial applications. Among them, $\mathrm{YBa}_{2} \mathrm{Cu}_{3} \mathrm{O}_{7-\mathrm{d}}(\mathrm{YBCO})$ ceramics, with a $\mathrm{Tc}$ close to $90 \mathrm{~K}$, was synthesized and extensively studied [1], [2]. At present, other compounds like Bi-Sr-Ca-Cu-O $(\mathrm{Tc}=108 \mathrm{~K})[2]$ and $\mathrm{Tl}-\mathrm{Sr}-\mathrm{Ca}-\mathrm{Cu}-\mathrm{O}(\mathrm{Tc}=135 \mathrm{~K})[3]$ have become well known.

All these HTSC's are complex layered copper oxides, separated by $\mathrm{CuO}_{2}$ layers which are 
mainly responsible for the superconductivity. One of the applications of the superconducting state makes use of the variation of the electrical resistance as a function of temperature, i.e. at the critical temperature the resistivity drops suddenly to zero, due to condensation and coupling between the superconducting electron Cooper pairs. The critical transport current density (Jc) property dictates the type of application which can be achieved with a particular superconductor. The value of Jc is proportional to the percolation paths, and depends strongly on the quality of grain boundaries (GB's) in these ceramics. Understanding and controlling GB's is found to be crucial for bulk material and thin film applications. Among the structural defects formed in ceramics, GB's are especially interesting, because they act as Josephson junctions [4], [5] and thus are good candidates for elucidating the mechanism of superconductivity. 


\section{Superconducting properties and their consequences for applications}

A determining characteristic for HTSC's is their magnetic behaviour at the critical temperature Tc. They are characterized by the coexistence of superconducting and nonsuperconducting areas in the sample between the two critical magnetic fields $\mathrm{Hc}_{1}$ and $\mathrm{Hc}_{2}$. Such zones, resulting from the penetration of the applied magnetic field $\left(\mathrm{H}_{\text {appl }}\right)$, are described as vortex centres. Each of them carries a magnetic flux H. As a result, supercurrents are produced around each vortex, leading to the expulsion of the magnetic flux (Meissner effect). The resulting critical current density Jc is proportional to the number of pinning centers; the higher the number of vortices, the higher is the critical density Jc. Consequently, structural defects like point defects, dislocations, GB's and interfaces, will act as pinning centers and favour the increase of Jc due to the pinning energy dissipation. This property will compensate for the Jc limiting effect of weak-link coupling at grain boundaries. Consequently, GB Jc 
values can be deduced from hysteresis loop magnetic measurements at low magnetic fields (see section 5).

Generally, properties of devices based on GB Josephson junctions are strongly influenced by the GB microstructure, due to the tunneling phenomenon itself, and also due to the presence of a thin interface layer between two superconductors. This layer can have either normal metal (S/N/S), insulating (S/I/S) or weakly superconducting properties. The superconducting state of Cooper pair electrons across the interface is characterized by a pseudo-wave function which depends on the phase $\Delta \mathrm{F}$ of the superconducting order parameter $\Delta(\mathrm{OP})$. The Josephson effect is very sensitive to the phase shift $\Delta \mathrm{F}$ of this order parameter $\left(\mathrm{J}_{\mathrm{c}}=\mathrm{J}_{1} \sin \Delta \mathrm{F}\right)$. For conventional low Tc superconductors, the OP is characterized by «s-wave symmetry » with isotropic properties. As a result, phase and current are both constant. For HTSC's, the OP presents a «d-wave symmetry » characterized by anisotropic properties and an internal phase whose value depends on crystallographic orientation, in the same way as the current Ic = $\mathrm{f}(\Delta \mathrm{F})$ does. This latter is maximum only along some crystallographic orientations and null outside. This characteristic is fundamental for the understanding of GB properties. Figure 2 schematizes a S/I/S junction with its characteristics for both types of superconducting mechanisms. In low-Tc superconductors (LTS) the coherence length $\xi=10^{2}-10^{3} \mathrm{~nm}$ is large and isotropic, compared to HTSC's for which $\xi$ is of the order of the interatomic d-spacing $(\xi \approx 1 \mathrm{~nm})$ and anisotropic. Such a small value makes the OP amplitude and phase very sensitive to the effect of a local structural change. Consequently, any change in GB misorientation on the atomic scale will strongly affect the coupling between the two grains, leading to a local decrease of Jc, as shown for bicrystal thin films (see section 8).

Thus, it is the grain boundary misorientation which controls the macroscopic Jc characteristic, due to decreasing coupling between the two grains. The higher the misorientation angle, the lower the coupling is expected to be. Hence, Jc is expected to have a defined value for a specific misorientation between the two grains. High angle grain boundaries (HAGB) act as Josephson junction weak-links. Consequently, a HAGB in the microstructure introduces a network of weak-links (corresponding to regions of reduced Jc ) into a superconducting path which otherwise would have Jc characteristics appropriate for applications. Nevertheless, it was shown that some HAGB's are strongly coupled, leading to rather good properties as in the BSCCO system, although YBCO ceramics show a weak-link character with locally oxygen deficient GB areas. It has become increasingly clear from electromagnetic measurements and microstructural studies that weak-link boundaries are heterogeneous on various scales. The characteristic properties of Josephson junctions, like weak-links, include a strongly magnetic field dependent $\mathrm{Jc}(\mathrm{H})$ which can decrease by more than an order of magnitude for weak fields 
of just a few (1-10) millitesla. Such $\mathrm{Jc}(\mathrm{H})$ characteristics clearly pose a serious problem for magnetic applications (energy storage systems, motors, generators,...). As a result, a great number of HAGB's represent an obstacle to the development of bulk materials for such applications. Improvement of the Jc limiting effect of weak-link coupling at grain boundaries can be achieved, either by improving the Jc of individual grain boundaries, or by texturing the materials in order to reduce the number of weak-link grain boundaries in the current path. The high sensitivity of Jc to weak magnetic fields, at grain boundary Josephson junctions, enables HTSC applications in electronic materials and particularly in thin films (squids).

The anisotropy of coherence length values in the YBCO superconducting plane. $\xi_{\mathrm{ab}}=1-2 \mathrm{~nm}$ ) and in the $\mathrm{c}$-axis direction $\left(\xi_{\mathrm{c}}=0.3 \mathrm{~nm}\right)$ leads to a dependence of various superconducting properties on the crystallographic orientation of the sample. Based on the $d_{x 2-y 2}$ symmetry [7], [8] of the superconducting order parameter, a strong coupling is expected along [100] and [103] orientations, while [001] and [110] will present a weak coupling. Thus, as schematized in figure 3, only some orientations will carry the supercurrent. Among them, GB's containing (001) superconducting planes will be the most favourable.

From electronic coupling, it can be expected that Jc will decrease with increasing GB misorientation. This effect is used to study Josephson junction applications based on thin films. From the magnetic behaviour, the critical current density $\mathrm{Jc}(\mathrm{H})$ is expected to increase with the number of pinning centres, such as structural defects (point defects, dislocations, twins, clean interfaces). This particular property is used for high current transport applications in bulk ceramics. Among these cuprates, YBCO is the one that has been most extensively studied in bulk ceramics, thin films and heterostructures. Because of its particular interest, YBCO has been chosen as a model for understanding structure and superconductivity mechanisms.

The aim of this paper is to review the different types of grain boundary configurations and properties found in the YBCO system for bulk ceramics, melt-textured ceramics, thin films, bicrystals and single crystals used in the field of superconduction research and applications.

\section{3. $\mathrm{YBaCuO}$ system properties and their consequences on grain boundary structure}

\subsection{Structure and properties}

YBCO superconducting ceramics, like almost all HTSC, have a complex layered structure of perovskite blocks containing one or a few $\mathrm{CuO}_{2}$ layers which are currently considered to be mainly responsible of the superconductivity. In these layers, the copper atoms form a square network, whereas the oxygen atoms are placed along the copper-oxygen chains (fig. 3a). As 
$\mathrm{YBa}_{2} \mathrm{Cu}_{3} \mathrm{O}_{7-\mathrm{d}}$ has an orthorhombic structure based on that of perovskite oxides, it belongs to the class of compounds displaying, for copper atoms, a mixed valence which leads to a flexible chemistry. Another particular aspect of this system is the possible variation in cationic and oxygen stoichiometry.

The formation of the YBCO superconducting phase (also called the (123) phase referring to the cationic content in $\mathrm{Y}, \mathrm{Ba}$, and $\mathrm{Cu}$ atoms) involves a second order reaction transforming the high temperature tetragonal insulating $\mathrm{YBa}_{2} \mathrm{Cu}_{3} \mathrm{O}_{6}$ phase into the low temperature orthorhombic superconducting structure. The crystallographic structure of YBCO strongly depends on oxygen stoichiometry. The lower $\mathrm{O}_{\mathrm{d}}$, the higher is Tc. Its maximum value is obtained for an oxygen deficiency $d=0.03$. For $d=1$, the structure becomes tetragonal corresponding to an insulating material. Within this range, crystal structure and physical properties undergo changes. Two orthorhombic structures have been characterized, called "Ortho I" and "Ortho II". The first one refers to the fully oxygenated superconducting phase. The second one contains vacancies along the $\mathrm{O}-\mathrm{Cu}-\mathrm{O}$ chains and particularly one oxygen vacancy every two structural unit cells. The first one corresponds to an oxygen content in the range $\mathrm{O}_{7}-\mathrm{O}_{6.9}$ with a critical $\mathrm{Tc}$ remaining at $91 \mathrm{~K}$. The second one is characterized by an oxygen content of $\mathrm{O}_{6.6}$ or $\mathrm{O}_{6 . .7}$, for which Tc drops to a plateau at $60 \mathrm{~K}$ and finally to zero between $\mathrm{O}_{6.5}$ and $\mathrm{O}_{6.4}$. These various oxygen concentrations and related YBCO structures are summarized in Table 1 . For special oxygen contents $(0.15<\mathrm{d}<0.6)$, various types of oxygen vacancy ordering occur. They have been extensively studied by Transmission Electron Microscopy (TEM) and electron diffraction patterns by Alario-Franco et al [9, 10]. Other authors $[11,12]$ give numerous details on oxygen vacancy ordering near $0_{6.5}$. A review of YBCO and its derived structures can be found in reference [13]. These experimental results are in good agreement with theoretical calculations reported by Ceder [14] and Matic et al [15] and more recent studies based on first principle calculations which predict all possible oxygen vacancy orderings [16].

Figure 4 shows that lattice parameters change as a function of oxygen deficiency $\mathrm{d}$ for various oxygen partial pressures [6]; note the change of the transformation temperature from $798 \mathrm{~K}$ to 943K. This corresponds to an oxygen gain along the $\mathrm{Cu}-\mathrm{O}$ chains of the tetragonal structure (fig.3 and fig.4). It generates an increasing strain in the crystal due to the simultaneous increase of lattice parameter $\underline{b}$ and decrease of lattice parameter $\underline{a}$. Twins are formed and induce stress relaxation. The higher the number of twins formed, the higher is the stress. Twins are grain boundaries of special symmetry lower than that of the single crystal. Due to their origin, they are found in all superconducting YBCO crystals, bulk ceramics, melttextured ceramics and thin films. Their presence increases Jc inside each grain, because they 
act as pinning centres for the vortices. Twins are not the subject of this review and will not be described here. 


\subsection{Phase diagram and stoichiometry}

The important characteristic in the $\mathrm{Y}-\mathrm{Ba}-\mathrm{Cu}-\mathrm{O}$ system is the non-congruent melting behaviour of the $\mathrm{YBa}_{2} \mathrm{Cu}_{3} \mathrm{O}_{7-\mathrm{d}}$ compound which favours the formation of secondary phases such as $\mathrm{CuO}$, $\mathrm{Y}_{2} \mathrm{O}_{3}, \mathrm{BaO}, \mathrm{BaCuO}_{2}$ and $\mathrm{Y}_{2} \mathrm{BaCuO}_{5}$. The cationic non-stoichiometric (123) phase was observed in many RBCO ceramics, as well as secondary phases with specific properties. An understanding of the phase diagram has been mainly sought in order to determine the conditions of formation of the (123) phase and that of the secondary phases which also affect the physical properties of the bulk and of the interfaces. These secondary phases are formed outside of the thermodynamic growth conditions of the (123) superconducting phase. Cationic non-stoichiometry can be understood from the complex Y-Ba-Cu-O ternary phase diagram associated to the $\mathrm{BaO}-\mathrm{Y}_{2} \mathrm{O}_{3}-\mathrm{CuO}$ system (fig. 5). The diagram contains different domains of coexistence of solid-solid and solid-liquid phases. It shows the most likely domains for non-superconducting secondary phases to be (132), (211), beside the (123) phase. Among the secondary phases formed out of equilibrium, one may find barium, copper oxides or deficiency phases which are not superconducting such as (234), (235), and (113) [17], [18] and [19]. These authors observed in various ceramics synthesized by classical sintering, like $\mathrm{RBaCuO}(\mathrm{R}=\mathrm{Y}$ and $\mathrm{Ho})$, a large variety of cationic non-stoichiometric ceramics. The (123) superconducting phase can be formed either by solid-solid diffusion (close to the domain of the (132) phase) or by solid-liquid diffusion in the region of the (211) phase. Depending on thermodynamic conditions, the resulting (123) phase will have specific microstructures and properties. At high temperature, the YBCO phase decomposes (above 1233K), due to noncongruent melting. Depending on oxygen partial pressure, solid-solid diffusion takes place between $1193 \mathrm{~K}$ and $1233 \mathrm{~K}$. Grain growth governed by solid-solid atomic diffusion should lead to clean grain boundaries. Between the domain of $\mathrm{BaCuO}_{2}-\mathrm{CuO}$ and the (211) region, there is formation of a liquid with the diffusion governed by a liquid-solid process. At still higher temperatures near the peritectic reaction $(\mathrm{T}=1288 \mathrm{~K}$ in atmospheric conditions) $\mathrm{YBCO}$ decomposes in a liquid-rich phase containing $\mathrm{CuO}, \mathrm{BaCuO}_{2}$ and (211) precipitates. During this step, solid-liquid diffusion occurs leading to dissolution of the (211) precipitates into the liquid. This reaction is used to grow melt-textured ceramics. Grain growth governed by solidliquid diffusion can lead to grain boundaries with secondary phases due to recrystallization of the liquid. This will be detailed in the section dealing with grain boundaries in melt-textured ceramics (see section 6).

The role of oxygen partial pressure should also be stressed in connection with the structural phase diagram. This diagram highlights the effect of oxygen partial pressure and of oxygenation temperature on the formation of the (123) oxygen stoichiometric phase, as 
reported in figure 6. In particular, it shows the narrowness of the domain in which the optimum oxygen content for the (123) phase is achieved. It can be noticed that both the oxygen content at the orthorhombic-tetragonal transition and the transition temperature decrease with decreasing oxygen partial pressure [6].

Finally, the complexity of this system makes intrinsically difficult the formation of a single YBCO phase with oxygen and cationic stoichiometry. Improving YBCO growth has led to a better knowledge of the structures and superconducting properties. As a consequence, an increasing number of applications have been developed with bulk ceramics (for high current transport), thin films (electronic device applications, squids) and heterostructures (ferroelectric device applications, hard memories, ...).

\section{Grain boundaries in bulk ceramics}

Conventional ceramic sintering leads to a current density with a maximum value $\mathrm{Jc}=10^{3} \mathrm{~A} / \mathrm{cm}^{2}$ at $77 \mathrm{~K}$ (fig. 1) which is one thousand times less than that obtained for thin films and single crystals $\left(\mathrm{Jc}=10^{6} \mathrm{~A} / \mathrm{cm}^{2}\right)$. This large difference has various origins :

(i) The strong dependence of Jc on crystallographic orientation which is due to the intrinsic anisotropic properties of the YBCO structure;

(ii) The oxygen content due to oxygen self-diffusion;

(iii) The oxygen content dependence on GB misorientation;

(iv) The quality of the grain boundaries, either clean or damaged;

(v) Last but not the least, the type of processing used to make bulk samples ready for applications and leading to high quality GB's.

All these parameters are interacting simultaneously inside the grains and for each GB in the ceramic. Therefore, Jc results from a combination of these phenomena.

\subsection{Oxygen diffusion as the limiting step for grain boundary properties in polycrystals}

An important challenge for obtaining good superconducting properties is to control the elaboration process in order to reach the maximum oxygen content $\mathrm{O}_{6.9}-\mathrm{O}_{7}$. This problem can be dealt with by considering oxygen self-diffusion in ceramics. Sabras et al [20] investigated oxygen self-diffusion and its effects on grain boundary oxygen content by means of Secondary Ion Mass Spectroscopy (SIMS). From diffusion profiles using oxygen $\mathrm{O}^{18}$ as a tracer and from oxygen ion images, these authors found a heterogeneous distribution of the tracer in the material, as well as an influence of microcracks on oxygen diffusion. On polycrystalline ceramics exhibiting various grains sizes and an inhomogeneous grain size distribution, they find an oxygen diffusion coefficient along grain boundaries of the same 
order as in the bulk. Figure 7 depicts these experimental results and those found in the literature. Figure 7a shows oxygen diffusion depth profiles on a sample polished according to a small angle $\left(\alpha=1.56^{\circ}, \mathrm{T}=823 \mathrm{~K}, \mathrm{pO}_{2}=22 \mathrm{kPa}\right)$. It exhibits the presence of microcracks in the ceramic. In Figure $7 \mathrm{~b}$ an ion image obtained from $\mathrm{O}^{18}$ species shows the heterogeneous distribution of the tracer in the material, as well as the influence of microcracks on the oxygen diffusion. The results reported in references [20], [21], [22] are gathered on Figure 7c where Arrhenius plots of oxygen self-diffusion are drawn, corresponding to Arrhenius plots of volume and GB diffusion along the (ab) planes and parallel to the $\underline{\mathrm{c}}$ axis in single crystals. All these data are in general agreement with the oxygen diffusion behaviour in a ceramic, suggesting that oxygen diffusion in a polycrystal is clearly governed by GB diffusion. This latter is the limiting step, because the diffusion coefficient in the $\mathrm{CuO}_{2}$ superconducting (ab) planes is $10^{6}$ times larger than along the $\underline{\mathrm{c}}$ axis i.e. the direction perpendicular to the $(001)$ superconducting planes. This strong anisotropy of diffusion was observed and quantified for the first time on single crystals by Rothman at al [21] and on polycrystals by Routbort and Rothman [23].

An additional anisotropy of oxygen diffusion exists in the (ab) planes : the faster diffusion being along the $\underline{b}$ axis, i.e. along the $\mathrm{O}-\mathrm{Cu}-\mathrm{O}$ chains which carry the oxygen vacancies. Therefore, the anisotropic oxygen diffusion in the (001) planes and along the [001] direction of the lattice will strongly affect oxygen diffusion inside each grain of the ceramic during processing. Therefore, the sample geometry will strongly affect the final properties of the ceramics. This explains the smaller critical density values found in bulk samples, compared to thin films which are deposited according to a favourable orientation.

\subsection{Sintering parameters}

Generally, sintering requires a high temperature step for the growth, followed by cooling to room temperature. In these conditions, the (123) phase obtained is not superconducting and is generally oxygen deficient. It requires a second step of annealing around $823 \mathrm{~K}$ in order to optimize the oxygen content. Even if the initial powder is stoichiometric, the synthesized(123) phase contains oxygen vacancies. Consequently, there may be local changes in the microstructure and in properties which are related to the oxygen content, such as critical temperature, lattice parameters.

Various sintering parameters are exploited to densify the material, to reach the maximum oxygen content of the (123) orthorhombic phase and to produce various microstructures with specific features. The challenge in synthesizing dense materials is to avoid microcracks due to an inhomogeneous distribution of grain sizes and to keep an adequately interconnected 
porosity inside the material, which ensures oxygenation into the bulk.

Four main steps are involved in the growth of the (123) phase:

(i) The first one is the preparation of the starting powders from different materials: oxides, nitrates or carbonates. According to their nature and quality, various kinds of ceramics may be obtained. These initial powders must consist of a high purity single phase to ensure the formation of the single(123) phase as reported in reference [24].

(ii) The second step, sintering at high temperatures, requires well-defined experimental conditions concerning the heating rate, the annealing time for the grain growth and the oxygen gas flow. These conditions will determine either solid-solid or solidliquid diffusion reactions.

(iii) The third step is the cooling stage from high temperature, which is performed either with a low cooling rate or by quenching under oxygen gas flow, depending on the nature of the study. Slow cooling stabilizes the structure and morphology of the (123) phase. Quenching is the only way of preserving metastable high temperature phases.

(iv) The fourth step is the oxygenation of $\mathrm{YBCO}:$ it is performed by annealing between $753 \mathrm{~K}$ and $823 \mathrm{~K}$ under oxygen gas flow. This step, and particularly the choice of the annealing time at this temperature, will determine the superconducting properties.

As these parameters act simultaneously, all these steps are critical and have to be controlled, because they strongly influence the physical properties, microstructure and chemical composition of the synthesized ceramics.

Various types of sintering were investigated for the synthesis of bulk ceramics. Among them we find classical sintering [25], [17], hot-press sintering [26], [27], [18], [19], and melttextured sintering [28], [29], [30], [31]. For classical sintering, powders are pressed into pellets at room temperature under uniaxial or isostatic pressure, and then sintered. In hot-press sintering, an isostatic pressure from 5 Kbars to 40Kbars [18] is maintained during sintering. In the melt process, pellet sintering is performed with a thermal gradient applied to the substrate [30], [32], under a magnetic field [31] or using flux growth from a seed [33], [34]. Thermodynamic equilibrium and out-of-equilibrium states vary from one type of sintering to the other one and can also vary inside a given ceramic from one point to another. Considering all the types of sintering which have been investigated, it appears that hot-press processing and the melt-textured process are the best means to increase Jc. The former implies solidsolid and the latter solid-liquid diffusion. 


\section{Grain boundaries from solid-solid diffusion}

\subsection{Influence of processing on grain boundary microstructure and properties}

Studies by Ayache et al [25, 35] have reported the influence of sintering conditions on microstructure and physical properties. They described the effects of the high temperature annealing stages, of the oxygenation step, and those of either a new annealing using classical sintering, or of hot-press sintering under various conditions. Initially, two samples were hotpressed and sintered at $1199 \mathrm{~K}$ during respectively $13 \mathrm{~h}$ (sample $\mathrm{A}_{0}$ ) and $96 \mathrm{~h}$ (sample $\mathrm{B}_{0}$ ). Next, they were submitted to an oxygenation step at $753 \mathrm{~K}$ (respectively for $24 \mathrm{~h}$ and $72 \mathrm{~h}$ ). Several effects of the high temperature stage were observed: increase of the density from $88 \%$ to $92 \%$, of the grain size from $3 \mu \mathrm{m}$ to $5 \mu \mathrm{m}$, of the critical temperature Tc from $43 \mathrm{~K}$ to $63 \mathrm{~K}$, of $\mathrm{Jc}$ by a factor of 3 from 130 to $393 \mathrm{~A} / \mathrm{cm}^{2}$, and a decrease of the width $\Delta \mathrm{Tc}$ from $16 \mathrm{~K}$ to $12 \mathrm{~K}$. A second sintering (samples $\mathrm{A}_{1}$ and $\mathrm{B}_{1}$ ) favoured the increase of $\mathrm{Tc}$ and a decrease of $\Delta \mathrm{Tc}$, improves the magnetic property of the grains, i.e. the shielding efficiency was much better in the second sample inside the grain, although Jc became smaller. It was also shown, from a grain boundary study of YBCO sintered under quasi-isostatic pressure, that a small change in the oxygenation temperature during the final step produces a drastic change in the microstructure (density, grain size, oxygen content from $\mathrm{O}_{7}$ to $\mathrm{O}_{6}$ ) of different types of GB's (clean versus damaged GB) and in the superconducting properties.

\subsection{Influence of microstructure on transport properties}

The superconducting transition can be observed by plotting the resistance as a function of temperature. The transition profile can be separated in two regimes [36], [37], [38]. The first one, called the main transition regime, corresponds to the drop of the resistance in the grains. It depends mainly on the chemical composition and on the oxygen stoichiometry of these grains. The second one, lying immediately below the main one and covering the range down to zero resistance, is the tail regime. Transport in this regime is controlled by the grain boundaries (weak-links) and corresponds to the temperature range where the Josephson coupling energy of the weak-links is insufficient to overcome the thermal energy. This regime becomes broader with the presence of defects in the grain boundaries (intergranular phases, misorientations, GB oxygen deficiency, or GB cationic deficiency). Figure 8 shows the experimental $R(T)$ measurements obtained from sintered samples $A_{0}, A_{1}, B_{0}$ and $B_{1}$. The results show clearly an increase in the transition width as Tc decreases, revealing a low oxygen content and a low homogeneity in the ceramic, and also the presence of secondary phases at grain boundaries. The curves also indicate that an additional sintering of sample $\mathrm{A}_{0}$ and $\mathrm{B}_{0}$ improves the transition. The semi-conducting behaviour of sample $\mathrm{A}_{0}$ has disappeared 
in sample $A_{1}$ which still exhibits a wide $\Delta T c$ and a tail region. In sample $B_{1}$, the oxygen content is optimum, but the tail effect has increased compared to sample $\mathrm{B}_{0}$.

Such an additional annealing increases the oxygen content and improves the quality of the weak-links, as shown by TEM analysis of sample $A_{1}$ (figure 9). This figure shows a grain surrounded by four other grains and particularly by different clean GB's (fig. 9a) with various misorientation angles : a small angle GB $\left(4^{\circ}\right)$ characterized by (001) planes in the two grains (fig. 9b), a clean triple junction showing twinning inside the grains (fig. 9c) and a facetted HAGB exhibiting (001) planes in one grain (fig. 9d).

GB TEM analysis of sample $\mathrm{B}_{0}$ indicates the presence of second phases like $\mathrm{BaO}$ (and/or $\mathrm{CuO}$ ) in HAGB (fig. 10). The presence of a second phase is often an indication of a local liquid state in the ceramic [35]. Figure 10a shows the HAGB change from a symmetric facetted GB (fig. 10b) to an asymmetric GB (fig. 10c) in the vicinity of an intergranular defect (fig. 10a). Such a GB has a special orientation close to the coincident site lattice (CSL) $\sum 13$ GB and contains (001) $\mathrm{CuO}_{2}$ superconducting planes which are favourable in terms of current. Even with a high angle misorientation of $67^{\circ}-69^{\circ}$, the GB could act as a Josephson junction weak-link. As this GB contains a second phase, it is not superconducting.

For sample $B_{1}, R(T)$ has been seriously improved in the first part of its profile (fig. 8), but the second part of this curve, corresponding to the tail regime where the transport is controlled by grain boundariy weak-links, becomes broader due to the presence of intergranular phases and GB oxygen deficiency. TEM analysis of this sample shows strong changes of the morphology, structure and chemistry on different scales. Figure 11 illustrates the resulting grain size gradient observed by polarized optical microscopy, scanning electron microscopy (SEM) and TEM. This ceramic contains elongated crystals $5 \mu \mathrm{m}$ to $100 \mu \mathrm{m}$ in length. This elongated shape (fig. 11a and fig. 11b), generally found in these ceramics, is due to the fact that the direction of maximum grain growth is parallel to the (ab) planes (anisotropic direction). The presence of cavities or microcracks, whose size ranges between $10 \mu \mathrm{m}$ to $20 \mu \mathrm{m}$, is due to an inhomogeneous grain size distribution and to grain misorientations. The external part is well oxygenated as can be seen from the splitting of the 110 reflections in the electron diffraction pattern (right part of fig. 11c). The inner part is characterized by a higher density, a lower oxygen content and the absence of splitting of 110 reflections (right part of fig. 11d). The oxygen gradient clearly affects the lattice parameters deduced from the diffraction patterns and also the twinning morphology. In particular, the twins present in the external part (left part of fig . 11c) of the ceramic are fully relaxed and the lattice parameters correspond to an orthorhombic structure with an oxygen content close to $\mathrm{O}_{7}$. The twins of the inner part are ferroelastic-like (left part of fig. 11d), and are not completely relaxed by the 
phase transformation. The lattice parameters are those of a tetragonal (123) phase with an oxygen content close to $\mathrm{O}_{6.5}$. The microstructure of this inner part explains the remaining tail region in the $\mathrm{R}(\mathrm{T})$ curve of figure 8 .

\subsection{Magnetic measurements and weak-link behaviour}

The use of hysteresis loop measurements under increasing magnetic field (from 0-250 mT to $\mathrm{H}_{\text {appl }}>\mathrm{Hc} 2$ ) was investigated on ceramics by [39] in order to measure the current density. Jc was calculated from Bean's Model [40]. The Jc versus field behaviour was very similar to that described by a simple self-field limitation hypothesis [41]. These authors found a good agreement between $\mathrm{Jc}$ values deduced from this model and from transport current measurements. Magnetic hysteresis loops under low magnetic fields at $4.2 \mathrm{~K}$ are directly correlated to the GB weak-link behaviour. The higher the hysteresis area, the higher is the shielding efficiency of the weak-links [39]. The shielding effect improves after a new annealing revealing a better magnetic behaviour inside the grains. Figure 12 gathers the Jc results calculated from Bean's model for samples $A_{0}, A_{1}, B_{0}$ and $B_{1}$

\subsection{Critical current density and grain size dependence}

Bulk ceramics show a strong dependence of Jc on grain size [25], with an exponential decrease, comparable to that found in Jc curves as a function of misorientation in the case of bulk bicrystals and thin film bicrystals (see paragraph 8). Jc values, calculated from magnetic hysteresis loops measurements, as a function of grain size are related to grain boundary transport in ceramics. The smaller the grain size, the higher is Jc. In figure 12 the results of classical sintering (ceramic $C$ ) and hot-press sintering (ceramics $\mathrm{A}_{0}, \mathrm{~A}_{1}, \mathrm{~B}_{0}$ and $\mathrm{B}_{1}$ ) are compared. The highest value of Jc is obtained by classical sintering which gives the lowest porosity $(78 \%)$, the maximum oxygen content $\left(\mathrm{O}_{7}\right)$ and the minimum grain size $(2 \mu \mathrm{m})$. The smallest value corresponds to the largest grain size (from 5 to $100 \mu \mathrm{m}$ ) with an inhomogeneous distribution, a high density (96\%) and an oxygen gradient between the inner and external parts of the material. Compared to classical sintering, the hot-press process has the advantage of giving the highest density ceramics. The drawback is the limited oxygen diffusion due to higher compacity.

There is a competition between density, grain size and oxygen content. Oxygen diffusion is favoured by porosities which play a possible role as diffusion paths. High density ceramics will have more difficulty to reach the maximum oxygen content, as compared to low density ceramics which will be fully oxygenated. The presence of a grain size gradient, as observed in sample $\mathrm{B}_{1}$, prevents the connection between grains and makes the $\mathrm{Jc}$ value decrease strongly 
(figure 12).

\section{Grain boundaries from solid-liquid diffusion : Melt-textured ceramics}

Melt-textured processes allow the synthesis of YBCO ceramics with highly improved transport properties in the presence of a large magnetic field, due to aligned microstructures almost free of weak-link grain boundaries [42], [43]. Melt-processing involves the peritectic melting of the YBCO phase well above the peritectic temperature $(1288 \mathrm{~K}$ in air), yielding a solid phase $\mathrm{Y}_{2} \mathrm{BaCuO}_{5}$ plus a liquid phase (called «m1») having an approximate stoichiometry $\mathrm{Y} / \mathrm{Ba} / \mathrm{Cu}: 1 / 16 / 27$ [44]. On subsequent slow cooling through the peritectic temperature, directional solidification of YBCO occurs. It was demonstrated from TEM chemical analysis of melt-textured samples that the YBCO phase grows directly from an undercooled yttrium-bearing liquid and not from heterogeneous nucleation sites on $\mathrm{Y}_{2} \mathrm{BaCuO}_{5}$ crystals [45], [46]. The growth rate limiting factor is expected to be yttrium diffusion at the liquid-YBCO interface. The difference between the growth rate parallel to (001) superconducting planes and that along the perpendicular direction $\underline{c}$, induces a microstructure characterized by aligned platelets separated by gaps [47]. These growthinduced defects are filled with secondary phases which have solidified from the trapped liquid. The knowledge of the stable and metastable equilibrium phases at high temperatures, especially in the YBCO primary solidification region, has proved essential to determine the effective conditions for both single crystal growth and YBCO peritectic crystallization. Until now, the solid-state equilibrium, the melting temperatures, the extension of the primary crystallization fields of the solid phases and their dependence on oxygen pressure have been extensively studied [48], [49], [50], [51]. A review of the chemical principles of the preparation of metal-oxide superconductors can be found elsewhere [52].

\subsection{Influence of processing on microstructure}

The advantage of melt-textured ceramics is the large extent of a single crystallographic domain, comparable in size to a single crystal. Therefore, they can be used for direct measurements of transport properties. Compared to ceramics made by a solid-solid diffusion process, melt-textured ceramics contain far less GB's due to directional melt growth. They look-like quasi «single-crystals» which contain, in addition, residual gaps from the crystallization of the liquid. These gaps appear as lines in optical microscopy images. Their microstructure also contains $\mathrm{Y}_{2} \mathrm{BaCuO}_{5}$ (211) precipitates resulting from the peritectic transformation. 
In these materials, Jc is mainly due to the pinning energy of the vortices at the interfaces between precipitates, (211) and (123) phases, and at twin grain boundaries. The striking feature of these melt-textured ceramics is that a single grain can extend over the whole length of the sample, particularly when the melt process is performed under a magnetic field. Such conditions favour the alignment of the superconducting planes parallel to the applied field [31], so that the sample contains only one or two large domains of the order of a few millimeters (10-30mm), separated by low angle GB's. As an example of YBCO melt-grown under magnetic field, figure 13a shows an optical polarized image with two large domains exhibiting a small misorientation of about $10^{\circ}$. TEM images (fig.13b) show that twins crossing the growth defect are either coherent or incoherent. The growth defect corresponding to a gap filled by the liquid during (123) growth, keeps the same orientation in the sample, parallel to the (001) superconducting planes. The (123) phase also keeps the same orientation across the (211) precipitates.

Besides, the melt-process is also commonly used [32] on substrates like $\mathrm{MgO}$ single crystals or polycrystalline $\mathrm{Y}_{2} \mathrm{O}_{3}, \mathrm{Y}_{2} \mathrm{BaCuO}_{5}$ or $\mathrm{Al}_{2} \mathrm{O}_{3}$. In these cases, the growth of $\mathrm{YBCO}$ gives rise to a higher proportion of grain boundaries compared to the magnetic field process which takes place without any support. Figure 13c shows an optical micrograph of YBCO melt-textured on a $\mathrm{Y}_{2} \mathrm{O}_{3}$ polycrystalline substrate. In the centre of the image,one can observe four different domains with a large GB misorientation of $67^{\circ}$. Here again the single domain extends over a few millimeters. The TEM image [46] of figure 13d exhibits characteristic clean interfaces between two (211) precipitates and the (123) phase.

Twins are the main GB's present in these materials. Figure 14a shows a polarized light micrograph of a melt-textured ceramic grown on a $\mathrm{Y}_{2} \mathrm{O}_{3}$ substrate, exhibiting white bands as a result of the twinned microstructure. The light lines correspond to (110) and ( $\overline{1} 10)$ twin domains. The twin width distribution appears non-uniform. In its centre, a long $89^{\circ}$ tilt grain boundary corresponds to a HAGB with an angle departing by $1^{\circ}$ from $90^{\circ}$ because of the two variants of the orthorhombic (123) structure. Figure 14b shows a TEM micrograph of this [001] tilt grain boundary with evidence of facetting of the (100) and (110) planes. Such a GB contains areas with symmetrical $90^{\circ}$ tilt GB's and asymmetrical tilt GB's. The symmetrical one is not energetically favoured, which explains oscillations between these two structures.

\subsection{Influence of the substrate on microstructure and physical properties}

Melt-growth under a magnetic field is directly performed on a (123) bulk ceramic. Applying a magnetic field gives the best results for Jc. In the case of melt-growth, with or without a thermal gradient, the ceramic is lying on a substrate whose reactivity determines the final 
superconducting properties. Because melt-growth results from the crystallization of a liquid brought at a high temperature, any interaction of the liquid with the substrate during the formation of the (123) phase can decrease Jc or, in the worst conditions, can destroy the microstructures and properties. Thus, Jc of melt-textured ceramics will be maximum only if no secondary chemical interaction takes place with the substrate. If the chemical composition of the substrate belongs to the phase diagram $\left(\mathrm{Y}_{2} \mathrm{O}_{3}\right.$ or $\left.\mathrm{Y}_{2} \mathrm{BaCuO}_{5}\right)$, there will be no additional phases. In the case of $\mathrm{Al}_{2} \mathrm{O}_{3}$ or $\mathrm{MgO}$ subtrates, chemical reactions lead to secondary phases and damaged microstructures. In particular, the use of $\mathrm{MgO}$ single crystal leads to the formation of a non-superconducting YBCO carbonate phase which can be found at the (123)-(211) interfaces as was reported by Gotor et al [53]. The carbon contained in $\mathrm{MgO}$ substrates diffuses into the liquid and alters the fabrication process. Long time preannealing of $\mathrm{MgO}$ substrates at high temperatures can cure this effect.

Two YBCO ceramics were synthesized by the melt-growth method. The first one (D), was prepared on a $\mathrm{MgO}$ substrate using a thermal gradient for texturing [54]. The second one (E) was grown on a sintered polycrystalline $\mathrm{Y}_{2} \mathrm{O}_{3}$ substrate which reacts with part of the liquid stemming from the melt. It forms an orientated layer of the $\mathrm{Y}_{2} \mathrm{BaCuO}_{5}$ (211) phase, which acts as a seed for the growth of (123) [55]. In both cases, after the high temperature cycle, a low temperature sintering was carried out to obtain the superconducting (123) phase with the good oxygen content and Jc was directly measured (see Table 2). Despite the long annealing time used for oxygenation, sample (D) exhibits a low critical temperature and a large transition width. Such a $\Delta \mathrm{Tc}$ indicates a low oxygen content, so as the presence of oxygen non-stoichiometry and secondary phases. Susceptibility measurements on sample (D) reveal both intragranular and intergranular magnetic behaviours denoting the presence of defects at grain boundaries and/or the formation of secondary phases in the melt-textured ceramic. TEM imaging (fig. 15) displays damaged interfaces between (211) and (123) phases due to carbon impurity diffusion proceeding from the $\mathrm{MgO}$ substrate [55]. The magnetic behaviour of sample (E) is the same as that of a superconducting single crystal, in which only the intragranular properties are visible, indicating that (123) GB's and (123)-(211) interfaces are clean as can be seen from figure 13d. The magnetic behaviour indicates a good shielding effect for both samples (D) and (E), although the GB current transport is null in sample (D) and high in sample (E).

\subsection{Melt-textured process as a means of studying YBCO grain boundary bicrystals}

The use of seed melt-processing allows the fabrication of larger samples with domain lengths as large as $20-50 \mathrm{~mm}$. In a TEM study aimed at high magnetic field applications, Zhang [34] 
found a twist component which forms the facetted boundary segments, in addition to the well known symmetrical $90^{\circ}$ tilt domain boundaries, and to the asymmetrical $90^{\circ}$ tilt boundaries. These GB's are expected to improve current transport in these materials.

The melt-textured process has also been applied to the synthesis of GB bicrystals in order to study their properties for specific orientations. Among these studies, Field et al [56] extended their investigations to transport properties and magnetic resistance properties across highangle bicrystals using melt-textured $\mathrm{YBa}_{2} \mathrm{Cu}_{3} \mathrm{O}_{6+\mathrm{x}}$. More recently, characterization of natural and artificial low-angle boundaries in YBCO melt-textured samples were investigated by Delamare et al [57]. Results obtained from a study of controlled bicrystal misorientation, by use dual seeding using single crystals of $\mathrm{Nd}_{1+\mathbf{x}} \mathrm{Ba}_{2-\mathrm{x}} \mathrm{Cu}_{3} \mathrm{O}_{\mathbf{y}}$. Todt et al [58] have been reported, giving Jc values very comparable to those observed in thin film bicrystals.

\section{Grain boundaries in epitaxial thin films}

Since the synthesis of $\mathrm{YBa}_{2} \mathrm{Cu}_{3} \mathrm{O}_{7-\mathrm{d}}$ thin films, there has been a tremendous interest in epitaxial thin films for fundamental studies of the the pairing mechanism, using Josephson and tunnelling junctions [7], [59], [60] and for electronic applications. Due to the short coherence length of these materials, high quality tunnel and Josephson junctions require S/N/S or S/I/S interfaces that are very sharp on the atomic scale, and therefore a surface roughness close to the lattice parameters.

\subsection{Influence of processing on epitaxial growth}

Perfect control of the growth is necessary to guarantee a reliable correlation between physical properties and film structure, especially for a contribution to the understanding of the superconducting mechanism. In particular, it is essential to define the optimum conditions for growing a single phase (123) superconducting thin film orientated along a specific direction, without secondary phases and without any oxygen deficiency which would strongly affect the study of its intrinsic properties. YBCO film morphology and microstructure are strongly dependent on the sintering process. The optimization of the deposit and the understanding of the dependence of its orientation on temperature, are related to the growth mechanism and to the nature of the substrate [61].

The most usual growth process for HTSC films is pulsed laser deposition which corresponds to a high energy process favouring out-of-thermodynamic-equilibrium conditions. It is difficult to accurately control the microstructure in such a process. In particular, the formation of high roughness, of out-growths, which are secondary phases produced during the process, 
are very critical and can change from one film to another. For example, in YBCO heterostructures grown by this process, the layer roughness increases with increasing thickness [62], [63] can reach the value of $5 \mathrm{~nm}$ deteriorating the local properties. Nevertheless, a lot of studies on bicrystal-based Josephson junctions, or on interface Josephson junctions, produced optimized films for the study of electronic properties [64], [65], [66], [67] studied in YBCO films the effects of cooling time on the electrical properties of grain boundary Josephson junctions with $24^{\circ}$ and $36.8^{\circ}$ tilt misorientations. A particular study of the effect of sintering, under various oxygen atmospheres, on the weak-link behaviour of YBCO by [68] demonstrated an improvement of the properties of the junctions. In the case of thermal evaporation, a low energy process (in the $10 \mathrm{eV}$ range), the growth rate can be very low $\left(10^{-1}-10^{-2} \mathrm{~nm} / \mathrm{s}\right)$ which allows a longer time for surface diffusion. This leads to a growth close to the thermodynamic conditions with no or only very few out-growths, and to a surface roughness as low as $0.3 \mathrm{~nm}$ for some orientations [69].

YBCO films grown by reactive co-deposition on $\mathrm{SrTiO}_{3}$ substrates have been recently used to make in-situ planar tunnel junctions $\mathrm{Pb} / \mathrm{YBCO}$ of high electronic quality and reproducible properties [70]. This process is optimized for a very low deposition rate $(0.00625 \mathrm{~nm} / \mathrm{s})$ on a substrate heated at $1023 \mathrm{~K}$. These conditions yield films of high crystalline quality. X-ray diffraction rocking curves on the intense 005 reflection and atomic force microscopy (AFM) measurements detect a low mosaicity of the film with FWHM $\approx 0.26^{\circ}$ and a surface roughness as low as $3.5 \mathrm{~nm}$ on a $1 \mu \mathrm{m}^{2}$ scale. The junctions grown in these conditions exhibit good conduction properties $(\mathrm{Tc}=89 \mathrm{~K}, \Delta \mathrm{Tc}=1 \mathrm{~K}$ for $50 \mathrm{~nm}$ thickness $)$ [71]. Using the same process, the best conditions to grow YBCO on a $\mathrm{MgO}$ substrate are different due to 3D island growth, whereas on $\mathrm{SrTiO}_{3}$ substrate $2 \mathrm{D}$ growth takes place. The optimum temperature on $\mathrm{MgO}$ is 1003K. Far from the optimum conditions, the roughness of the film increases drastically within a $10 \mathrm{~K}$ range and out-growths form on both $\mathrm{SrTiO}_{3}$ and $\mathrm{MgO}$ substrates [69]. It is important to point out the difficulty of preparing a pure [001] thin film without forming also another orientation, because of the closeness of the interfacial energies. In particular, [100] precipitates are often present in [001] YBCO thin films. They introduce $90^{\circ}$ planar and vertical GB's which behave as weak-link GB's and decrease Jc.

The anisotropy of the coherence length makes it difficult to obtain high quality interface junctions with specific orientations such as [100] and [110]. As an example, the growth of a film with a single [110] orientation is extremely difficult to achieve without forming also the [103] orientation. Only the use of special X-ray experiments for controlling the different steps in the formation of (123), makes it possible to obtain a single orientation [100] or [110] [69]. 


\subsection{Influence of YBCO growth on film microstructure}

$\mathrm{A} \mathrm{SrTiO}_{3}$ single crystal (cubic structure, a=0.3905nm) is the usual substrate for obtaining HTSC films and devices because of its dielectric constant and good matching with YBCO. When YBCO is grown on STO, [001] and [100] zone axes can be formed, depending on thermal conditions. YBCO growth on $\mathrm{MgO}$ leads to the formation of misorientations in the films due to $3 \mathrm{D}$ island growth and also due to the fact that commercial $\mathrm{MgO}$ is not a true single crystal as it contains numerous sub-grain boundaries. Consequently, YBCO epitaxial growth leads to strong planar misorientations around the [001] axis. Those observed from various studies are: $35^{\circ}, 30.6^{\circ}$ and $62.7^{\circ}$ [71], [72], [73], [74]. These particular values correspond in fact to coincidence site lattices (CSL) at YBCO GB's. Marshall and Ramesh [75] studied the effect of synthesis conditions on film orientation for different substrates like STO, $\mathrm{LaAlO}_{3}, \mathrm{MgO}$ and $\mathrm{Y}-\mathrm{ZrO}_{2}$ (YSZ). They found that STO was the best concerning lattice mismatch, orientation and $\mathrm{Tc}$. $\mathrm{MgO}$ and $\mathrm{YSZ}$ were the poorest substrates. On $\mathrm{MgO}$ they observed $8^{\circ}, 18^{\circ}, 26^{\circ}$ and $45^{\circ}$ misorientations. Other studies of YBCO grown on a YSZ single crystal substrate showed also various misorientated grain boundaries in the thin film [77]. These authors found misorientations of small angle $\left(6.5^{\circ}\right)$, high angle $\left(29^{\circ}, 45^{\circ}\right)$ and also $90^{\circ} \mathrm{GB}$ 's which correspond to the (ab) plane twinning.

The morphology of YBCO thin films consists of growth islands with terraces of either spherical or square shape, facetted or not, depending on growth conditions. The resulting surface may be either flat (2D growth) or rough (3D growth). Figure 16 shows YBCO thin films grown by a co-evaporation process respectively on STO (a, b, and c) and on $\mathrm{MgO}$ substrates (d, e and f). Figures 16a and figure 16d show AFM images of growth islands. Figures 16b and Figure 16e show TEM planar view images of a large area with a single [001] orientation on a STO substrate, and various GB misorientations around the [001] direction on a $\mathrm{MgO}$ substrate. The $\mathrm{GB}$ rotation angles of $35^{\circ}, 30,6^{\circ}$ and $62,7^{\circ}$ about the [001] direction are visible by the rotation angles between the twins of the two grains. Both films exhibit $90^{\circ}$ grain boundaries corresponding to (001) plane twinning. The Moires of figures $16 \mathrm{c}$ and $16 \mathrm{f}$ correspond to the superposition of YBCO 100 and 010 reflections of the (123) phase with those of the substrates. For a $\mathrm{MgO}$ substrate there is only one kind of Moire, whereas for STO there are chevron-like Moire structures exhibiting various d-spacings. Those formed on $\mathrm{MgO}$ have a constant value, indicating full relaxation in the film. The morphology of YBCO films on STO reveals a residual strain in the vicinity of the substrate.

All YBCO thin films contain growth defects induced by the epitaxial growth, and others resulting from the YBCO structure itself. Stacking faults due to the local chemistry (double 
$\mathrm{CuO}$ planes are often formed), antiphase grain boundaries created by the presence of steps located either near the surface of the substrate or along the island terraces, are observed. An other type of defect, always observed in $\underline{c}$-axis films, is the formation of local a-axis nuclei or grains formed mainly at the interface with the out-growths [71]. Their growth always competes with that along the $\underline{\mathrm{c}}$-axis as described by Granozio et al [77]. It depends mainly on deposition temperature and rate.

The critical thickness of YBCO on STO ranges between 15 and 30nm, which corresponds to the limit of elastic deformation in a YBCO film. In this range, growth takes place by a 2D to $2 \mathrm{D}-3 \mathrm{D}$ growth mechanism. Above this range, the growth is $3 \mathrm{D}$ and the film is relaxed. Its parameters tend towards those of the bulk. 50nm thick films grown on STO are still partially relaxed, while those on $\mathrm{MgO}$ are completely relaxed. In the case of $\mathrm{MgO}$, growth takes place by a straightforward 3D mechanism. This difference comes from the large mismatch between YBCO and $\mathrm{MgO} \delta a_{f} / a_{s}=9.26 \%$ and $\delta a_{f} / a_{s}=7.84 \%$ ), as opposed to the small mismatch for STO $\left(\delta a_{f} / a_{s}=2.17 \%\right.$ and $\left.\delta a_{f} / a_{s}=0.64 \%\right)$. This explains the formation of a YBCO/STO coherent interface and of a YBCO/MgO semi-coherent interface relaxed by dislocations. Films on $\mathrm{MgO}$ present a granular behaviour of the $\mathrm{R}(\mathrm{T})$ curve [70] with a tail regime due to growth island GB's.

\subsection{Influence of the YBCO growth process on grain boundary structure: thin film bicrystals}

There is a feature common to all YBCO thin films epitaxially grown on bicrystal substrates STO, $\mathrm{MgO}$ or YSZ: the meandering of the grain boundary. Numerous studies were reported concerning YBCO thin film bicrystals grown on STO bicrystal substrates, with the aim of making bicrystal based Josephson junctions. As YBCO is epitaxially grown on $\mathrm{STO}, \mathrm{MgO}$, or YSZ GB bicrystal substrates, knowledge of the bicrystal substrate boundary structure is essential for a perfect control of the process and of the intrinsic characteristics of YBCO junctions.

TEM investigations of YBCO thin films grown on various STO bicrystals with misorientations of $24^{\circ}, 36^{\circ}, 53^{\circ}$ and $67^{\circ}$ were performed by various research teams. [78], [79], [80], [81], [82], [83] From these studies, it follows that the meandering behaviour observed does not depend on the misorientation of the bicrystal, but on the growth of the thin film. Even for small misorientations, thin film samples show GB meandering.

Investigations of GB meandering in YBCO bicrystal thin films grown on $\mathrm{MgO}$ and $\mathrm{YSZ}$ bicrystal substrates were reported by Miller et al [79] for various misorientations $\left(10^{\circ}, 24^{\circ}\right.$, 
$36.8^{\circ}, 45^{\circ}, 53.2^{\circ}$ and $67^{\circ}$ ) as a function of the growth process. These experiments point out the strong dependence of the meandering width upon the growth process. In a more detailed study [84] compared, for a $36.8^{\circ} \mathrm{GB}$, the magnitude and wavelength of the meander in offaxis magnetron sputtered thin films grown respectively at a high rate of $0.4 \mathrm{~nm} / \mathrm{mn}$ and at a low rate of $0.16 \mathrm{~nm} / \mathrm{min}$. They found that the meandering width decreases as the growth rate decreases. In another study of the influence of low energy (co-evaporation) versus high energy processing (laser ablation) [85], [86], showed, in a near $\sum 13 \mathrm{~GB}$, that meandering decreases strongly for lower growth rates. They also observed an increased facetting of the growth islands and consequently of the meandering. Alarco et al [81] studied microstructures and properties of artificial grain boundaries in epitaxial YBCO thin films grown on [001] tilt YSZ bicrystals. They observed by TEM that a $45^{\circ}$ [001] tilt GB also presents some wavyness. They also detected the presence of [100] and [110] orientations in their film. Microstructure and Josephson phenomenology of $45^{\circ}$ tilt and twist YBCO artificial GB's were investigated by Tafuri et al [83] who also detected GB meandering.

A detailed study of a $24^{\circ}$ misorientated [001] tilt GB grown on a STO bicrystal by laser ablation, combining TEM, high resolution electron imaging (HREM), AFM, low voltage SEM and energy dispersive X-ray spectroscopy (EDS) observations was done by Ayache et al [82]. They determined the 3D grain boundary topography from quantitative analysis of HREM Moire images of the overlapping zones, and deduced a GB growth model. In this study they characterized, on different scales, firstly the orientation of the bicrystal substrate and the surface morphology before deposition, and then theYBCO GB's after growth. Moire analysis determined, from the change in lattice parameters, a variation in the oxygen content along the grain boundary, as well as a variation by $4^{\circ}$ of the angle of the GB misorientation. Figure 17 a shows a TEM image of a YBCO $24^{\circ} \sum 13$ [001] tilt GB grown on a STO substrate, exhibiting meandering of the GB plane. The electron diffraction pattern is visible in the insert. Figure $17 \mathrm{~b}$ shows a HREM image of a portion of the overlapping zones corresponding to the superimposition of grain 1 on top of grain 2 (or vice versa) along the GB, on either side of the GB plane. One can notice the oscillation and facetting of the GB plane.

The way the GB plane oscillates on either side of the GB line is determined by the underlying substrate. It also meanders all along the GB, changing from a symmetrical to an asymmetrical character, depending on its location along the GB and also on the film thickness. Such a GB oscillation induces strong local changes of the current from one place to another.

From the strikingly converging results obtained by investigating YBCO growth on top of a bicrystal, it has become clear that, during film growth, the YBCO grain boundary deviates 
from the path defined by the boundary of the underlying substrate. YBCO GB meandering behaviour is related to the nucleation and growth mechanisms of the film. All studies indicate that the extent of the meandering may reach 500nm. These appear as general features which confirm the correlation between island-like growth of the film and magnitude of the GB deviation, as well as the relation between shape and GB faceting. As an example, figure 18 shows AFM images of YBCO thin film GB's grown on a $24^{\circ} \sum 13$ [001] tilt GB. Figure 18a corresponds to the AFM image of a thin film made by laser ablation and figure $18 \mathrm{~b}$ to the AFM image of a film made by co-evaporation using a low deposition rate. They differ by the morphology of the growth-islands which present either square-shaped terraces or blurred facetting. The lower the rate, the larger are the facetted islands. Such island shapes are in accordance with what is expected from the thermodynamic conditions of the deposition. Those presenting a clear facetting correspond to the lower deposition rate, i.e. the one allowing the maximum time for atomic diffusion. The results given in the literature confirm that it is the kinetic factor which governs the growth, and not the epitaxial growth nor GB interfacial energies. At the beginning, the epitaxial growth determines the orientation. As growth proceeds, the driving force for the YBCO grain boundary structure becomes the growth mechanism. Figure 19a schematizes the shape of terraces with the holes formed via island coalescence, and figure $19 \mathrm{~b}$ shows the model derived from a quantitative analysis of the grain boundary which exhibits clearly an oscillation of the GB plane in the thickness of the film. The extent of the meandering on either side of the GB can vary from $10 \mathrm{~nm}$ to $500 \mathrm{~nm}$ depending on the growth conditions. As a result, the growth mechanism alters the epitaxial relationships between the YBCO film bicrystal and STO or MgO bicrystals at the GB plane, where the growth terraces come into contact. This situation becomes conflictual and the growth fronts interpenetrate, leading to alternating domains of overlap. These results could well affect the interpretation of the experiments which have been recently carried out to test the symmetry of the superconducting order parameter, through Josephson coupling measurements on grain boundaries with special orientations [87], [88].

An interesting microstructural study on YBCO bicrystal films grown on $\mathrm{MgO}$ substrates by liquid phase epitaxy [89], concerned large single facetted GB's. Various misoriented $4^{\circ}, 8^{\circ}$, $12^{\circ}, 16^{\circ}, 24,36^{\circ}$ and $45^{\circ}$ [001] tilt GB's were analyzed on different scales. TEM observations showed single straight facetting over a length of 10-30 $\mu \mathrm{m}$. HREM studies showed that all GB's were facetted on the atomic scale, except the $24^{\circ}$ and $36^{\circ}$ ones, on the scale of a few nanometers. The experimental Jc $(\Theta)$ curve overlaps that observed in classical thin films (see Figure 28) [90]. 


\section{Grain boundary structure and properties}

\subsection{Grain boundary structure and misorientation}

A study of small angle [001] tilt GB's by Tsu et al [91] showed facetting, dislocation structures and heterogeneity on various scales. More recently, TEM-EELS studies of hole concentrations near strongly and weakly coupled GB's associated with YBCO flux-grown bicrystals [33], [92] were performed on small and large angle (001) tilt pre-oriented GB's with misorientations of $7^{\circ}, 12^{\circ}, 28^{\circ}$ and $31^{\circ}$. The key aspect in this work is that the electromagnetic properties and the coupling character of each of the bicrystals were explicitly determined. It was shown that small angle GB's are strongly coupled and that their structures consist in primary GB dislocations. A slight hole depletion was noted near the GB's. In addition, some high angle, mainly [001] tilt GB's are weakly coupled and some are resistive at $\mathrm{T}>4.2 \mathrm{~K}$. In the former case, the cation structural width is apparently narrow, although GB's can be broad, exhibiting severely depleted zones. The resistive GB's contain a thin coating layer of a second phase: $\mathrm{Cu}_{2} \mathrm{O}$. The chemical composition at small and high angles shows a slight $\mathrm{Cu}$ enrichment at the GB's, in good agreement with the characteristic copper nonstoichiometry found in flux-grown YBCO ceramics [54]. GB enrichment in $\mathrm{Cu}$ comes from the flux-growth process. The oxygen hole deficiency zone extends over 20 to $40 \mathrm{~nm}$ into each crystal.

A systematic study [93] of the structure of symmetric and asymmetric tilt GB's was carried out using geometrical modelling and particularly the coincidence site lattice (CSL) configuration of the (a-b) and (a-c) types. The first one corresponds to atomic planes containing either $a, b$ lattice parameters, or $b, c$ lattice parameters adjoining the boundary. The YBCO layered structure allows four different types of atomic planes to meet at the interface both for a-b and a-c type boundaries. These are $\mathrm{Cu}-\mathrm{vacancy}-\mathrm{Cu}, \mathrm{Ba}-\mathrm{O}-\mathrm{Ba}, \mathrm{Cu}-\mathrm{O}-\mathrm{Cu}$ and $\mathrm{Y}-\mathrm{Y}$ atomic planes. In this modelling, the fraction of the continuous superconducting planes and the charge associated with the boundary void configurations were determined as a function of the misorientation angle of the $a-b$ boundaries. The geometrical modelling was extended to describe the distortions, the unbalanced charge and the continuity of the $\mathrm{Cu}-\mathrm{O}$ planes in the asymmetric GB's, and disclinations as well. The critical current density obtained in terms of the depression of the order parameter, the width, and the coalesced region of the boundary were found to agree with experimental observations. Figure 20 shows the resulting models obtained for $\sum 37, \sum 13, \sum 5$ large angle grain boundaries (20a, 20b and 20c) with their coalesced configuration. One can point out the distortion of the lattice superconducting $a-b$ planes and the voids at the grain boundary. Another example (Fig 20d) corresponds to the (ac) type GB, showing continuity of the lattice planes a and c, with a mixture of two types of 
structural units at the GB. The continuity of the $\mathrm{Cu}-\mathrm{O}$ planes responsible for the superconducting current is found to depend on the atomic species occupying the common lattice positions of the two adjoining grains. It also determines the critical current density of the GB's. The distribution of the excess charge at the grain boundary is shown to be important in scattering the superconducting pairs. The necessity of neutrality also leads to cation and anion vacancies at the boundary, in turn leading to non-stoichiometry.

A HREM study of current HAGB's like $\sum 190^{\circ}$ grain boundaries by Jia and Urban [94] showed different types of atomic planes at the interface. Three models have been deduced for thin film (100)/(001) interfaces projected along the [010] direction. These models are shown in figure 21 where figure $21 \mathrm{a}$ is the HREM image and figure $21 \mathrm{~b}$ shows the three models of the grain boundary. In model a,: grain 1 shows a (001) $\mathrm{CuO}_{2}$ plane at the upper grain face, and grain 2 a (100) Y-Ba-O plane at the lower face. In model b, grain 1 shows a (001) Ba-O plane and grain 2 a (100) $\mathrm{Cu}-\mathrm{O}$ plane; in model c, grain 1 shows a (001) $\mathrm{CuO}$ plane and grain $2 \mathrm{a}$ (100) Ba-O-Y-O-Ba plane.

\subsection{Grain boundary misorientation and oxygen content}

TEM-EELS (electron energy loss spectroscopy) studies by Zhu et al $[95,96]$ on YBCO bulk ceramics prepared via solid-solid diffusion allowed the analysis of low and high angle GB's and the measurement of the oxygen stoichiometry of GB's using the oxygen K edge pre-peak. It was found that small angle $\left(\theta<10^{\circ}\right)$ and high angle GB's $\left(\theta>10^{\circ}\right)$ can be either fully oxygenated or oxygen deficient. These results, obtained from $20 \mathrm{~GB}$ characterizations and exploited using the coincident site lattice (CSL) model, show that all high angle $\sum$ GB's (from $\sum 3$ to $\sum 20$ ) present a departure from perfect orientation varying between $3.24^{\circ}$ and $10.90^{\circ}$. All high angle $\sum 3, \sum 9, \sum 10, \sum 13$ and $\sum 26$ boundaries studied by Zhu [97] present an oxygen deficiency near the GB's over a width of about $80 \mathrm{~nm}$ on either side of each crystal. Figure 22 shows the variation of oxygen content for high angle grain boundaries close to $\sum 10$, $\sum 13$ and $\sum 26$.

Studies of [001] tilt GB's in YBCO thin films by HREM, Z-contrast and STEM-EELS [98], [99] showed the important role of symmetry in YBCO high angle CSL $\sum 5$ and $\sum 17$ GB's. The oxygen content does not change in symmetrical GB's, as shown by the presence of the oxygen $\mathrm{K}$ pre-edge peak, which is absent in asymmetrical GB's. Figure 23 reproduces the profiles of the oxygen K pre-edge peak used to quantify the oxygen content in GB's. Figure 23a shows the pre-edge peak corresponding to the superconducting $\mathrm{O}_{7}$ phase and figure $21 \mathrm{~b}$ shows the oxygen deficiency peak corresponding to the $\mathrm{O}_{6}$ non-superconducting phase. 
Oxygen deficiency in asymmetrical GB's appears clearly in the spatial profile of the normalized oxygen pre-edge peak [99].

\subsection{Grain boundaries in bulk and transport properties}

It is well known that the limiting factor for current transport is linked to intergranular GB's which are weakly superconducting areas. In granular superconductors, the current flows through complex paths formed by a junction network connecting the grains. Earlier reviews suggested that the limitation of Jc in YBCO materials had to do with both the lack of effective pinning of magnetic flux lines and with the presence of weak-link boundaries across which the supercurrent is limited. Later, based on studies of thin film GB's exhibiting a dependence of Jc upon misorientation, various authors claimed that not all GB's were suitable for high Jc transport. Even later, some high angle boundaries were found to have a noticeable Jc transport capability [100], [101], [102].

It is difficult to obtain high Jc values with classical ceramics, because of the presence of GB's with random orientations. A large number of GB orientations are present in a classical ceramic from small $\left(4^{\circ}\right)$ to large angles $\left(90^{\circ}\right)$, as well as special coincident site lattice $\Sigma$ HAGB's. In grain-boundary studies on bulk ceramics [95], found that the coincident-site lattice model allows the characterization of a number of grain boundaries present in YBCO as near CSL sigmas : $\sum 13\left(22.6^{\circ}\right), \sum 17\left(28.7^{\circ}\right), \sum 5\left(36.8^{\circ}\right), \sum 29\left(45^{\circ}\right)$ and: $\sum 1\left(90^{\circ}\right)$, as [001] tilt grain boundaries. A study of GB geometry and energy, in relation with critical currents in YBCO, analyzed numerous data from the literature and showed that random GB's are not clean and cannot transport the supercurrent, while small angle and some high coincidence GB's are clean and can transport the current [103].

A study combining TEM analysis, electrical and magnetic measurements [25] quantified the influence of sintering conditions on current density. Firstly, the variation of the current density in bulk ceramics shows a strong dependence on grain size. The smaller the grain size is, the higher is Jc. A decrease in grain size leads to an increase in the number of junctions, thus making more percolative paths available for current flow [104]. Secondly, due to the Josephson penetration depth of the current in the junction, the effective surface for current transport is weaker for large junctions than for small ones [105]. Thirdly, microcracks can be formed due to the anisotropy of YBCO grain growth. Microstructural studies displayed microcracks in grains larger than $10 \mu \mathrm{m}$ [106], [20]. The effect of microcracks is to break possible pathways for the current, thus leading to a decay of Jc. The observed drop of Jc as the grain size increases (Figure 12) is a combination of the these three phenomena.

In-situ TEM electrical experiments were carried out for various ceramics [107]. These authors 
detected, on large angle near $\sum$ GB's, previously characterized by $\mathrm{I}(\mathrm{V})$ measurements, a low attenuation of the supercurrent for HAGB's $\sum 29$ and $\sum 7$. In particular, they measured across a single GB like $\sum 7$ a drop of the grain current $\mathrm{I}(\mathrm{V})$ by a factor of 3 . As shown by HREM, boundaries which do not contain (ab) planes display a small atomic disorder. These experiments are interesting, but cannot be extended to predict a current in the bulk, due to connections with adjacent GB's. Reliable electrical measurements on a single GB are only possible on perfectly pre-oriented GB's.

On bulk GB bicrystals obtained via a melt-process, several workers [56], [57], [58] managed to produce a large range of misorientations from $1^{\circ}$ to $45^{\circ}$, controlling the growth using a thermal gradient and controlling the planarity of the grain boundary bicrystal plane. In a series of [100] tilt grain boundaries, they found a difference in critical current density of nearly two orders of magnitude between small $\left(\theta<10^{\circ}\right)$ and high $\left(\theta>20^{\circ}\right)$ misorientation angles. Figure 24 shows the variation of the $\mathrm{GB}$ current density with its two regimes [58]. This $\mathrm{Jc}_{\mathrm{GB}}$ curve shows a decrease of $\mathrm{Jc}_{\mathrm{GB}}$ as a function of the misorientation angle, similar to that seen in figure 12 as a function of grain size. The difference lies in the order of magnitude of Jc : it is $10^{2}$ times higher in thin film bicrystals than in bulk ceramics. This is in agreement with the larger number of grain boundaries in ceramics. Compared to thin film bicrystal GB's, displaying various misorientations of the grain boundary plane (as described below), bulk bicrystal GB's have a linear and planar GB plane with a single orientation. Such a bulk GB allows a less ambiguous interpretation of the relationship between microtexture and transport properties.

\subsection{Grain boundary structure and current transport modelling}

The limiting factor for transport properties in GBs is the intergranular critical current density. In many materials $\mathrm{Jc}$ is suppressed at grain boundaries by phenomena such as interface charging and electronic band structure bending. Current transport mechanisms through lowangle grain boundaries were investigated [108] by calculation of the solutions of the Ginzburg-Landau equation, which account for the observed rapid decrease of the critical current Jc as a function of the misorientation angle. They proposed a mechanism of progressive superconductivity suppression in GB's as a function of $\theta$, due to an excess ion charge on the GB's which shifts the chemical potential in a layer of the order of the screening length in the vicinity of the GB's. They showed that the $\mathrm{Jc}(\theta)$ dependence is mostly determined by the decrease of the current-carrying cross section by insulating dislocation cores and by the progressive local suppression of the superconducting order parameter near 
the GB's as $\theta$ increases. The insulating region near the dislocation cores results from a local strain-induced transition to the insulating antiferromagnetic phase.

When the misorientation increases, the separation of the dislocation cores decreases. At the point where the strain fields around dislocation cores begin to overlap, i.e.at the end of the low angle regime (for large HAGB's), models based on linear elasticity become inapplicable. At this stage, the alternative methodology to describe the grain boundary structure is by structural units as shown by Browning at al [99]. The advantage of such a model is that, once the structural unit has been determined, it is possible to predict the structure of the GB for any orientation. But such a deduction in perovskite oxide GB's is very difficult due to the inhomogeneity along the atomic GB's structure. As an example, Ayache et al [109] found new atomic structural units of a symmetric [001] $67.4^{\circ}$ near- $\sum 13$ tilt grain boundary in $\mathrm{SrTiO}_{3}$ identified by a quantitative analysis of lattice images, reconstructed electron exit waves, and Z-contrast images. They found evidence for the formation of three new structural units centered either on $\mathrm{Sr}$ columns or on $\mathrm{TiO}$ columns. This GB presents a multiplicity of structural units (i.e. various stoichiometries along the GB) which contain 4 and 5 columns in coincidence, and the same number of doublets of columns of the same nature in close proximity. These results highlight new interpretations of the formation of grain boundary structural units in perovskite oxide system.

Atomic scale modelling of the GB potential in cubic perovskite oxides was developped by Klie et al [110], based on a combination of experiments on $8^{\circ}$ and $58^{\circ}$ [001] tilt $\mathrm{SrTiO}_{3}$ GB's and on theoretical calculations. Distance-valence least square analysis and multiple scattering calculations were used to determine the density of the GB states. They showed, for both high and low angle tilt GB's, the validity of the Thomas-Fermi approach of screened charges associated to the classical Schottky model. Figure 25 ( $a$ and $b$ ) shows the density of states for two $\mathrm{SrTiO}_{3}$ GB's (pristine). One can see clearly the charge distribution in the dislocation cores for low (a) and high (b) angle GB's. The smallest misorientation corresponds to the largest distance between the dislocations cores avoiding the overlap of the strain field [110]. In comparison, figure $25 \mathrm{c}$ shows the charge distribution in a $4^{\circ}$ [100] tilt boundary in Cadoped (left) and undoped (right) YBCO [111]. The core centre corresponds to a negative charge.

A numerical simulation study of the influence of temperature and interface space charge on the GB conductivity in $\mathrm{Ni}$ acceptor-doped $\mathrm{SrTiO}_{3}$ ceramics [112] showed that the electrical characteristics of these GB's could be deliberately modified by decorating the GB's with suitable dopants $(\mathrm{Ni}, \mathrm{Ag}, \mathrm{Ca})$. 


\subsection{Grain boundaries in thin films and transport properties}

The first studies of transport properties in thin film bicrystals were performed on samples prepared by laser ablation [113]. Various STO bicrystals with misorientation angles ranging from $0^{\circ}$ to $35^{\circ}$ were produced by melt-processing and used as substrates for growing YBCO bicrystals films in order to study the orientation dependence of the GB critical current . The critical current density across GB's on the (ab) basal plane of YBCO bicrystals was measured at $4.2 \mathrm{~K}$ as a function of misorientation. The ratio of grain boundary critical current density $\left(\mathrm{Jc}_{\mathrm{GB}}\right)$ to average value of the critical current density in the two grains $\left(\mathrm{Jc}_{\mathrm{GB}}\right)$ was plotted as a function of misorientation (Fig.26). The $\mathrm{Jc}_{\mathrm{GB}} / \mathrm{Jc}_{\mathrm{G}}$ ratio decreases exponentially with increasing GB misorientation .

This curve shows that, even for $0^{\circ} \mathrm{GB}$ misorientation, $\mathrm{Jc}_{\mathrm{GB}} / \mathrm{Jc}_{\mathrm{G}}$ decreases by a factor of two. This means that, even for a junction free from any misorientation, coupling between the two grains is decreased. Due to the sensitivity of Jc to crystallographic orientation, the coupling is maximum along the crystallographic axis and decreases with increasing GB misorientation. Such a behaviour cannot be related to classical coupling, but rather to pinning. Results obtained from the study of the residual critical current in HTSC bicrystal GB junctions tend to support such an hypothesis [65]. They showed that the residual current increases as the misorientation decreases. In this kind of systems, the residual critical current is attributed to inhomogeneities inside the barrier. The GB model proposed considers a periodic modulation of the order parameter, associated with a periodic structural change element which is consistent with only some of the properties of GB's junctions [114], and also with the presence of a residual current. Within the framework of such a model, defects such as dislocations depress the GB order parameter. A GB can be described as an array of a large number of parallel Dayem bridges [115] separated by normal areas. Figure 27 shows such a grain boundary model schematically.

Magneto-optical studies of flux penetration and critical current density in [001] tilt YBCO thin film bicrystals were made for small misorientation angles $\left(5^{\circ}<\theta<7^{\circ}\right)$ by Plyanskii et al [116]. They found a characteristic cusp in the flux distribution, which indicates that the critical current density $\mathrm{Jc}_{\mathrm{GB}}$ across the $\mathrm{GB}$ is smaller than the $\mathrm{Jc}_{\mathrm{G}}$ of the grain. They used Bean's model to separate the GB contribution from that of the grains. They found that small angle grain boundaries can provide additional flux pinning. This is plausibly associated with GB dislocations which accommodate the GB misorientation.

Another recent work on grain-boundary dissipation in HTSC's by Gray et al [90] reexamined the explanation of the zero field data obtained for thin films and bulk bicrystals 
[001] tilt GB's. Their $\mathrm{Jc}_{\mathrm{GB}}$ values are plotted in Figure 28 as a function of the misorientation angle. Both bulk and thin film bicrystals appear to behave in the same way. This work took advantage of microscopically more perfect bicrystals. The explanation is based on the pinning of Josephson junction vortices by the meandering found in thin film GB's. These authors also point out that there is no evidence that $\mathrm{Jc}_{\mathrm{GB}}$ of $\mathrm{GB}$ 's does not drop with applied magnetic field as quickly as expected from a simple Josephson junction model. Experiments [116], [65] and modelling [117] show that the pinning of vortices is a key process involved in the superconducting behaviour of GB's, as well as in the dissipation in the bulk. The Gurevich and Cooley mechanism implies that the trapped flux can improve the critical current Ic, if it is not governed only by grain boundaries.

\subsection{Critical current density and doping of the grain boundaries}

The critical current density Jc of the supercurrent which can pass through polycrystalline materials without destroying superconductivity is remarkably small. There have been various attempts to enhance the transport properties of multicrystalline ceramics and to understand the mechanism leading to a reduction of the critical currents at GB's. Appropriate doping of the grains provides a mean of optimizing the transport properties of GB's in HTSC. This is demonstrated in the exemplary case of GB's in bicrystalline Ca-and Co-doped YBCO films [118]. By Ca doping, the critical current density is strongly increased and the normal state resistivity significantly reduced, as compared to the values obtained for equivalent junctions in undoped films.

Hammerl et al [119], investigating YBCO polycrystals, found that the partial replacement of yttrium by doping $\mathrm{YBa}_{2} \mathrm{Cu}_{3} \mathrm{O}_{7-\mathrm{d}}$ with calcium increased grain boundary $\mathrm{Jc}$ values substantially, but only at temperatures lower than $77 \mathrm{~K}$. The resulting doped grain boundary suggests a viable approach for producing cost-effective superconducting power cables operating at liquid nitrogen temperature. Recently, Klie et al [111] reported image simulations and spectroscopy at the atomic scale, that demonstrate that in poly-crystalline YBCO, highly strained grain boundary regions contain excess $\mathrm{O}$ vacancies, which reduce the local hole concentration. Calculation of $\mathrm{Ca}$ and $\mathrm{O}$ vacancy formation energies were performed using density-functional theory in the local density approximation. They deduce for bulk YBCO that $\mathrm{Ca}$ impurities indeed substitute for $\mathrm{Y}$, but in GB regions under compression and tension they also replace $\mathrm{Ba}$ and $\mathrm{Cu}$, relieving strain and suppressing $\mathrm{O}$-vacancy formation. They also used atomic resolution Z-contrast imaging in the STEM to show the arrangement of the cation columns at the grain boundary, in correlation with EELS to probe the concentration of $\mathrm{Ca}$ and the local concentration of holes. As a example, figure 29 shows atomic resolution Z-contrast 
images of an undoped pristine $\left(\mathrm{SrTiO}_{3}\right) 4^{\circ}[001]$ tilt grain boundary and of a Ca-doped $4^{\circ}$ [001]tilt YBCO GB [111]. Figure 29a shows dislocation cores in the typical pentagonal arrangement of the GB structural unit. This latter corresponds to one type of structural units found in $\mathrm{SrTiO}_{3}$ and described by Duscher at al [120]. Figure 29b shows the Y/Ba column pentagonal arrangement in the Ca-doped GB, which encloses three columns, two on the $\mathrm{Cu}-\mathrm{O}$ and one on the Y/Ba sublattice. Figures 29c and 29d show the scheme of the core structure where EELS probe analysis was performed. Their profiles through positions 1-5 show a high $\mathrm{Ca}$ content and less O-deficiency in the doped dislocation cores.

Another study on the modification of transport properties in low angle GB's via calcium doping of YBCO textured thin films [121], showed an increase of the critical density $\left[\mathrm{Jc}_{\mathrm{GB}}(\theta)\right]$ by up to $40 \%$ and $100 \%$ for respectively the $4^{\circ}$ and $8^{\circ} \mathrm{GB}$ 's. Considering the Gurevich and Pashitskii model [108] for current transport at low angles, the improvement can be attributed to a reduction of the strain field and the localized charges, leading to a reduced built-in potential in the dislocation cores.

For $5^{\circ}$ [001] tilt GB's, Ca addition is clearly benefitial, particularly when the sample is oxygen-treated to further overdope the sample after growth, as demonstrated by Daniels et al [122]. They showed that Ca-doping decreases excess GB resistance and diminishes the gap between inter-and intra-grain density over a wide field and temperature range. Another study investigated transport properties on low angle YBCO GB's of undoped and doped thin film bicrystals [123] of $4^{\circ}, 8^{\circ}, 12^{\circ}, 16^{\circ}$ misorientation angles. They reported that Ca-doped YBCO bicrystal films, with $10 \%$ Ca substitution, showed no significant Jc enhancement at $77 \mathrm{~K}$ in zero field, but a strong decrease of Jc at higher magnetic fields due to a reduced irreversibility field at 77K. Ag-doped YBCO films showed increased critical current densities at higher fields and a slightly shifted transition from strong to weak behaviour with respect to undoped YBCO bicrystal films.

Recently, direct evidence for negative grain boundary potential in Ca-doped and undoped $\mathrm{YBa}_{2} \mathrm{Cu}_{3} \mathrm{O}_{7-\mathrm{d}}$ was obtained using electron holography in a transmission electron microscope [124]. They found a reduction of negative charge at grain boundary dislocations in Ca-doped YBCO, as compared to undoped YBCO, and particularly a significant decrease of the size of the perturbed region in the Ca-doped samples (see Figure 25). This appears to be the principal mechanism for the improved interfacial superconductivity.

Very recently, statistical thermodynamics and kinetics were applied to study the origin and evolution of space charge and band-bending effects at low angle [001] tilt GB's and the effects of $\mathrm{Ca}$ doping upon them [125]. Atomistic simulations, using shell models of interatomic forces, were used to calculate the energetics of various relevant point defects. 
They found that segregation of calcium is strongly determined by the strain field, and that the nature of the space charge is closely related to the oxygen content. Doping calcium at small angle YBCO tilt GB's leads to an increase of Jc through an increase of the hole content, which enhances negative potential regions due to the segregation of calcium, and helps to passivate disorder at the boundary.

\subsection{Grain boundaries: from the bulk to thin films}

Table 3 summarizes the various types of ceramics investigated in the field of YBCO superconducting grain boundaries. It indicates also the GB quality, i.e. either clean or degraded (oxygen deficiency or cationic stoichiometry change) for small and high angle GB's.

Figure 28 reproduces the results taken from the literature, concerning the variation of Jc as a function of the misorientation angle in YBCO [001] tilt bulk [58], [126] and thin film [127], [128], [129] grain boundary bicrystals . The black symbols correspond to $\mathrm{Jc}_{\mathrm{GB}}$ in thin films and the white ones to bulk bicrystals. Grain boundaries, both in bulk and in thin films, have been improved with high quality materials allowing reliable measurement of the physical properties. Comparison of GB's $\mathrm{Jc}_{\mathrm{GB}}$ in the bulk and in thin films indicates clearly a similar behaviour as a function of misorientation. The Jc values in films are larger by a factor of $10^{2}$ than those for a perfectly defined bulk GB's, even though they have, on the atomic scale, a perfect GB plane linearity. The reason for such a behaviour lies in the pinning of the vortices along the GB plane [90]. In thin films, it is the growth island configuration which induces the high current density. This latter has its origin in the high pinning energy due to the film microstructure, i.e. dislocations at the interfaces between growth islands (Figure 17). These defects have a suitable size, of the order of the coherence length, so as a suitable orientation for pinning vortices. Comparing an equal GB misorientation in a bulk bicrystal and in a thin film bicrystal, the additional $\mathrm{Jc}$ observed is only due to the film growth. Finally, $\mathrm{Jc}_{\mathbf{G B}}$ determined by the misorientation itself is the same for bulk and thin films. On this basis Mannhart et al [130] devised their experiment for the creation of magnetic flux by single grain boundaries in YBCO.

In thin films, the critical current path across a grain boundary varies with the local misorientation. It can be represented by a diagram (fig. 30) which shows the combination of symmetrical and asymmetrical facetting on either side of the GB plane. With reference to calculations based on the influence of $d_{x 2-y 2}$ symmetry in device applications of HTSC grain boundary junctions, Mannhart et al [130] modelled the GB current density as a function of the 


\section{Conclusion}

All the data reviewed give evidence that grain boundaries in YBCO materials cannot be understood without having available specific information about their local composition, structure, ionic and electronic state. They confirm that studies of bicrystals are crucial for HTSC development. As a general rule, the observations are consistent with the predominance of current fluxes in the (001) superconducting planes of HTSC materials. Consequently, some high angle GB's can transmit a reasonably high supercurrent, provided they correspond to a coincidence relationship between adjacent crystals and also provided their configuration is compatible with an easy propagation of Cooper pair electrons in the $\mathrm{CuO}_{2}(\mathrm{ab})$ planes. 
For small angle GB misorientations $\left(\theta<10^{\circ}\right)$, the pinning contribution has to be taken into account in the models, in addition to the $\mathrm{d}_{\mathrm{x} 2-\mathrm{y} 2}$ symmetry of the order parameter. These implications are contained in the weak-link behaviour for large angle grain boundaries. Experiments and modelling also show that the pinning of vortices is a basic mechanism which governs the superconducting behaviour of GB's, so as the dissipation in the bulk. Accordingly, $\mathrm{Jc}_{(\mathrm{GB})}$ is determined by the misorientation itself, thus being the same for the bulk and for thin films. The current density difference $\left(\mathrm{Jc}_{\mathrm{GB}}\right)$ film - $\left(\mathrm{Jc}_{\mathrm{GB}}\right)$ bulk is only due to the growth of the film and to the induced structure of the GB plane.

\section{Acknowledgements}

My very special thanks to Mr. Michel Gasgnier for his contribution throughout the time of our collaboration and especially right now for his positive and constructive criticisms while rereading the manuscript. My thanks go also to Bernard Lang for his kind and efficient help in correcting my English writing.

\section{References}

[1]. Wu, M. K., Ashburn, J. R., Torn, C. J., Hor, P. H., Meng, R. L., GaO, L, HuAng, Z. J., Wang. Y. Q and CHu. C. W., 1987, Phys. Rev. Lett., 58, 908.

[2]. Maeda, H., Tanaka, Y., Fukutomi, M. and Asana. T ., 1988, Jpn. J. Appl. Phys. Pt. 2, 27, L209.

[3]. SHENG, Z. Z and HermanN. A. M., 1988, Nature (London) 332, 55.

[4]. GROSS, R., 1993, In Interfaces in High-Tc superconducting systems, ed. SL. Shinde, DA Rudman, Springer-Verlag, New York., 176.

[5]. BARONNET, A and PAGANO, S., 1994, J. Supercond. 7, 2, 375.

[6]. Specht, E. D., Sparks, C. J. Dhere, A. G., Brynestad, J., Cavin, O. B., Kroeger, D. M. and OYE, H. A., 1988, Phys. Rev. B37, 7426.

[7]. Tsuei, C.C., Kirtley, J.R., Chi, C.C., Yu-Jahnes, L.S., Gupta, A., Shaw, T., Sun. J.Z. and KETCHEN, M.B., 1994, Phys. Rev. Lett., 73, 593.

[8]. SigRist, M. and RiCE, T. M., 1992, J. Phys. Soc. Japan, 61, 4283.

[9]. Alario-Franco, M. A., CAPOni, J. J., Chaillout, C. and Chenevas, J., 1987, Mat. Res. Bull. 22, 1685.

[10]. Alario-Franco, M. A., Caponi, J. J., Chaillout. C., Chenevas, J. and Marezio, M., 1988, Mater.Res. Soc. Proc. 99, 41.

[11]. VAn Tendoloo, G., ZANDBERgen, H. W. and AMELInCKX, S., 1987, Solid State Commun, 63, 603.

[12]. Chaillout, C., Alario-Franco, M. A., Caponi, J. J., Chenevas, J., Hodeau, J. L. and MAREZIO, M., 1987, Phys. Rev. B36, 7118. 
[13]. Beyers, R and Shaw, T. M., 1989, Solid State Physics, Vol 42, 135.

[14]. CEDER, G., 1994, Molecular Simulation, 12, 141.

[15]. MatiC, V.M., Milic, M. and LaZAROV, N.DJ., 2000, Physica C, 339, 27.

[16]. Ambrosch-Draxl, C., Korzhavyi, P. A. and Johansson, B., 2000, Physica C, 341-348, 1997. [17]. AYACHE, J., GASGNIER, M., BoIRE, P. and WiCKER, A., 1991, Journal of the Europeen Ceramic Society, Euro-Ceramics II, vol 3, Eds. G. Ziegler, H. Hausner, Deutsche Kremische Gesellschaft, 2215.

[18]. Boire, P, Ayache, J., Chateau. C. and Leger, J.M., 1992, J. Mat. Sci. Eng., B14, 169. [19]. AyAChe, J., Boire, P., Fert, A.R., BOZEC. X., NAN CHEN and RoutborD. J.L., 1992, Supercond. Sci. Technol. 5, 634.

[20]. SAbras, J., Dolin, C., Ayache, J., Monty, C., MAURy, R and Fert, A.R., 1990, IIB89, J. Phys., Suppl au n ${ }^{\circ}, \mathbf{5 1}, 1035$.

[21]. Rothman, S.J., Routbort, J.L and BAKER, J.E., 1989, Phys. Rev. B40, 8852 .

[22]. Tu, K. N., Yeh, N. C., PARK, S. I. and Tsei, C. C., 1989, Phys. Rev. B39, 419.

[23]. Routbort, J.L and Rothman, S.J., 1989, DIF 89 Aussois, NATO, ASI France.

[24]. Legros, C., Haut, C., Ponsonnet-Mora, L. and AyaChe, J., 1999, J. Eur. Ceram. Soc 19 (2) 165-173.

[25]. Ayache, J., SAbras, J., Gasgnier, M., Monty, C., Maury. R., Bozec, X., Fert. A.R, Redoulès, J.P., SNoeck, E. and Roucau, C., 1990, J. Less .Com. Met., 164 \& 165, 152.

[26]. PANDEy, R. K.,Gilbert, G.R., KiRK, W. P., Kobiela, P. S., CleARlField, A. and SQuattrito, P. J., 1988, J. Supercond., 1, 45.

[27]. Aslan, M., Jaeger, H., Schulze, K., Frisch, A. and Petrow, G., 1990, J. Am. Ceram. Soc. 73, 2,450 .

[28]. Alford, N. M., Button, T. W., Pollard, R. J. and McCartney, D. G., 1989, J. Appl. Phys., 66, 5930.

[29]. Ginn, P., Chen, W., Zhu. N., LAmagan, M. and Balchandrian, U., 1990, Appl. Phys. Lett., 57, 1455.

[30]. Ayache, J., Pellerin, N. and Odier, P., 1993, Europ. Cer. Soc, Proceeding 3nd ECR'S Madrid, Vol 2, 485.

[31]. BArbut, N., Ayache, J., Chateignier, D. Bourgault, D., TOURnier, R., Gotor, F.J., Germi, P. and Pernet, M., 1994, Physica C, 235-240, 348.

[32]. Pellerin, N., Odier. P., Simon. P. and Chateignier, D., 1994, Physica C, 222, 133.

[33]. BABCOCK, S.E. and LARBALESTIER, D.C., 1994, J. Phys. Chem. Solids, 55, 1125.

[34]. ZHANG, X. F., 1998, Supercond. Sci.Technol.11, 1391.

[35]. AYACHE, J. and GASGNIER, M. ,1989, Intern. Conf. Supercond "ICMAS 89", Paris, 145.

[36]. GOLDSCHMIDT, D., 1989, Phys. Rev. B39, 2372.

[37]. GoldSCHMIDT, D., 1989, Phys. Rev. B39, 9139.

[38]. Rosenblatt, J., Raboutou, A., Peyral, P and Lebeau, C., 1990, Rev. Phys. Appl . 25, 73. 
[39]. Maury, R., Fert, A. R., Redoulès, J. P., Ayache, J., SAbras, J. and Monty, C., 1990, Physica C, 167, 591.

[40]. CHEN, D. X., SANCHEZ, A., NOGUES, J. AND MUOZ, J. S. (1990), Phys. Rev. B 41, 9510. [41]. Dersch, H. and Blatter, G., 1988, Phys. Rev. B 38, 16, 11391.

[42]. MuraKAMI, M., 1992, Supercond. Sci. Technol. 5, 185.

[43]. SAlAMA, K. and LEE, D., 1994, Supercond. Sci. Technol. 7 , 177.

[44]. Gervais, M., Odier, P . and Couture, J. P., 1991, Mater. Sci. Eng. B 8, 287.

[45]. Ayache, J., Pellerin, N. and Odier, P., 1994, Supercond. Sci. Technol., 7655.

[46]. Ayache, J., Gotor, F.J., Pellerin, N. and Odier, P., 1997, J. Mater. Res., Vol 12, N² 338.

[47]. Goyal, A., AleXAnder, K. B., Kroeger, D. M., Funkenbush, P. D. and Burn, S. J., 1993, Physica C 210, 197.

[48]. KIM, J. S. and GASKELL, D. R., 1994, J. Am. Ceram. 77, 753.

[49]. Krabbes, G., Bieger, W., Wiesner, U., Ritschel, M. and Teresink, T., 1993, J. Sol. State. Chem. 103, 420.

[50]. LEE, B. J. and LEE, D. N., 1991, J. Am. Ceram. 74, 78.

[51]. WONG-NG, W. and CoOK, L. P., 1994, J. Am. Ceram. 77, 1883.

[52]. Tretyakov,D., Yu, D. and Goodilin, E. A., 2000, Russian Chemical Reviews. 69 (1) 1.

[53]. Gotor, F.J., Pellerin, N., Odier, P., CAZY, E., Bonnet, J.P., Fert, A. and AyACHe, J., 1995, Physica C 247252.

[54]. Odier, P., Gotor, F.J., Pellerin, N., Lobbo, R.P.S., Dembinski, K., Ayache, J. Noel, H., Potel, M., Chaminade, J.P. and Collin. G., 1998, Mat. Sci. Eng., B52 117.

[55]. Pellerin, N., Gotor, F.J., Odier, P., Ayache, J., Fert, A., CAZY, E. and Bonnet, J.P., 1994, Physica C 235-240, 381.

[56]. FIELD, M.B., CAI, X.Y., BABCOCK, S.E. and LARBALESTIER, D.C., 1993, IEEE Trans. of Appl. Super., 3, 1479.

[57]. Delamare, M.P., Walter, H., Bringmann, B., Leenders, A. and FreyhardT, H.C., 2000, Physica C, 329, 160.

[58]. Todt, V. R., Zhang, X. F., Miller, D. J., St. Louis Weber, M. and DraVID, V. P., 1996, Appl. Phys. Lett., 69, 24, 3746.

[59]. Dimos, D., Chaudhari, P. and Mannhart, J., 1990, Phys. Rev., B 41, 4038.

[60]. Chaudhari, P., Mannhart, J., Dimos, D., Touri, C.C., Chi, J., OPrysko, M.M. and SCHEUERMANN, M., 1988, Phys. Rev. Lett. 60, 1653.

[61]. Miletto Granozzo, F., SalluZzo, M. and Scotti di Uccio, U., 2000, Phys. Rev. B, 61, 756.

[62]. Ayache, J., Contour, J.P., Dolin, C., Drouet, M. and Revelosona, D., 1997, J. Alloys. Compounds., 251, 185.

[63]. Ayache, J., Contour, J.P., Chenu, C., Drouet, M., Durand, O and Magis, M., 1999, Interface Controlled Materials (M. Rühle and H. Gleiter Editors) Vol.9 , 249.

[64]. Huang, Y., Merkel, K . L., Moeckly, B. H. and Char, K., 1999, Physica C 314, 36. 
[65]. Sarnelli, E., Chaudhari, P. and Lacey, J., 1993, Appl. Phys. Lett., 62, n 7, 777.

[66]. Ogawa, T. , 1999, Physica C, 313, 305.

[67]. OGaWA, T. 1999, Physica C, 320, 135.

[68]. PAthaK, L.C., Mishra, S.K., DAs, S.K., Bhattacharya, D. and ChOPRA, K.L., 2001, Physica C, 351, 295.

[69]. Kim, S.J., GRISON, X., AYACHE, J., LesueUR, J. and LALU, F. 2003, J. Cryst. Growth. 249, issues $1-2,186$.

[70]. LeSUEUR, J., APRILI. M., GRISON, X., KIM, S. J. and AYACHE, J., 1998, proceedings of SPIE Congress, San Diego.

[71]. KIM, S. J., AYACHE, J., GRISON. X., LeSUeUR, J., APRILI, M. and LALU, F., 1998, proceedings of SPIE congress, San Diego.

[72]. StreifFer, S.K., Lairson, B.M., EOMS, C.B., Clemens, B.M., Bravman, J. C. and Geballe, T.H., 1991, Phys. Rev., B43, 16, 43.

[73]. RAMESh, R., Hwang, D.M., BARner, J.B., NAZAR, L., RAVI, T.S., InAm, A., DutTa, B., Wu, X.D. and VANKATESAN, T., 1990, J. Mat. Res., 5, n 4, 704.

[74]. Williams, E.J. and Stobbs, W.M., 1993, Physica C, 208, 96.

[75]. MARShall, A. F. AND RAMESH, R., 1994, in Interfaces in High-Tc superconducting systems. Editors. Shindé. S. L. and Rudman. D. A., 71.

[76]. TIETZ, L.A. and CARTER, C.B., 1991, Physica C, 182, 241.

[77]. GRANOZIO, F.M., SALluZZO, M., SCOTTI DI UCCIO, U., MAGGIO-APRILE, I. and FisCHER, O., 2000, Phys. Rev.B 61, 1, 756.

[78]. Traeholt, C., Wen, J.G., Zandbergen, H.W., Shen, Y. and HilgenKamP, J.W.M., 1994, Physica C, 230, 425.

[79]. MilleR, D.J., RoberTs, T.A., KANG, J.H., TAlvacchio, J., BuchHOZ, D.B. and ChANG, R.P.H., 1995, Appl. Phys. Lett., 66, 2561.

[80]. Seo J.W., Kabius B., DÄhne U., Scholen A. and Urban K.,1995, Physica C, 245, 25.

[81]. Alarco, J.A., Olsson, E., Ivanov, Z.G., Winkler, D., STEPANTSOv, E.A;, LebedeV, O.I., VAssiliev, A.L., TZALENChuK, A.YA. and Kiselev, N.A., 1995, Physica C, 247, 263.

[82]. Ayache, J., Thorel, A., Lesueur, J. and DAhmen, U., 1998, J. Appl. Phys., 84, 94921.

[83]. Tafuri, F., Miletto Granozio, F., Carillo, F., Di Chiara, A., Verbist, K. and Van Tendeloo, G., 1999, Phys. Rev. B 59, n 17, 11523.

[84]. ZHANG, X. F., MILLER, D. J. AND TALVACCHIO, J., 1996, J. Mater. Res., vol 11, 1, 2440.

[85]. PASSERIEUX, G ., 2002, Thesis janvier 2002, Orsay, France.

[86]. Ayache, J., PasserieuX, G., Lesueur, J. and Lartigue. KorineK, S., 2005, Physica C., to be published.

[87]. Tsei, C.C., Kirtley, J.R., RupP, M., Sun, J.Z., Gupta, A., Ketchen, B., Wang, C.A., ReN, Z.F., WANG, J.H. and BHUSHAN, M., 1996, Science, 271, 329.

[88]. LesueUr, J. ApriLi, M., Grison, X. AyAChe, J., Kim S. J. and ThOREL, A., 1999, EdP Sciences, 
J. Phys. IV, Pr4, 207.

[89]. Wen, J. G., TAKagi, T AND KoshizuKa, N., 2000, Supercond. Sci. Technol. 13, 820

[90]. Gray, K.E., Miller, D.J., Field, M.B., KIM, D.H. and BERGHUIS, P., 2000, Physica C, 341-348, 1397.

[91]. Tsu, I.F., WANG, J.L., KAISER, K.L. and BABCOCK, S.E. 1998, Physica C. 306, issues 3-4, 163.

[92]. BABCOCK, S.E., CAI, X.Y., LARBALESTIER, D.C., Shin, D.H., ZHANG, N., ZHANG, H., KaISER

D.L. and GAO Y., 1994, Physica C, 227, 183.

[93]. JAGANNADHAN K AND NARAYAN.J, 1991, Materials Science and Ingineering., B8, 201

[94]. JiA.C. L AND URBAN K., 1993, interface science, 1, 291

[95].ZHU, Y.Z., WANG, Z.L. and SuenaGa, M., 1993, Phil. Mag. A 67, n 1, 11.

[96].ZHU, Y.Z., CORCORAN,Y.L. and SuENAGA, M. 1993, Interface Science 1, 361.

[97]. ZHU, Y. Z.,1996, Materials Science Forum,vol 207-209, 377.

[98]. Browning, N.D., Chisholm, M.F. and PennyCoOK, S.J., 1993, Interface science 1, 309.

[99]. Browning, N.D., Buban, J.P., Nellist, P.D., Norton D.P., Chisholm, M.F. and PennYCoOK, S.J., 1998, Physica C, 294, 183.

[100]. Chan, S. W., Hwang, D. M and NaZar, L., 1989, J. Appl. Phys. 65. 4719.

[101]. CHAN, S. W., HWANG, D. M., RAMESH, R., SAMPERE, S. M., NAZAR, L. AND GERHRARDT, R and PRunA, P., 1989, High Temerature Superconductor Thin Films : Processing, Characterization and Applications. Edited by Stockbaur. R. AIP proceeding 200, New York.

[102]. BABCOCK, S.E., CAI, X.Y. and LARBALESTIER, D.C., 1990, Nature 347, 6289.

[103]. ChAn, S.W., 1996, Materials Science Forum, Vols 207-209, 79.

[104]. Larbalestier, D. C. et al, 1988, Physica C, 153-155 1580.

[105]. Senoussi, S. Oussena, M., Aguillon, C. and Tremblay, P.,1988, J. Phys., C8, 12, 492099.

[106]. Smith, D.S., Suasmoro, S., Gault, C., Caillaud, F. and Smith, A., 1990, Rev. Phys. Appl. 25, 90 .

[107]. Laval, J. Y., Drouet, M., Swiatnicki, W., Gradys, E., SchiffMacher, G., MonOt, I and DESGARDIN, G., 1996, Mat. Sci.Forum, Vols 207-209, 737.

[108]. GuREVICH, A. AND PASHITSKII, 1998, Phys. Rev. B57, 21, 13878.

[109]. Ayache, J., Kisielowski., C.,.KilaAs, R., PASSERIEUX, G. AND Lartigue-KorineK, S., (2005) Journ. of Mater. Sci. 403091.

[110]. Klie, R. F., Beleggia, M., Zhu, Y., Buban, J. P. And Browning, N.D., (2003), Phys. rev. B68, 214101.

[111]. Klie, R. F., Buban, J. P., Varela, M., Franceschetti, A., Jooss, C., Browning, N.D., PANTELIDES, S. T. AND PENNYCOOK, S. J., 2005, NATURE, 435, 475.

[112]. HagenbeCK, R. AND WaSER, R., (1998), Journ. of Appl. Phys., 83, 4, 2083.

[113]. Dimos, D., Chaudhari, P., Mannhart, J. and LeGoues, F.K., 1988, Phys. Rev. Lett., 61, $\mathrm{n}^{\circ} 2,219$. 
[114]. Chaudhari, P. 1991, Physica C 185-189, 292.

[115]. DAYEM, A. H. and WIEGAND, J. J., 1967, Phys. Rev. 155, 419.

[116]. Plyanskit, A.A., Gurevich, A., PAShitski, A.E., Heining, N.F., REDWInG, R.D., Nordman, J.E. and LARBALESTIER, D.C., 1996, Phys. Rev. B 53, 8687.

[117]. Gurevich, A. and CoOley, L. D., 1994, Phys. Rev. B50, 13563.

[118]. Mannhart, J., Bielefeldt, H., Goetz, B., HilgenKamp, H., SCHMEhl, A., Schneider, C. W. AND SCHULZ, R. R., 2000, Physica C 341-348, 1393.

[119]. HAmmerl, G., Schmehl, A., Schulz, R. R., GoetZ, B., BielefEldT, H., SCHNEIDER, C. W., HilgenKAMP, H. AND MANNHART, J., (2000), Nature 407, 162.

[120]. Duscher, G. BubAn, J.P. Browning, N.D. Chisholm, M.F. PENNYCOOK, S.J., 2000, Interface Science, 8, 199.

[121]. Guth, H., Krebs, H. U., Freyhardt, H. C. And Jooss, CH., ( 2001), Phys. Rev. B64, 140508. [122]. Daniels, G. A., Gurevich, A. And Larbalestier, D. C., (2000), Appl. Phys. Lett. 77, 20, 3251.

[123]. Holzapfel, B., Verebelyo, D., Cantoni, C., Paranthaman, M., Sales, B., Feenstra, D.

C. AND Norton, D. P., 2000, Physica C 341-348, 1431.

[124]. SCHOFIELD, M. A., BELEGGIA, M., ZHU, Y., GUTH, K. AND JOOSS, C., 2004, Phys. Rev. Lett. 92, 19, 195502.

[125]. SU, H AND WeLCH, D. O., 2005, Supercond. Sci. Technol., 1824.

[126]. Field, M.B., Larbalestier, D.C., PARIKH, A. S. and SAlama, K., 1997, Physica C280, 221. and FIELD, M.B.(Thesis).

[127]. Ivanov, Z. G., Nilsson, P.A., Winkler, D., Alarco, J.A., Cleason, T., StePantsov, E.A. and TZalenchuK, A.Y., 1991, Appl. Phys. Lett. 59, 3030.

[128]. Heinig, N. F., REDWING, R. D., Tsu, I. F., GUREVICH, A., NORDMAN, J. E., BABCOCK, S. E., and Larbalestier, D. C., 1996, APPl. Phys. LetT., 69, 577 and Heinig, N. F. (Thesis).

[129]. Steel, D. G., Hettinger, J. D., Miller, D. J., YuAN, F., GRAY, K. E., KANG, J. H. AND Talvacchio, J., 1996, Appl. Phys. Lett., 68, 120.

[130]. HilgenKamp H., Mannhart J. and Mayer, B.,1996, Phys. Rev.B 53, 21, 14586.

[131]. BABCOCK, S.E. and VARGAS, J.L., 1995. Annu. Rev. Mater. Sci., 25, 193. 


\section{Figure Captions}

Figure 1: The various types of superconducting ceramics synthesized during the past decade with their respective Jc values: classical bulk ceramics, melt-textured bulk ceramics, bulk bicrystals, thin film bicrystals, thin films and single crystals. Note that the three bottom configurations are governed by controlled orientations.

Figure 2: Representation of a S/I/S junction with its characteristics for the Low Tc and High Tc superconductivity mechanisms.

Figure 3: (a) Structural unit-cell of the (123) phase showing $\mathrm{CuO}_{2}$ planes and $\mathrm{Cu}-\mathrm{O}$ chains. (b) Based on the $d_{x 2-y 2}$ symmetry of the superconducting order parameter, a schematic diagram of the strong coupling along [100] and [103] orientations, as opposed to the weak coupling along [001] and [110]

Figure 4: (a) Tetragonal and orthorhombic unit-cells of the (123) phase. (b) Evolution of the lattice parameter as a function of the oxygen deficiency d for various oxygen partial pressures. Note the change in the transition for temperatures between $798 \mathrm{~K}$ and $943 \mathrm{~K}$ [6].

Figure 5: Ternary phase diagram of the $\mathrm{BaO}-\mathrm{Y}_{2} \mathrm{O}_{3}-\mathrm{CuO}$ system showing the various possible secondary phases (132), (211).

Figure 6 : Structural phase diagram of the (123) phases displaying the effect of oxygen partial pressure and of temperature on the formation of the (123) oxygen stoichiometric phase [6].

Figure 7 : (7a) Oxygen diffusion depth profiling on a ceramic polished with a small angle $\left(\theta=1.56^{\circ}, \mathrm{T}=823 \mathrm{~K}, \mathrm{pO}_{2}=22 \mathrm{kPa}\right)$ exhibiting the presence of microcracks.

(7b) Ionic image obtained with $\mathrm{O}^{18}$ showing the anisotropy of oxygen diffusion.

(7c) Arrhenius plots of volume and grain boundary diffusion in ceramics along (ab) planes and parallel to the $\underline{\mathrm{c}}$ axis in a single crystal. Full triangles up and down represent respectively the coefficient of volume diffusion in the $\underline{c}$ direction and in (ab) planes [21].Diamonds represent the diffusion coefficient in polycrystals [23]. Squares and stars represent respectively volume diffusion and grain boundary diffusion [20]. Crosses represent the chemical diffusion coefficient taken from [22]. 
Figure 11 : Optical micrographs (a), SEM (b), TEM image and electron diffraction patterns (c and d) for ceramic B1, exhibiting morphologies resulting from the grain size gradient produced by the sintering. Note that twins formed in the external part of the ceramic are completely relaxed (left part of figure c) and have an oxygen content close to $\mathrm{O}_{7}$ as measured by the splitting of the 110 reflections (insert in diffraction pattern (c)). Twins of the inner part of the ceramic are of the ferroelastic type (left part of figure d). They are not completely relaxed by the phase transformation as deduced from the absence of splitting of the 110 reflections (right part of figure d) and their oxygen content is close to $\mathrm{O}_{6.5}$.

Figure 12: Critical current density Jc measured as a function of the grain size in bulk ceramics. $A_{0}, A_{1} B_{0}$ and $B_{1}$ samples correspond to hot-press sintering and sample $(C)$ to a low density material made by classical sintering.

Figure 13 (a) Polarized optical image showing two large domains with a low misorientation of about $10^{\circ}$, observed in a YBCO melt-textured ceramic synthesized under magnetic field. (b) : TEM micrograph of (a) exhibiting coherent and incoherent twins crossing a line defect. This growth defect, filled with liquid during (123) growth, keeps the same orientation, parallel to the (001) superconducting planes. (c) : Optical micrograph of a YBCO melt-textured ceramic grown on a $\mathrm{Y}_{2} \mathrm{O}_{3}$ polycrystalline substrate. This image shows four different domains 
with a large GB misorientation in its centre. Characteristic clean interfaces are formed between two (211) precipitates and (123) phases as seen in figure d.

Figure 14 : (a) Polarized light micrograph of a melt-textured ceramic grown on a $\mathrm{Y}_{2} \mathrm{O}_{3}$ substrate exhibiting white bands in the ceramic microstructure, and a $90^{\circ} \mathrm{HAGB}$ in its centre. Light lines correspond to (110) and (-110) twin planes. A non-uniform distribution of the twin widths can be observed. (b) : TEM micrograph of the same GB showing facetting along (100) and (110) planes. There are regions with $90^{\circ}$ tilt symmetrical and asymmetrical GB's.

Figure 15 : TEM image of a (123) phase / (211) precipitate interface damaged due to the presence of carbon impurities in the $\mathrm{MgO}$ substrate and due to carbon diffusion in the liquid.

Figure 16: YBCO Thin films grown by co-evaporation respectively on $\operatorname{SrTiO}_{3}(a, b$, and c) and on a $\mathrm{MgO}$ substrate ( $\mathrm{d}$, e and f). (a and d) AFM images showing the growth islands. (b) and (e) : TEM planar view image which shows large areas of the same [001] orientation in films on STO, and various GB misorientations around the [001] direction with rotation angles of $35^{\circ}, 30,6^{\circ}$ and 62,7 (visible by the twin rotation) for $\mathrm{MgO}$. Both films exhibit $90^{\circ} \mathrm{GB}$ 's corresponding to twinning of the (ab) superconducting planes. (e ) and (f) : Moires of the 100 and 010 reflections of the (123) phase with the substrates. One can notice that those present on STO (c) show a residual strain in the (123) film appearing in chevron- shaped Moires with various d-spacings, while those formed on $\mathrm{MgO}$ keep a constant value, indicating full relaxation of the film.

Figure 17 : (a) TEM image of a YBCO $24^{\circ} \sum 13$ [001] tilt GB grown on a STO substrate, exhibiting meandering of the GB plane. The electron diffraction pattern is visible in the insert. (b) : HREM image of part of the overlapping zones corresponding to the superposition of grain 1 on top of grain 2, or vice versa, along the YBCO GB. Note the facetting.

Figure 18 : AFM image of the same YBCO $24^{\circ} \sum 13$ [001] tilt GB. (a) corresponds to a laser ablated thin film of $120 \mathrm{~nm}$ thickness ; (b) co-evaporated thin film of 50nm thickness, grown using a low deposition rate. The morphology of the growth-islands presents either square shaped growth terraces or not very clear facetting. Notice a $20 \mathrm{~nm}$ wide area with a weak contrast of the island shapes, corresponding to the region of overlapping. 
Figure 23 : (a) EELS profile of the oxygen $\mathrm{K}$ pre-edge peak corresponding to the superconducting $\mathrm{O}_{7}$ phase; (b): oxygen peak deficiency corresponding to the $\mathrm{O}_{6}$ nonsuperconducting phase. (c) : Quantitative analysis of the normalized oxygen pre-peak showing oxygen deficiency in an asymmetrical GB (stars represent symmetrical and circles asymmetrical GB's) [100].

Figure 24 : Variation of the current density $\mathrm{Jc}_{\mathrm{GB}}$ of grain boundaries as a function of the misorientation angle between $1^{\circ}$ and $45^{\circ}$ in YBCO melt-grown bulk bicrystals [58].

Figure 25: (a and b) Density of states for these two $\mathrm{SrTiO}_{3}$ GB's (pristine). Notice the charge distribution in the dislocation cores for the low (a) and high (b) angle GB's. The smallest misorientation corresponds to the largest distance between the dislocations cores avoiding the overlap of the strain field [110]. (c) Charge distribution in a $4^{\circ}$ [100] tilt boundary in Ca-doped (left) and undoped (right) YBCO [123].

Figure 26 : Variation of current density Jc as a function of GB misorientation in YBCO thin 
film bicrystals [113].

Figure 27: Representation of a superconducting GB as an array of a large number of parallel Dayem bridges simulating superconducting regions (B) separated by normal or nonsuperconducting regions $(\mathrm{A})$.

Figure 28 : Variation of current density Jc of bulk and thin film bicrystals as a function of GB misorientation [91].

Figure 29 : Atomic resolution Z-contrast images of a pristine $4^{\circ}$ [001] tilt grain boundary in undoped and Ca-doped YBCO. (a) dislocation core with the typical pentagonal arrangement of structural units found in $\mathrm{SrTiO}_{3}$. (b) Ca-doped YBCO GB where the Y/Ba pentagonal column arrangement encloses three columns, two on the $\mathrm{Cu}-\mathrm{O}$ and one on the $\mathrm{Y} / \mathrm{Ba}$ sublattice. (c and d) diagram of the core structure where EELS analysis was performed [111].

Figure 30 : Representation of grain boundaries found in thin films containing a combination of symmetrically and asymmetrically facetted GB's. The critical current Ic across the grain boundary changes locally between null and a maximum value depending on the local crystallographic orientation of the GB faceting.

Figure 31 : (a) Model of the various GB geometries used for calculation; (b) Results from calculations by Mannhart et al [130] compared with experimental data [113]. 
1

2

3

4

5

6

7

8

Table 2 : Superconducting values for melt-textured YBCO ceramics prepared with a thermal gradient on a $\mathrm{MgO}$ substrate single crystal (sample D) and on a polycrystalline $\mathrm{Y}_{2} \mathrm{O}_{3}$ substrate (sample E).

Table 3 : GB's relative quality and Jc values for YBCO bulk, thin films, single and bicrystals. 


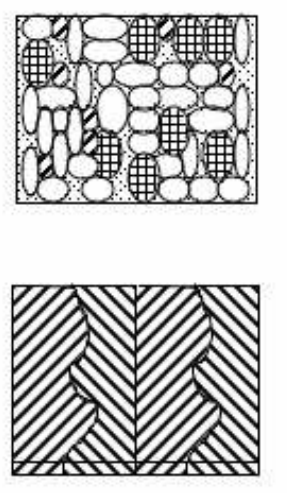

\author{
Classical \\ ceramics \\ Solid-solid \\ I $<<10^{3} \mathrm{~A} / \mathrm{cm}^{2}$ \\ Diffusion
}

Polverustals

Melt textured ceramics

$10^{4}<\mathrm{I} \mathbf{c}<10^{5} \mathrm{~A} / \mathrm{cm}^{2}$

Solid-liquid

On polvcristalline substrates

Diffusion

$\mathrm{Y}_{2} \mathrm{BaCuO}_{5}, \mathrm{Y}_{2} \mathrm{O}_{3}, \mathrm{MgO}, \mathrm{LaAlO}$

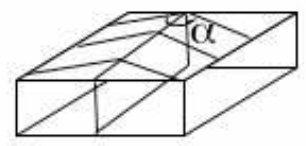

Bulk bicrystals

$\mathrm{Is}=\mathbf{f}(\boldsymbol{\alpha})$

Solid-liguid

From YBCO Melt ceramics

Diffusion

Thin film bicrystals

Is $=\mathbf{f}(\boldsymbol{\alpha})$

Solid-gas

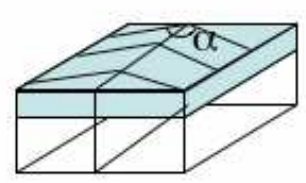

Grown on biccrystal substrates:

reaction

$\mathrm{SrTiO}_{3}, \mathrm{MgO}, \mathrm{Y}-\mathrm{ZrO}{ }_{2}$

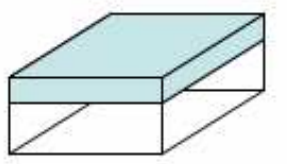

Thin films

$10^{6}<\mathrm{Ic}<10^{7} \mathrm{~A} / \mathrm{cm}^{2}$

\title{
Solid-gas
}

reaction

On single crystal substrates:

$\mathrm{SrTiO}_{3}, \mathrm{MgO}, \mathrm{LaAlO}_{3}$

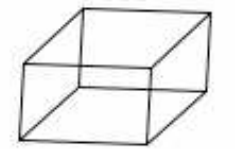

Single crystals

Solid-liguid

$10^{4}<\mathrm{I} c<10^{5} \mathrm{~A} / \mathrm{cm}^{2}$

Diffusion

Flux growth

\section{Figure 1}


Low Tc
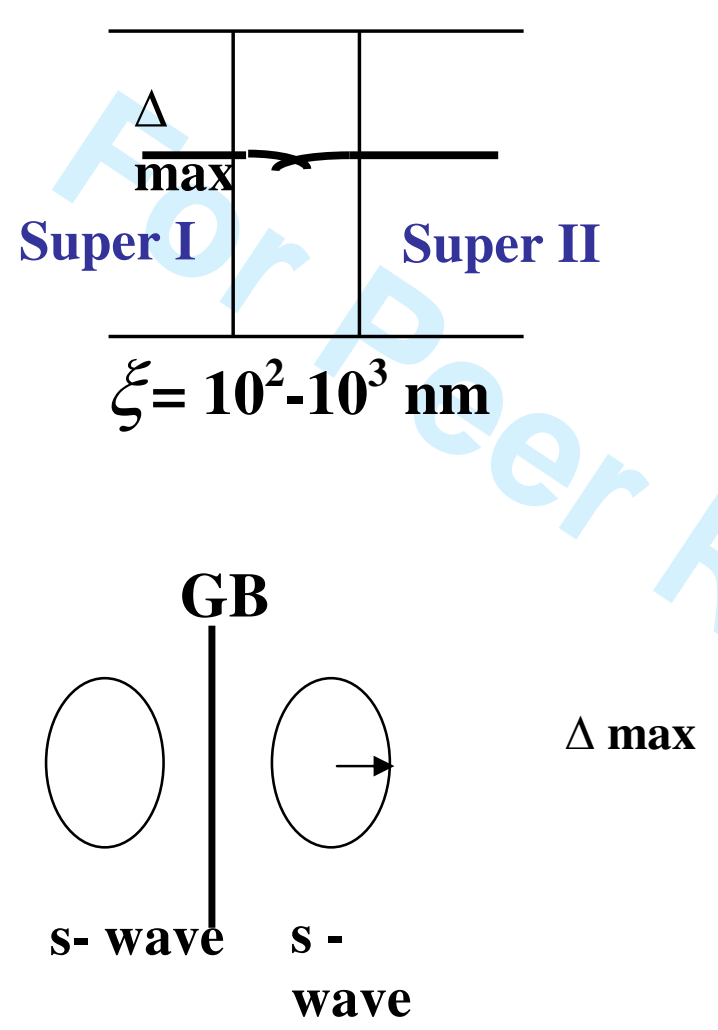

High Te

[010]

Figure 2 


\section{Figure 3}

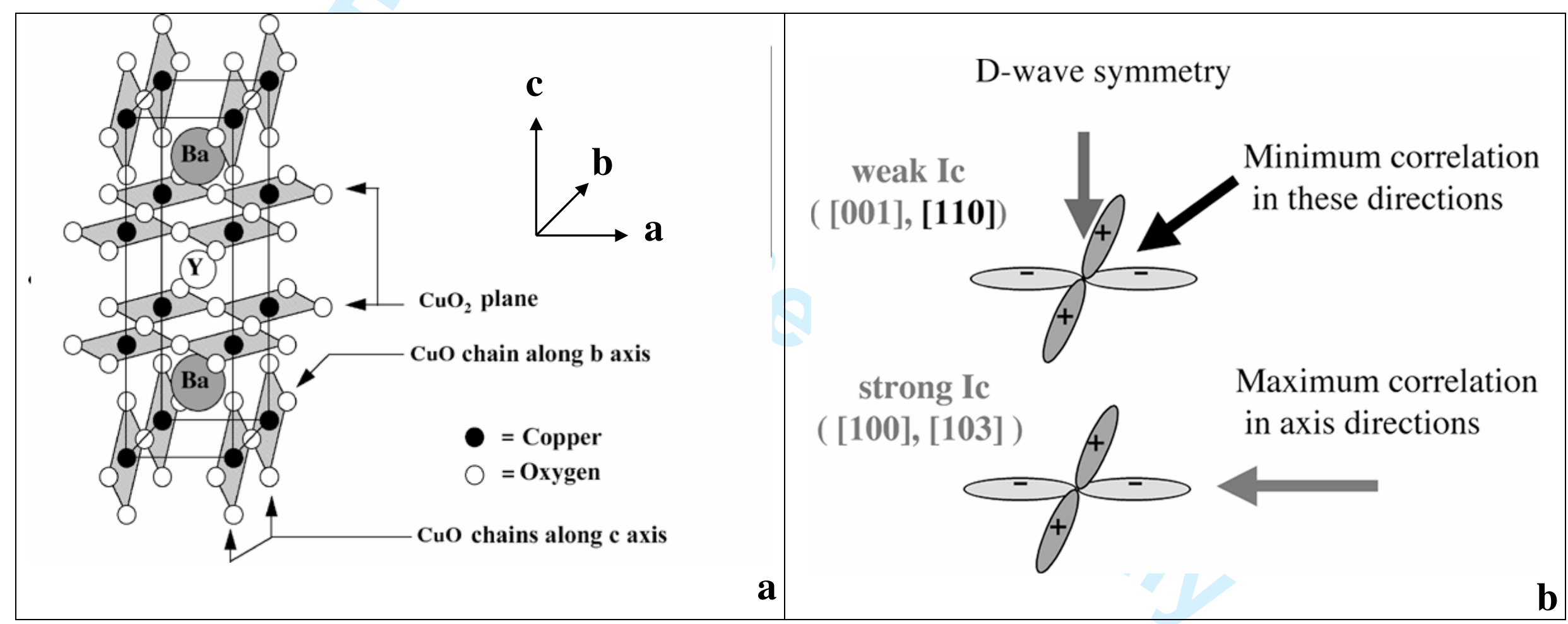




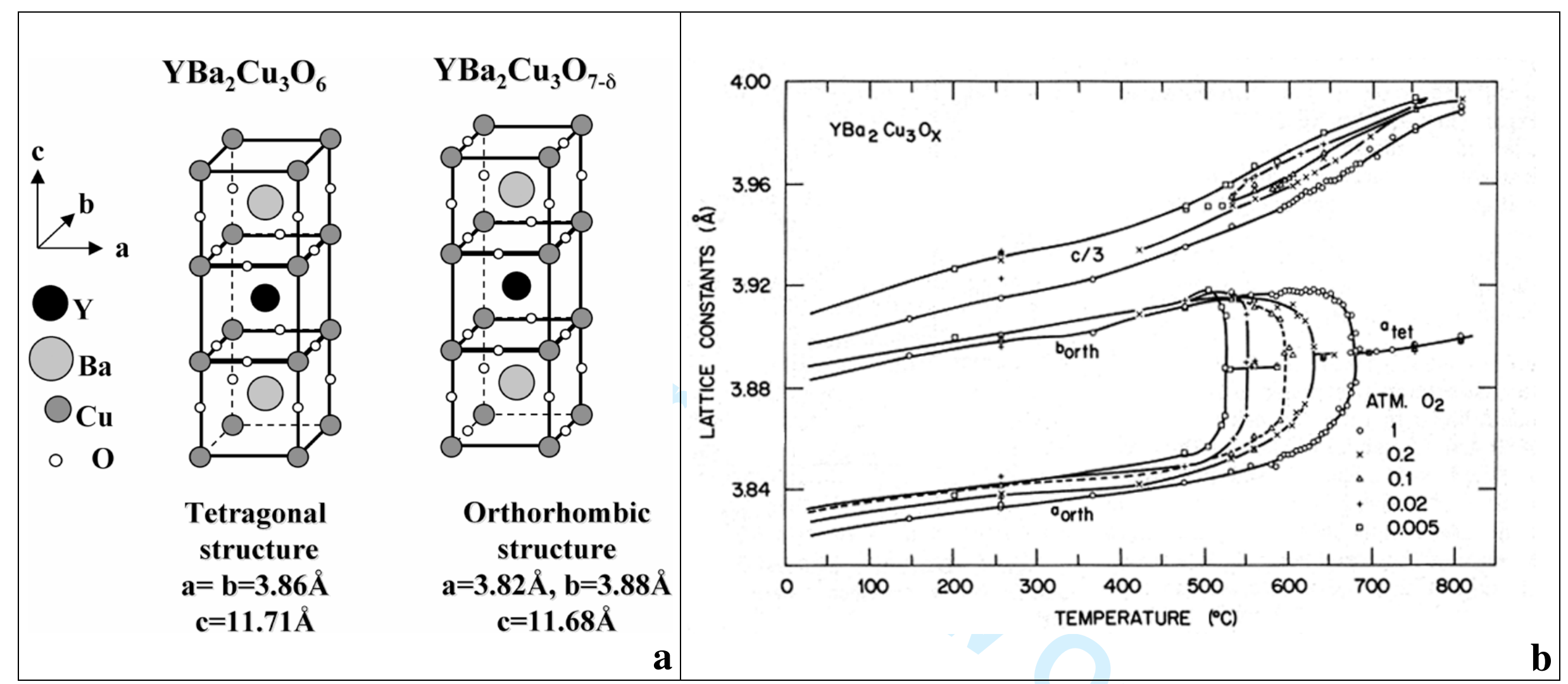

Figure 4 


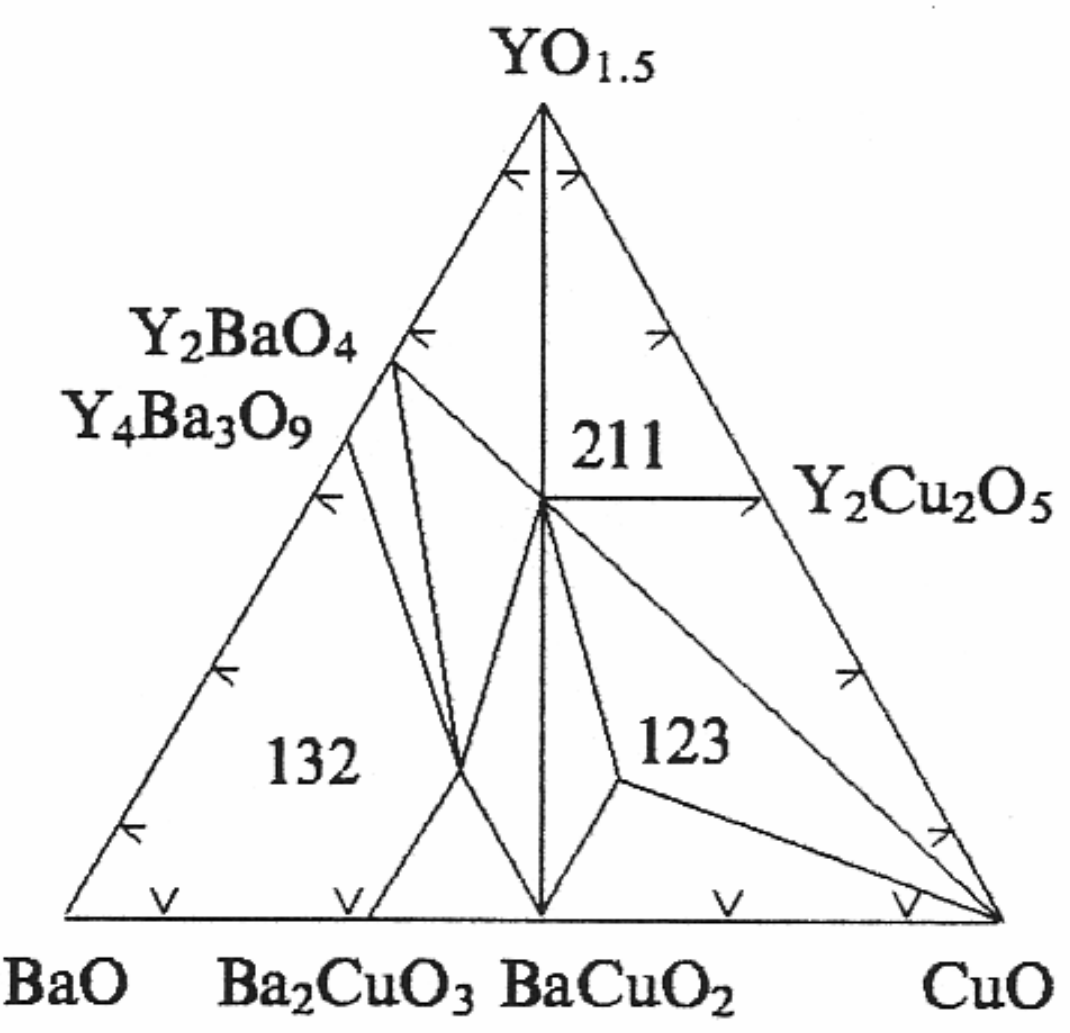

Figure 5 


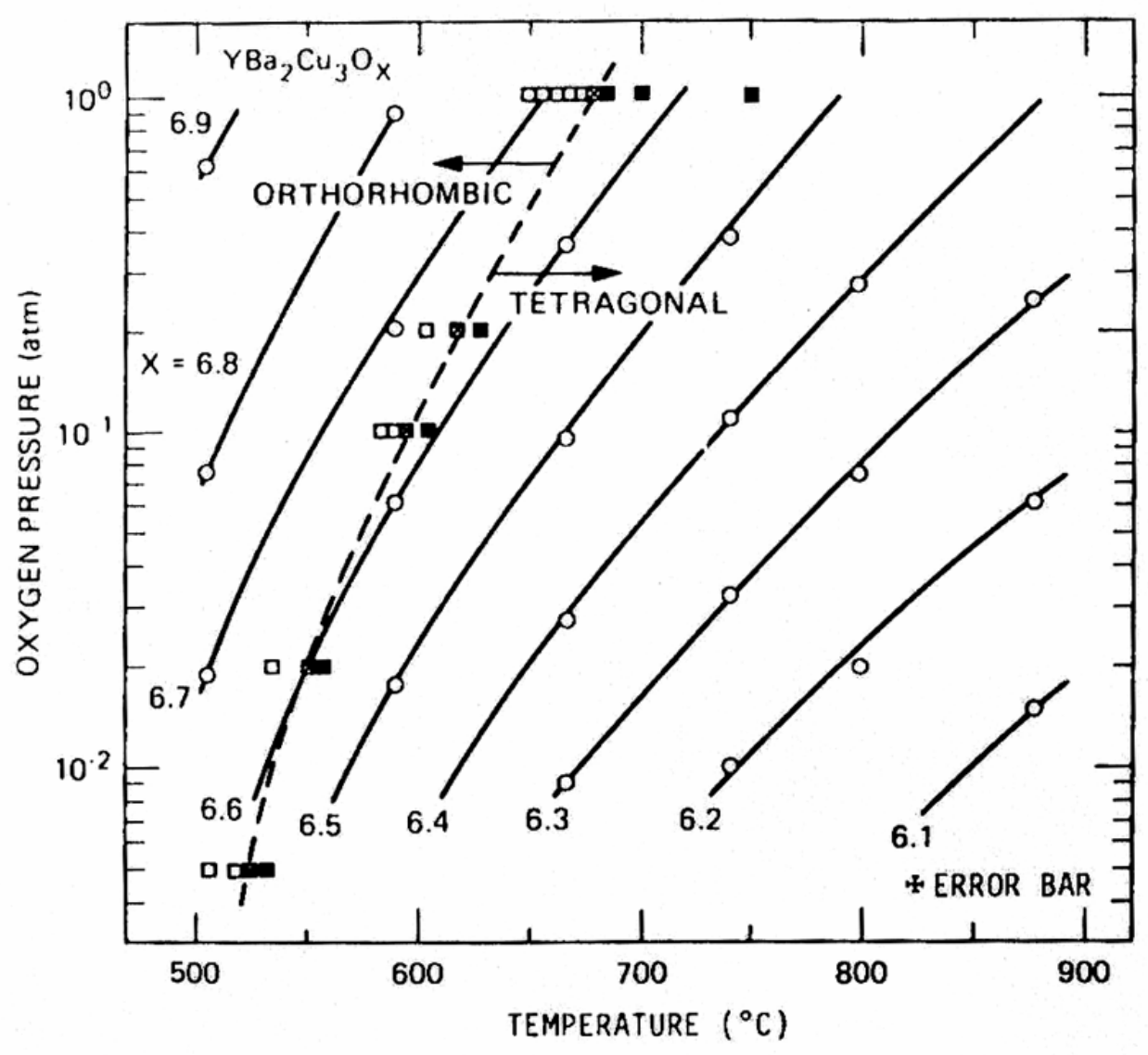

Figure 6 


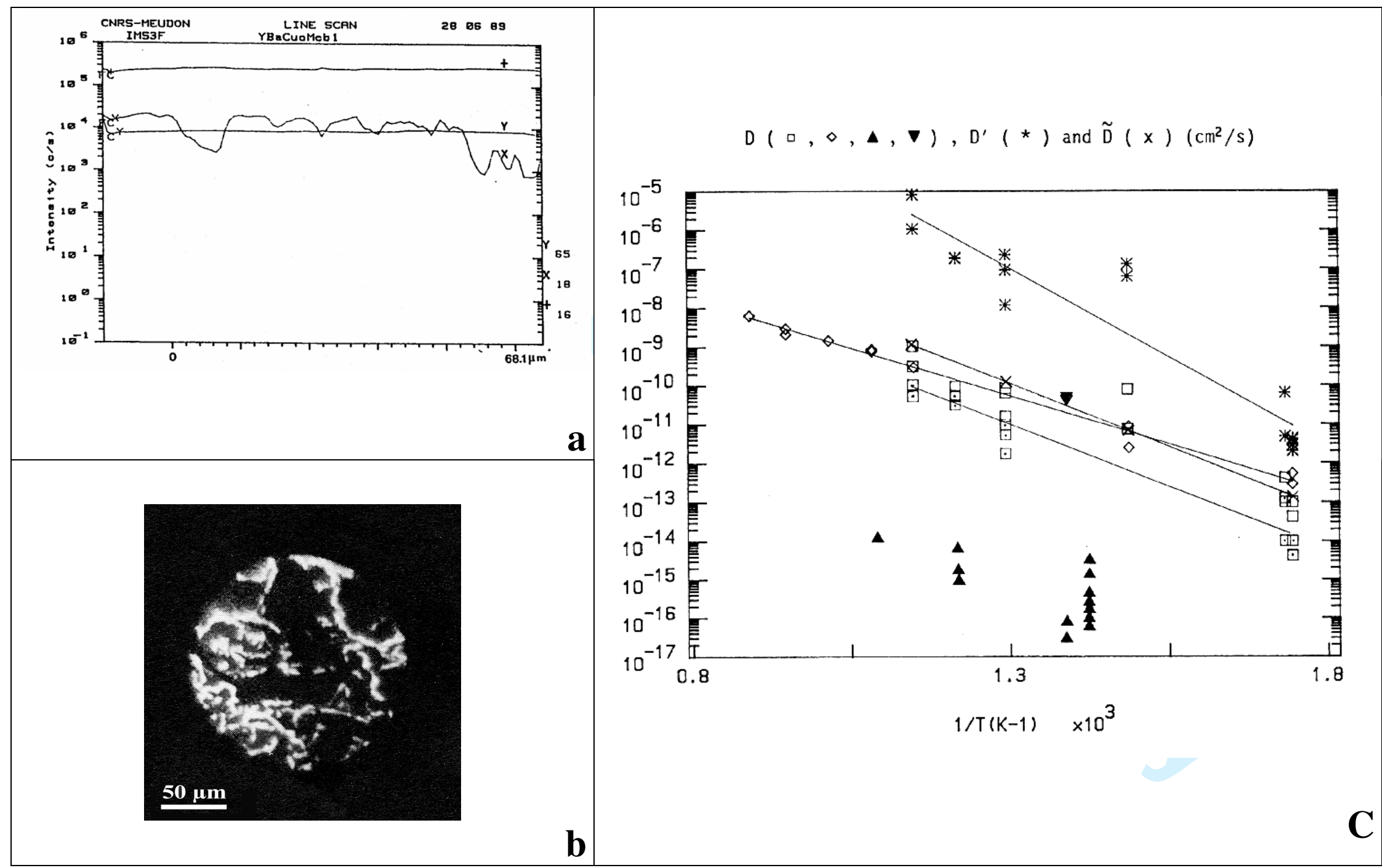

Figure 7 


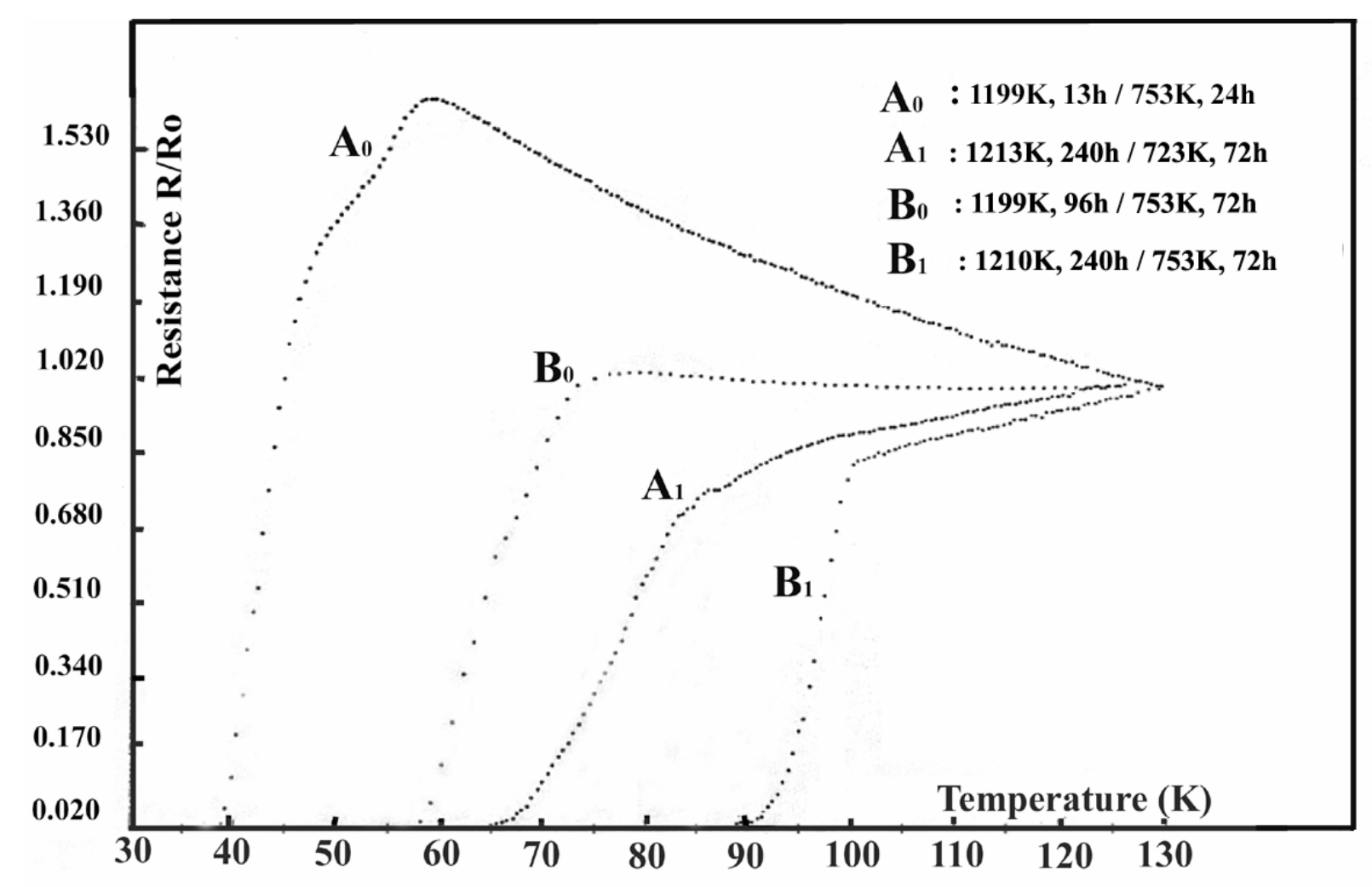

Figure 8 

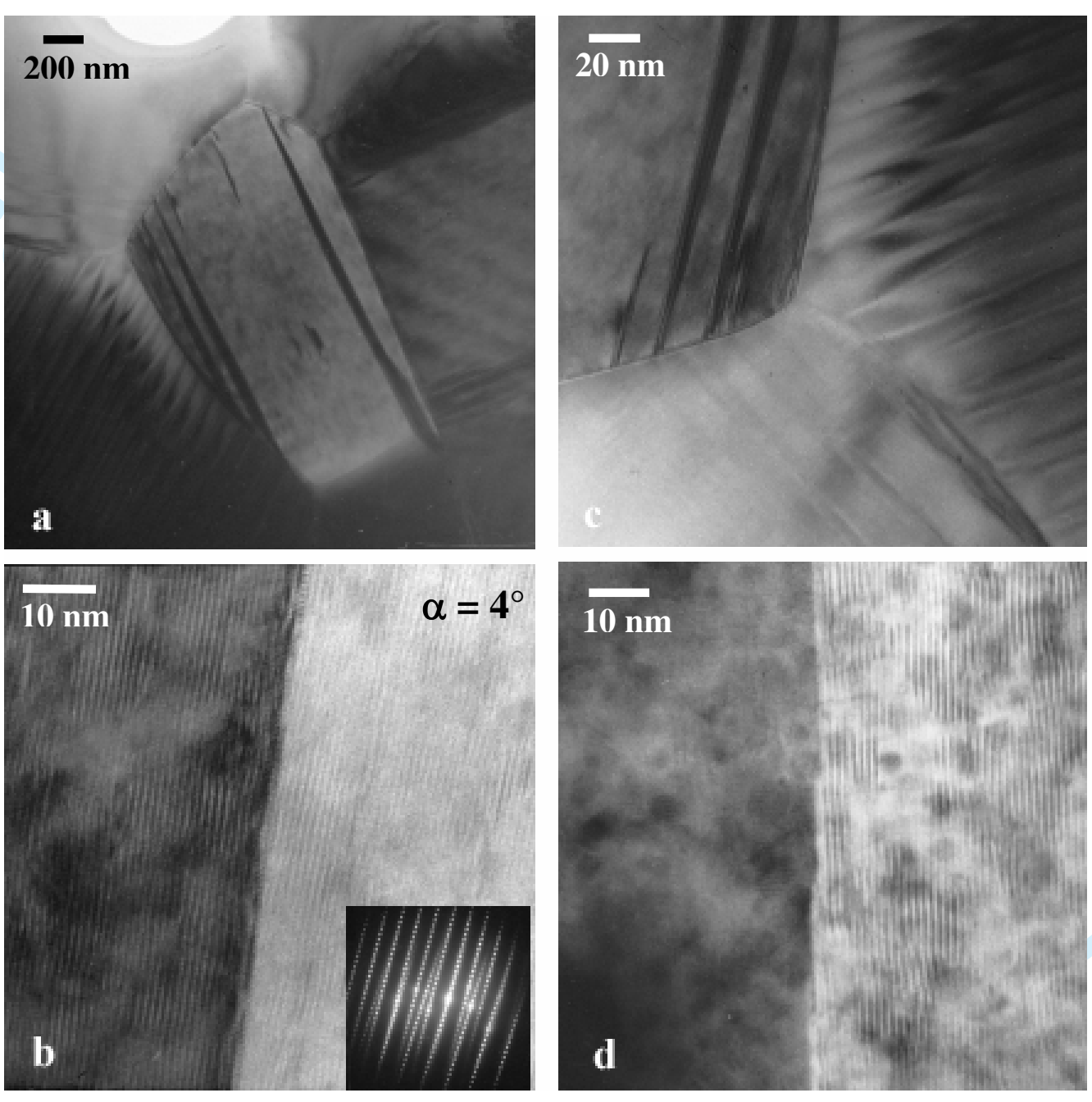

Figure 9 


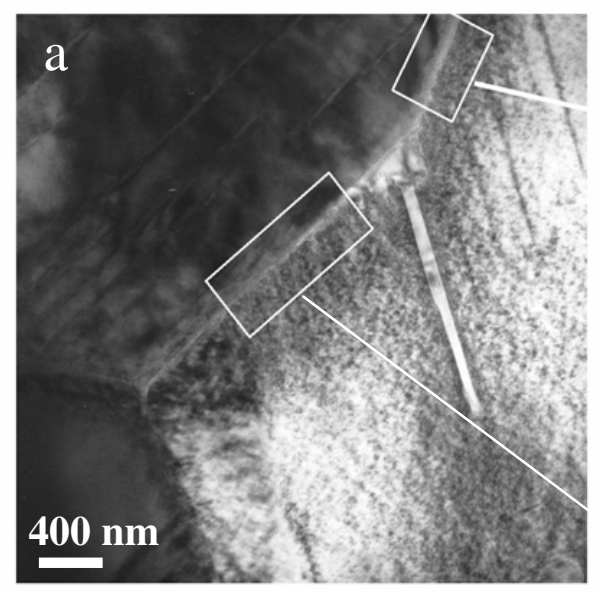

$\mathrm{BaO}$ intergraular grain boundary
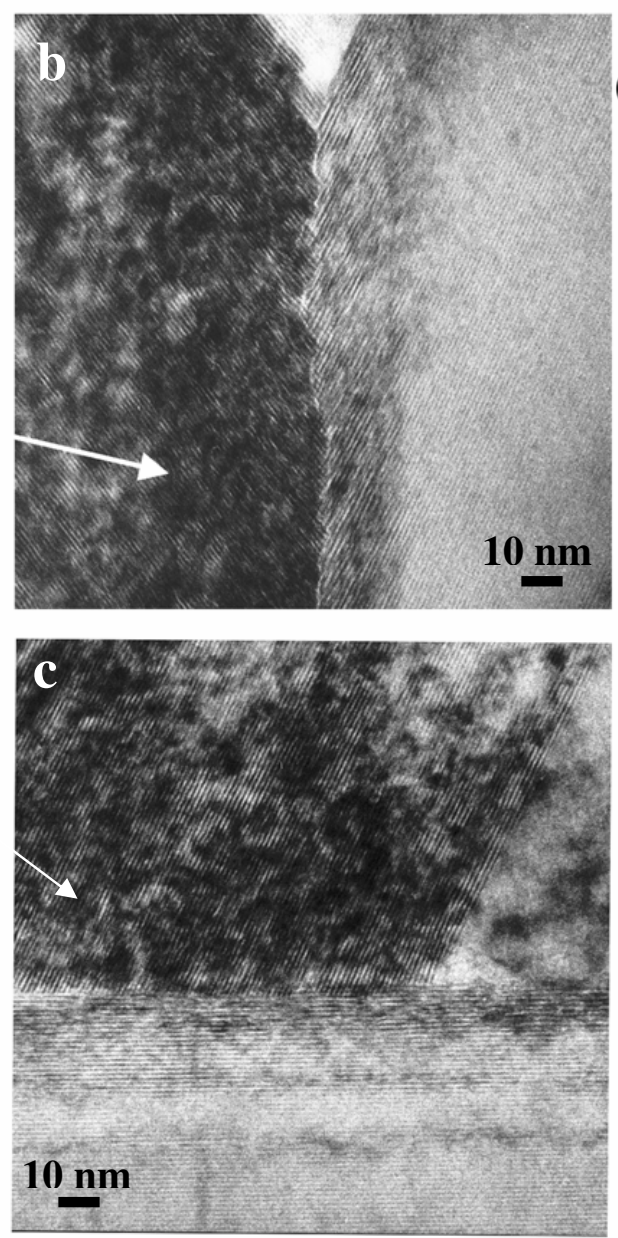

Figure 10

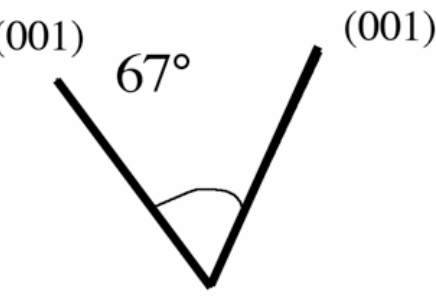

Symmetric

facetted GB exhibiting (001) planes

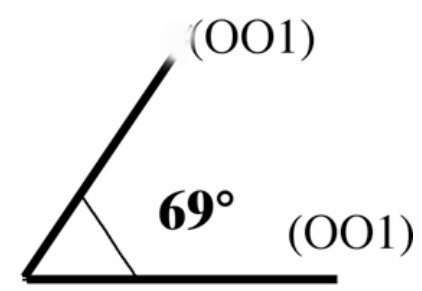

aymmetric GB exhibiting (001) planes 


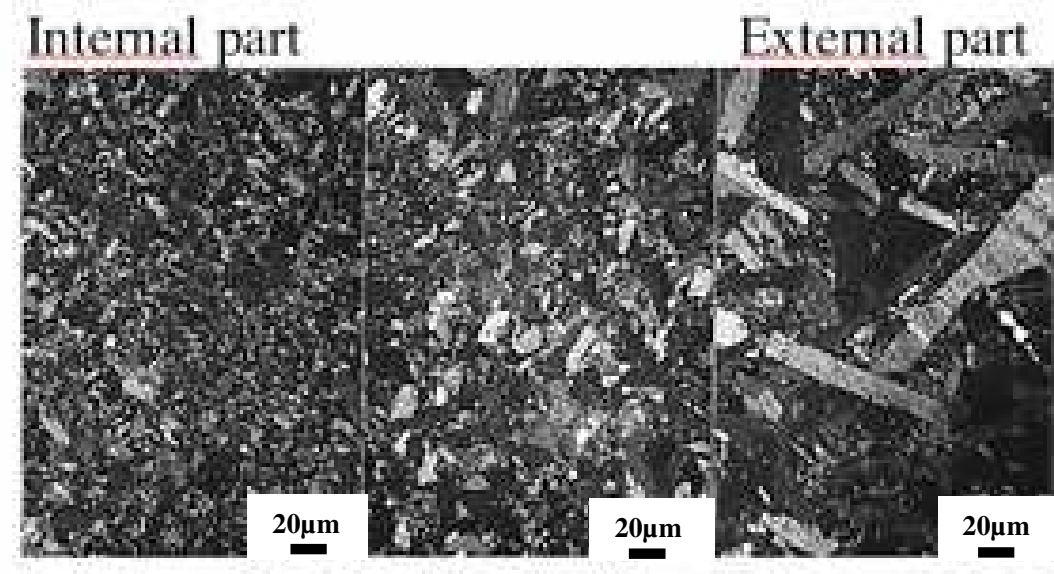

[001] zone axis

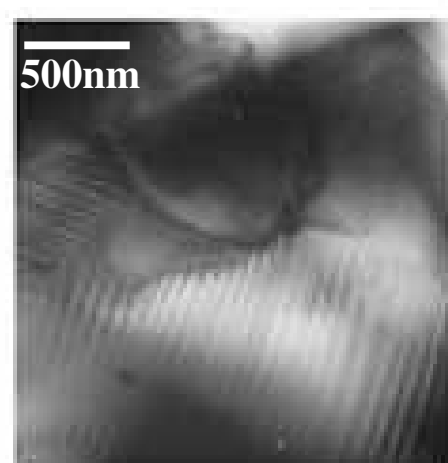

a

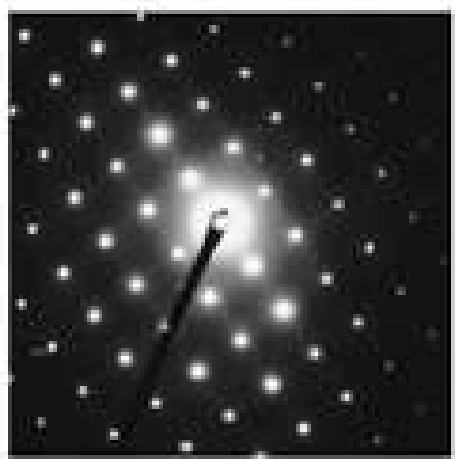

d
Internal part Extemal part

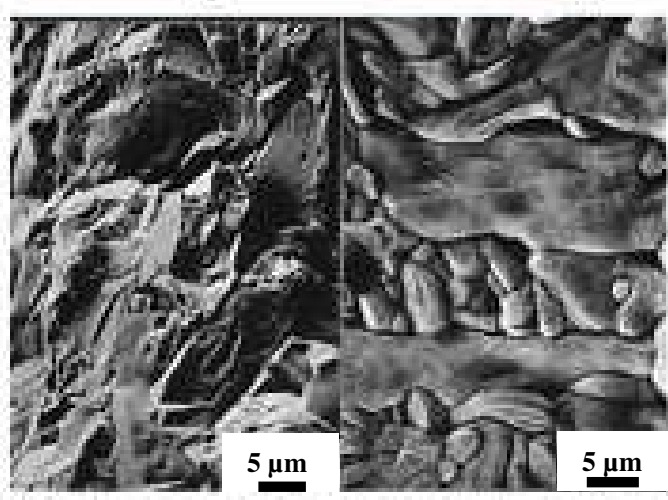

b
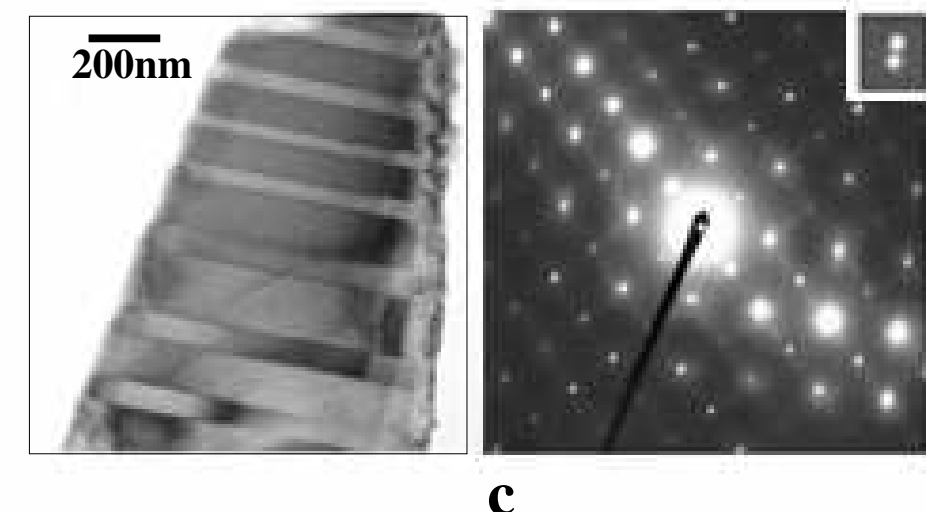

Figure 11 


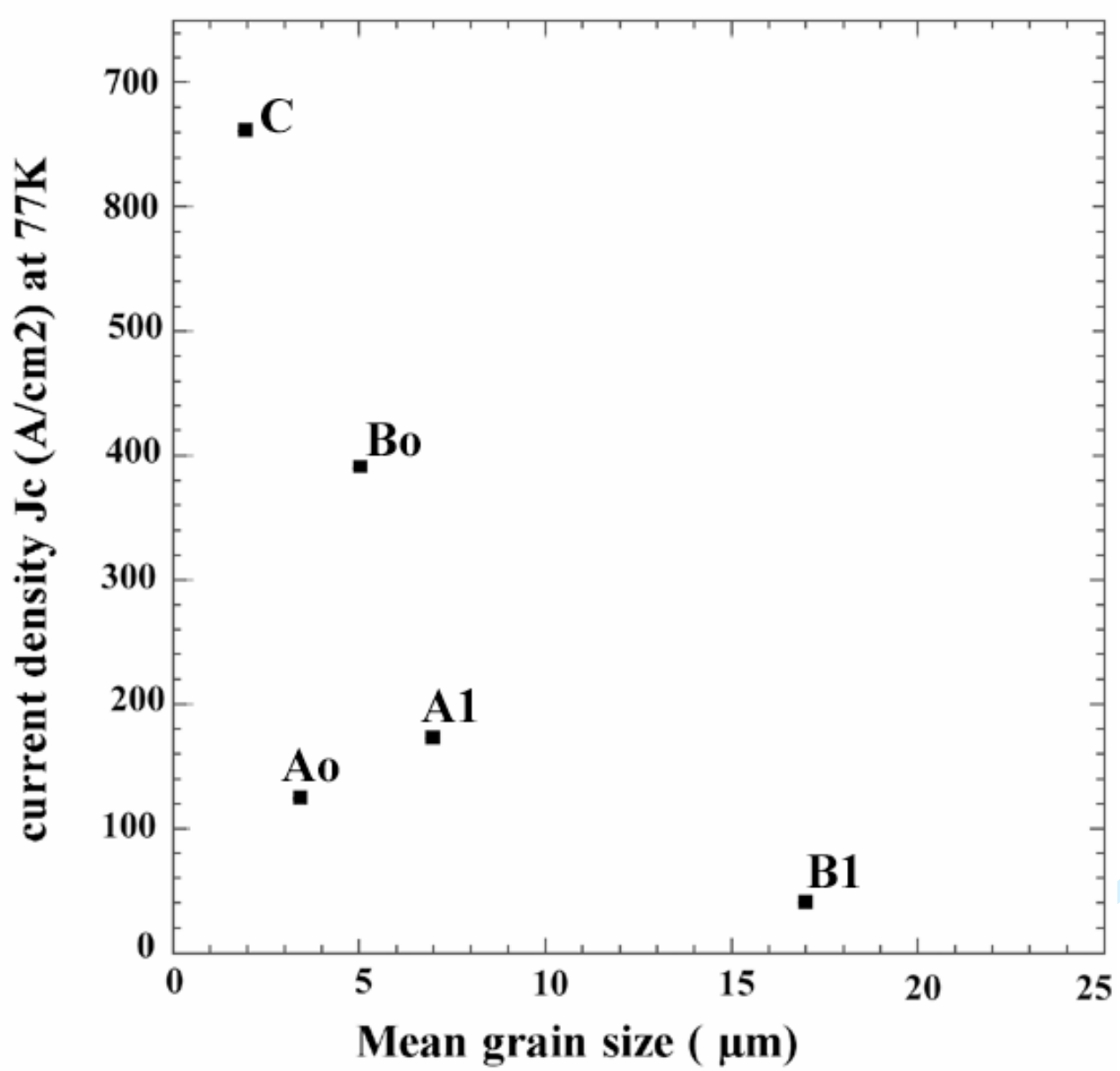

Figure 12 


\section{Figure 13}
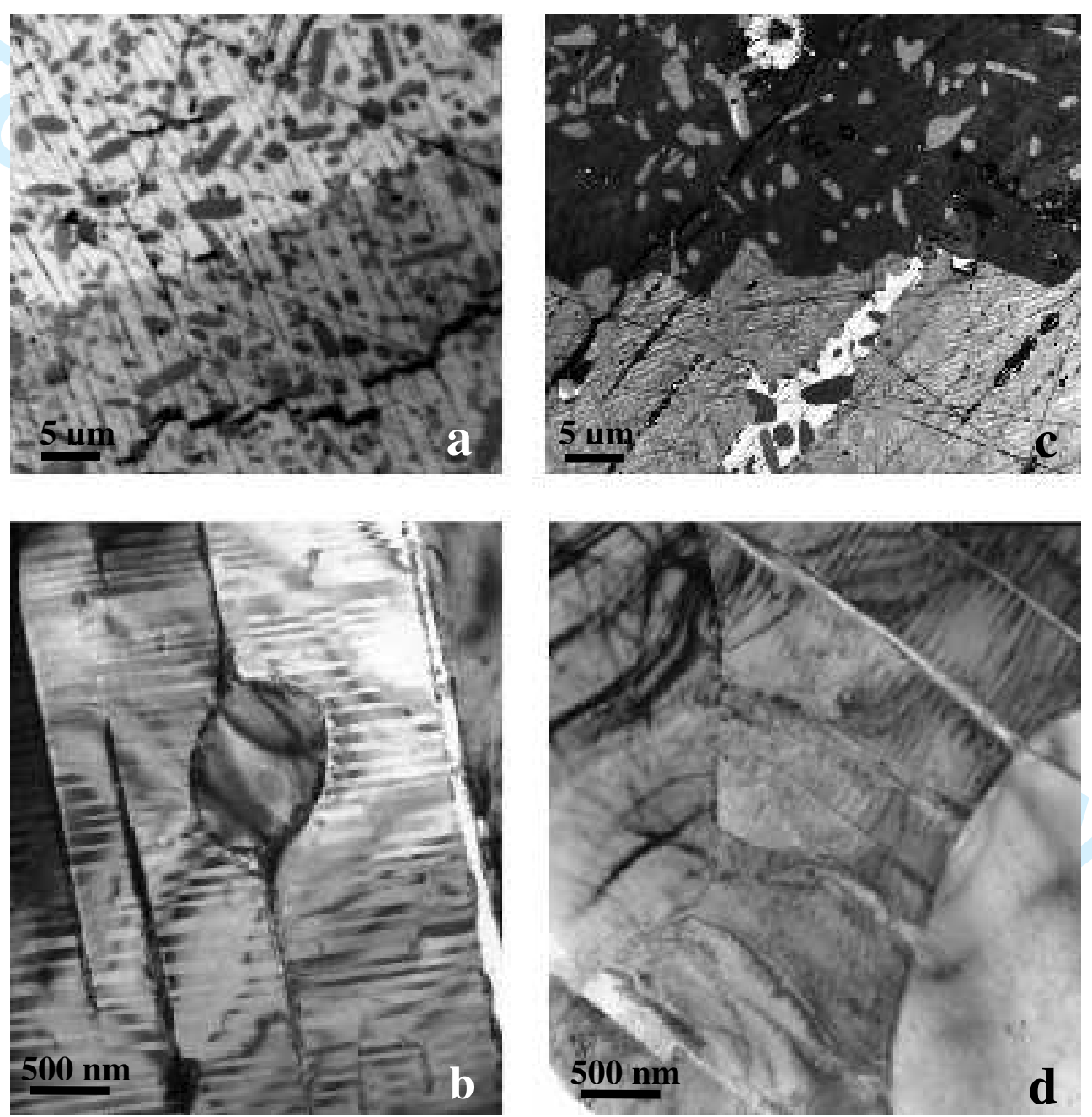

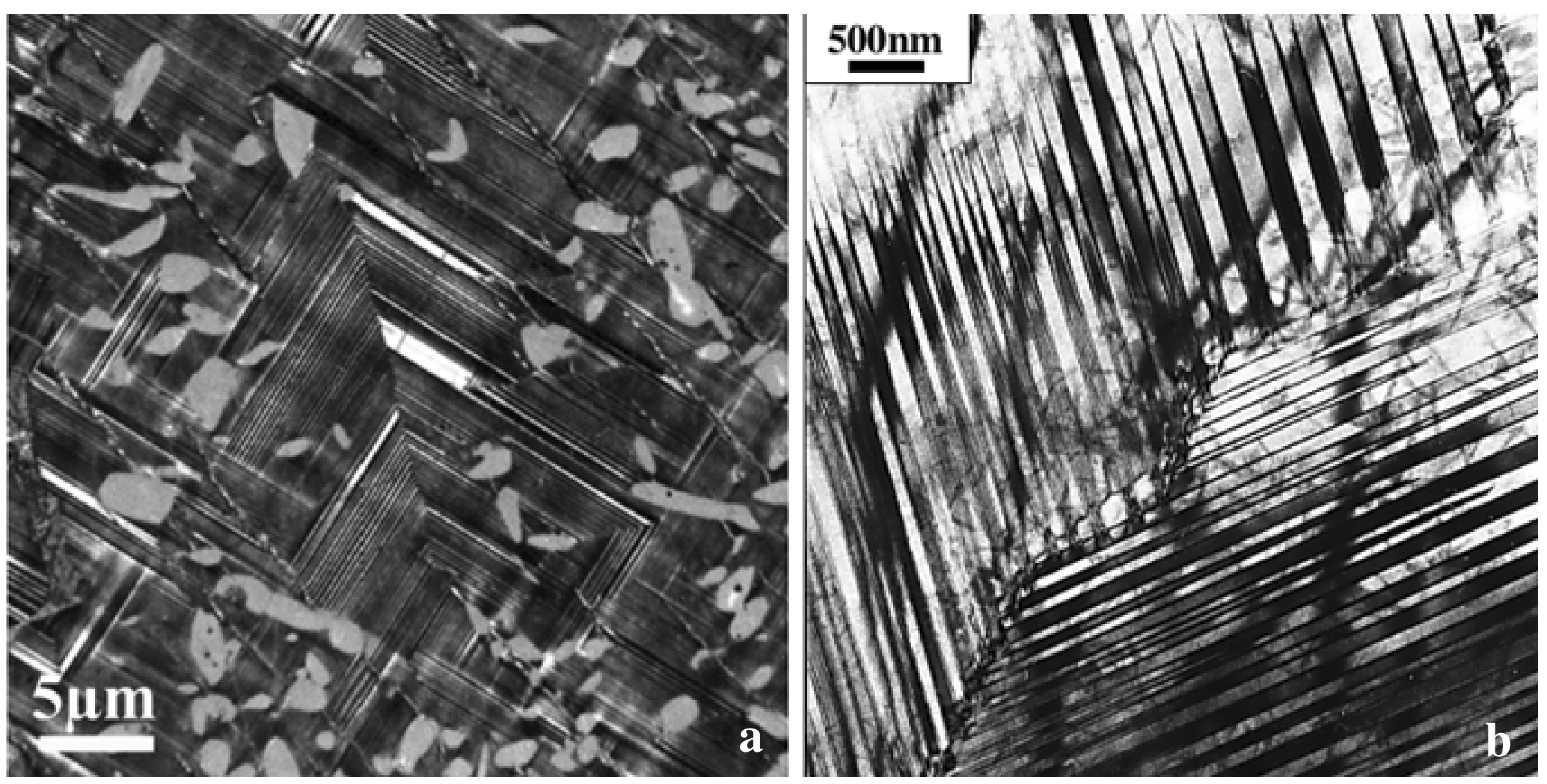

Figure 14

http://mc.manuscriptcentral.com/pm-pml 


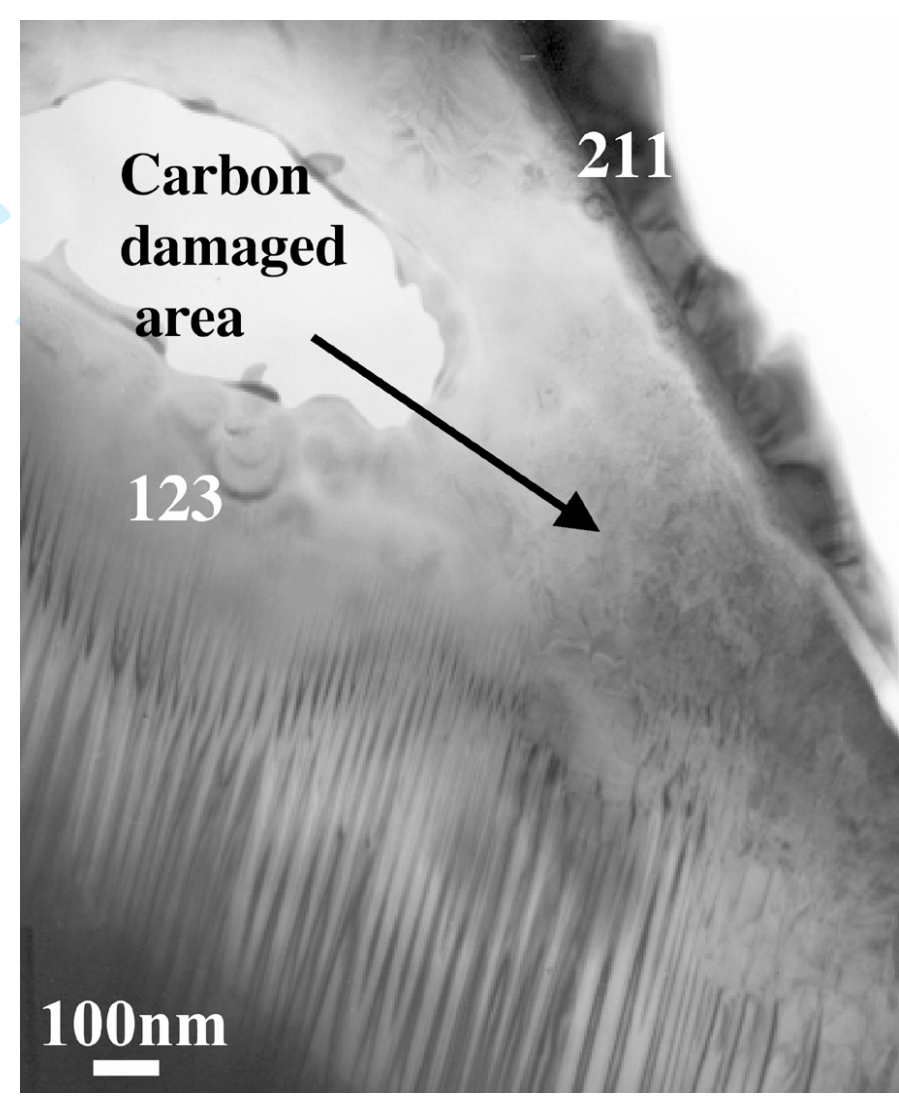

Figure 15 


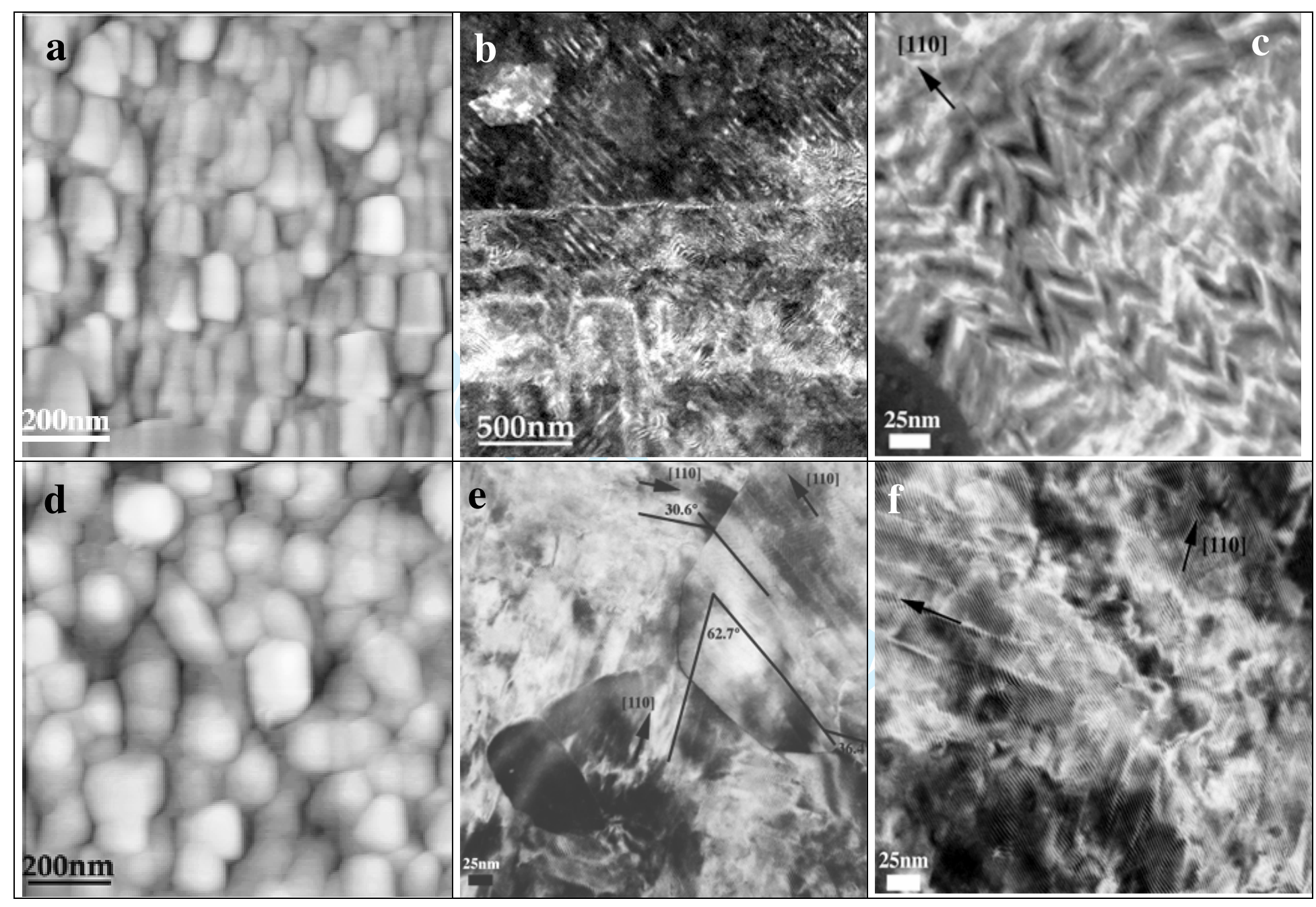

Figure 16 


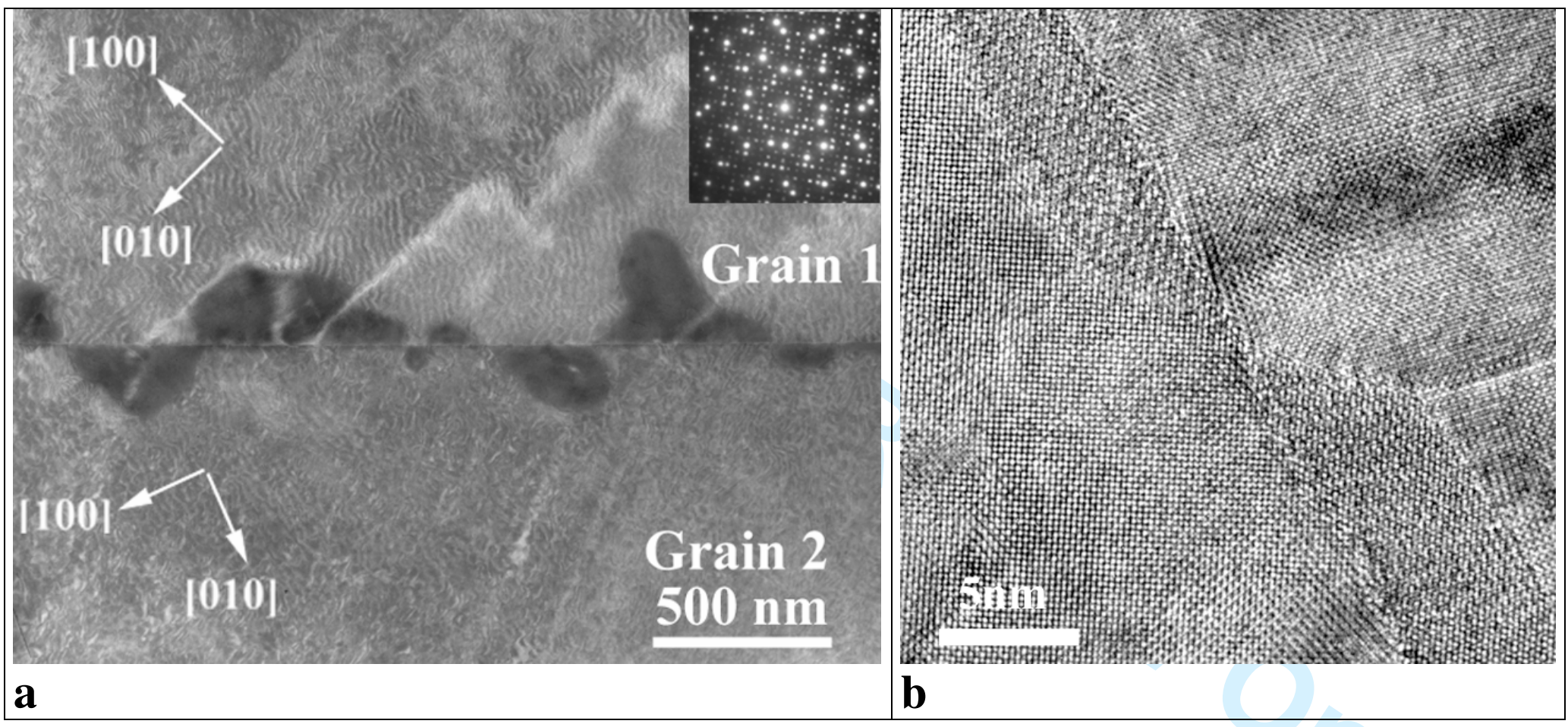

Figure 17 


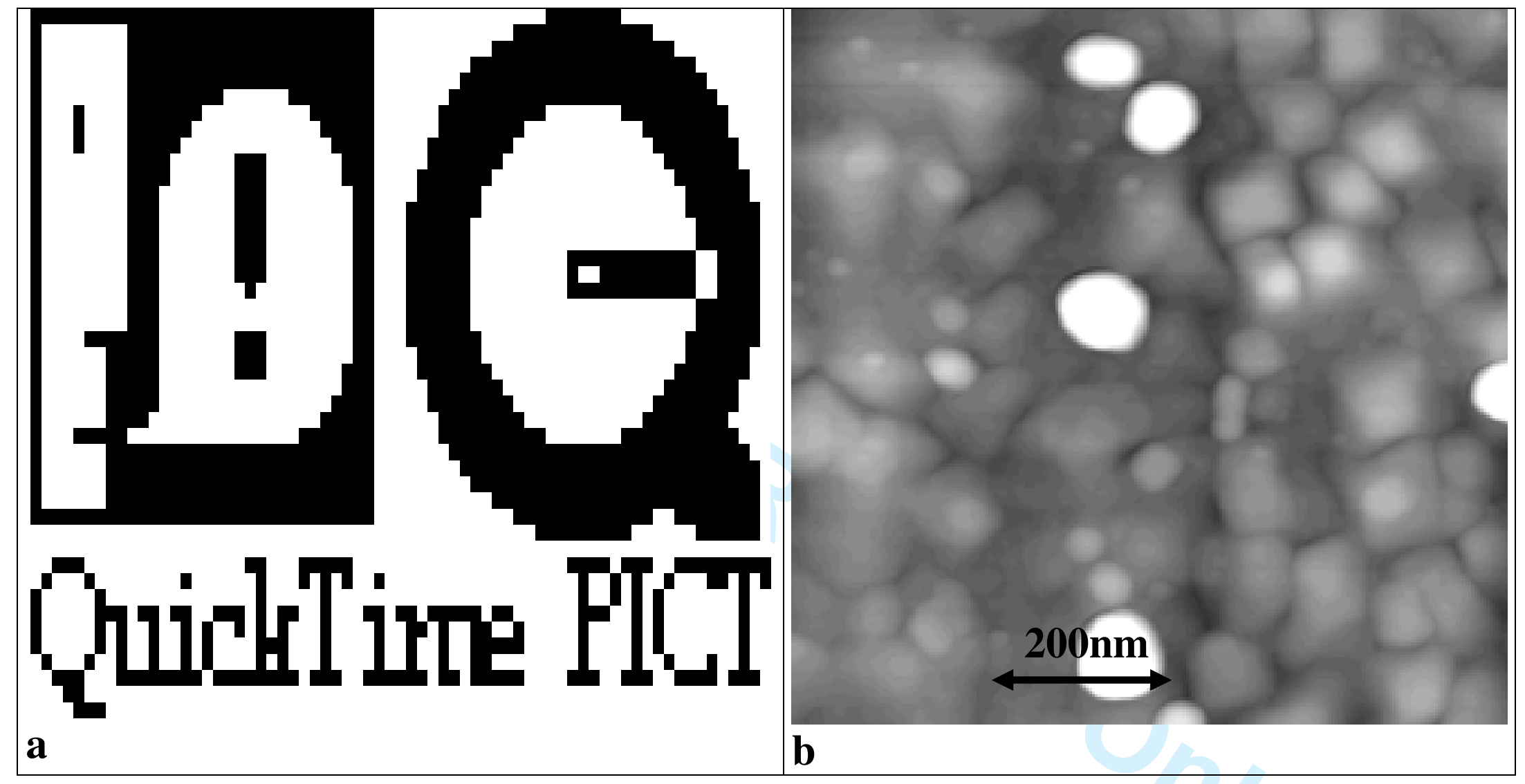

Figure 18 


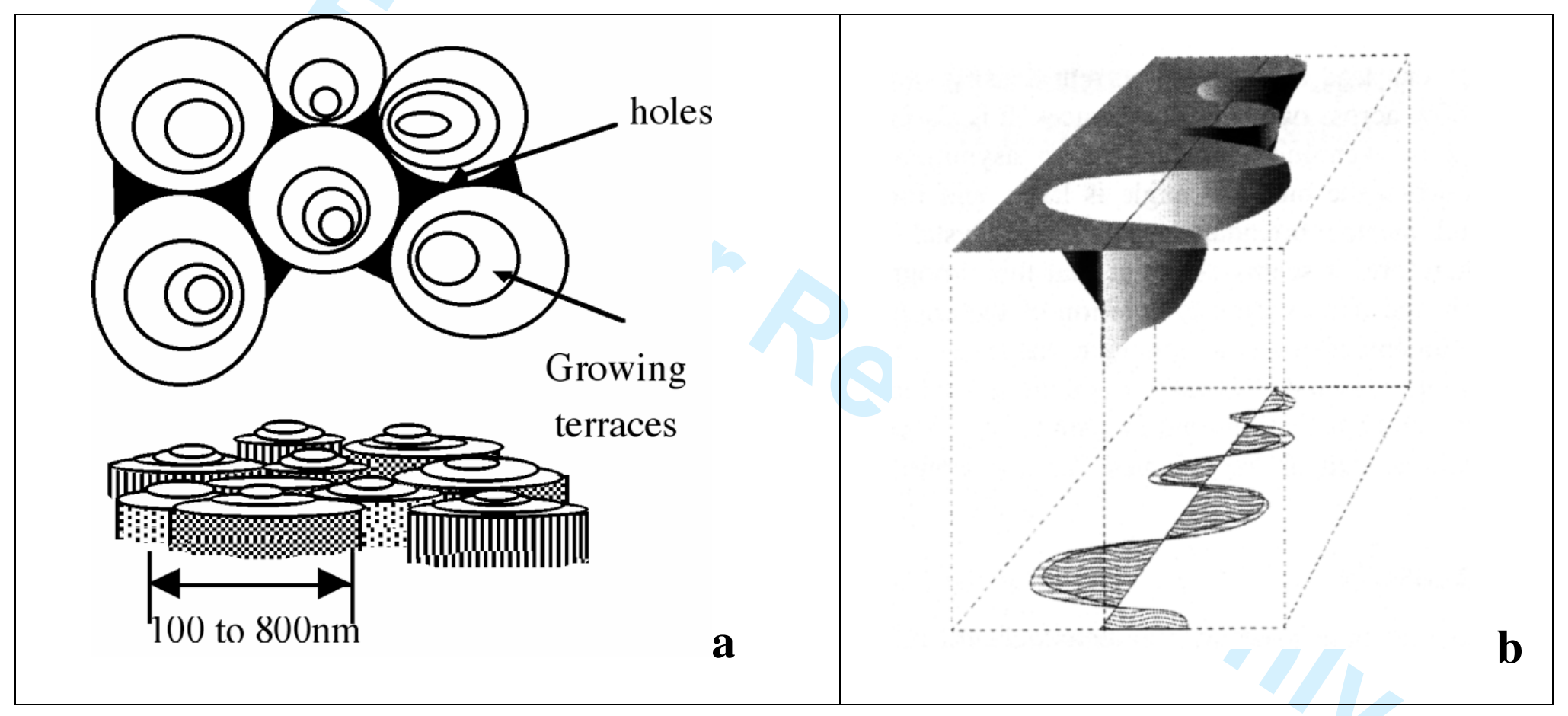

Figure 19 


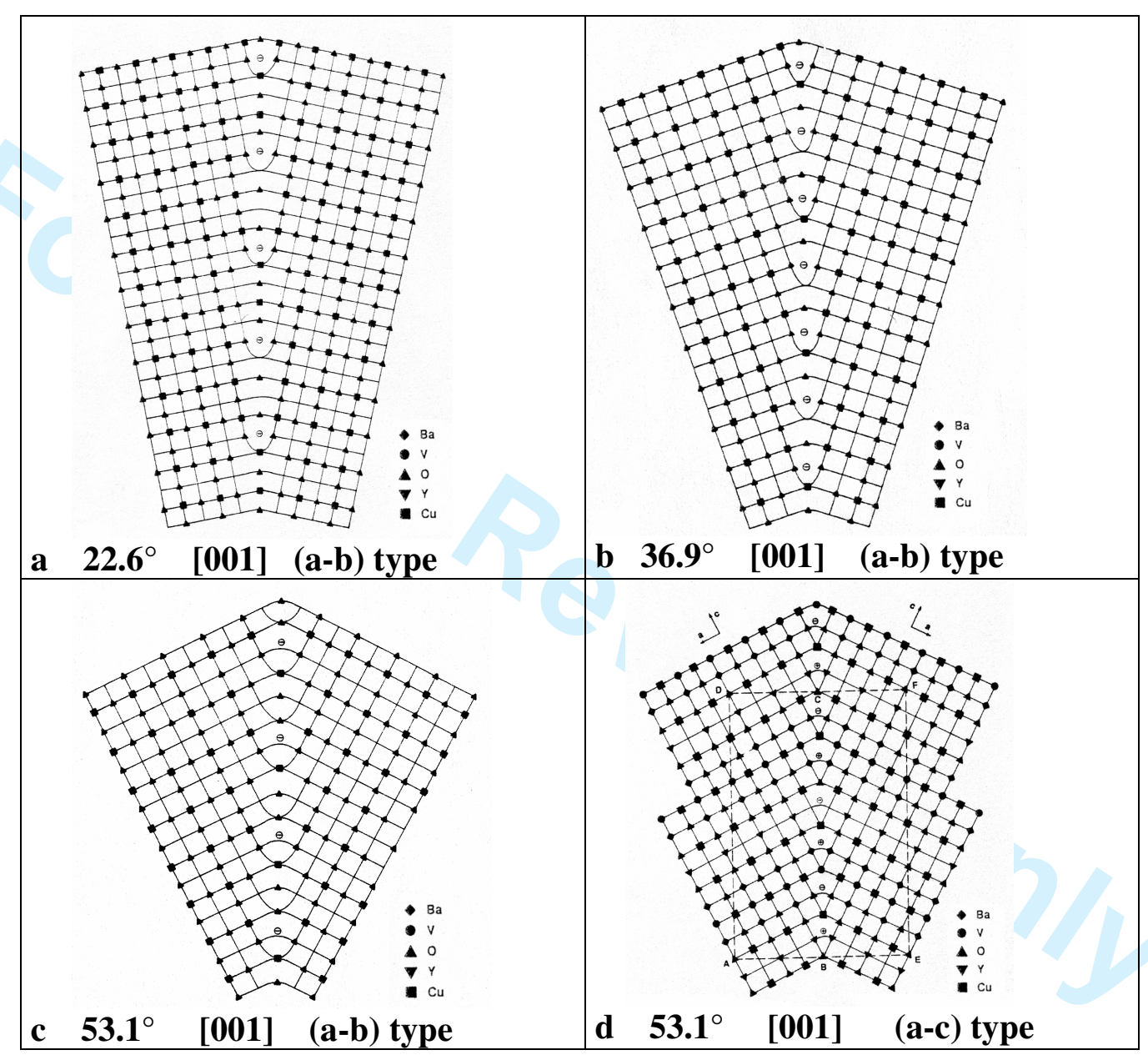

Figure 20 


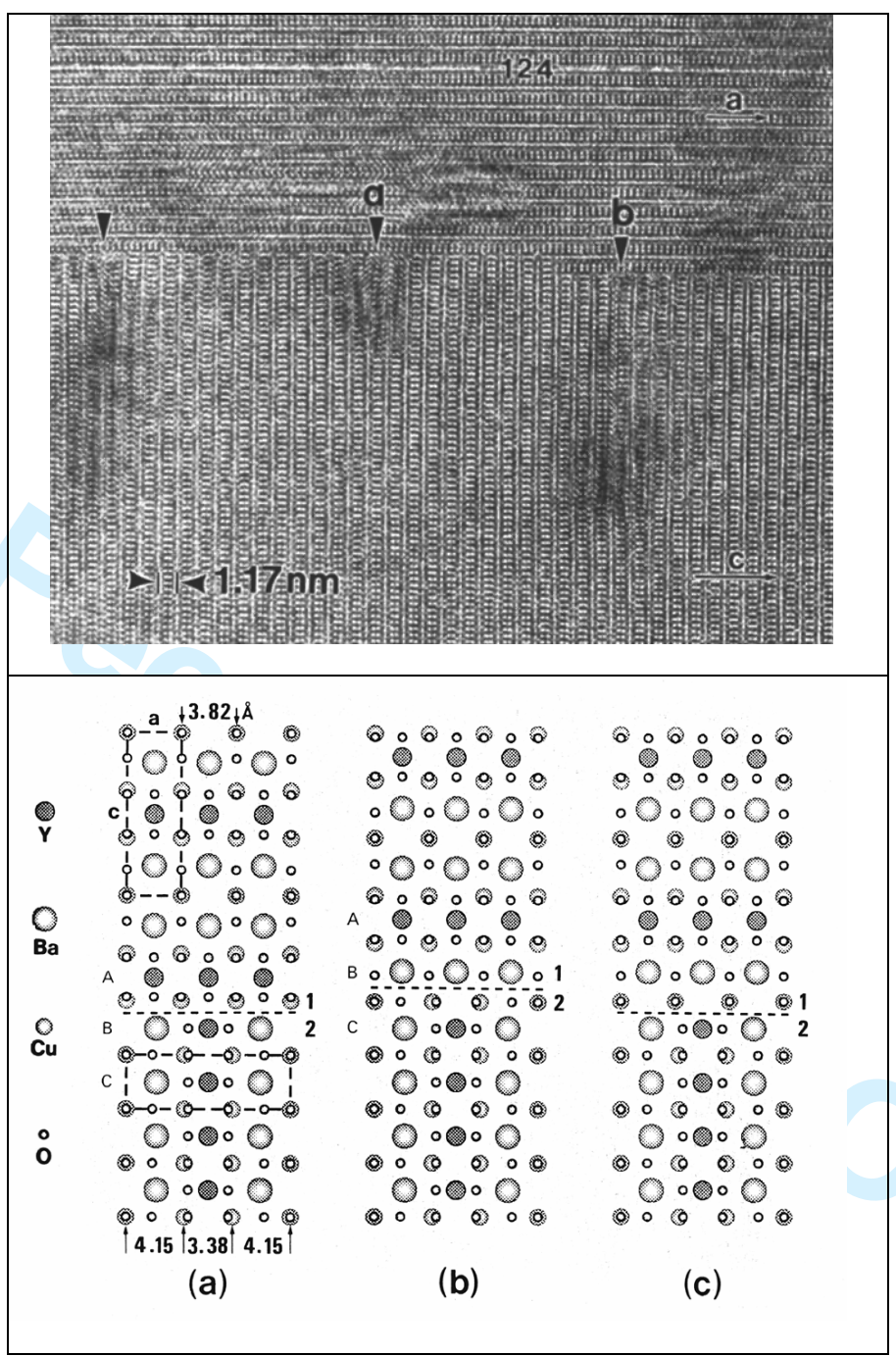

Figure 21 


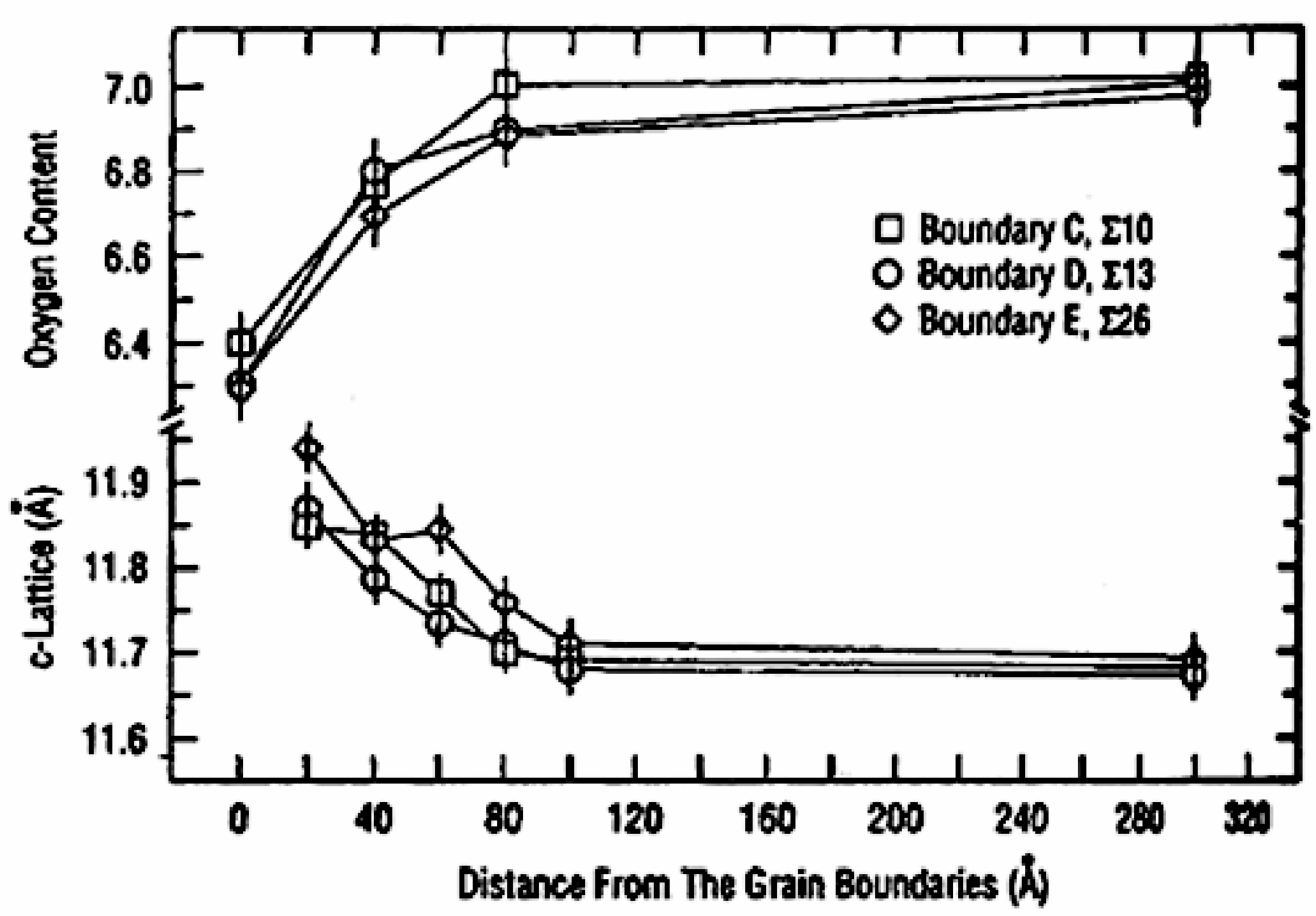

Figure 22 


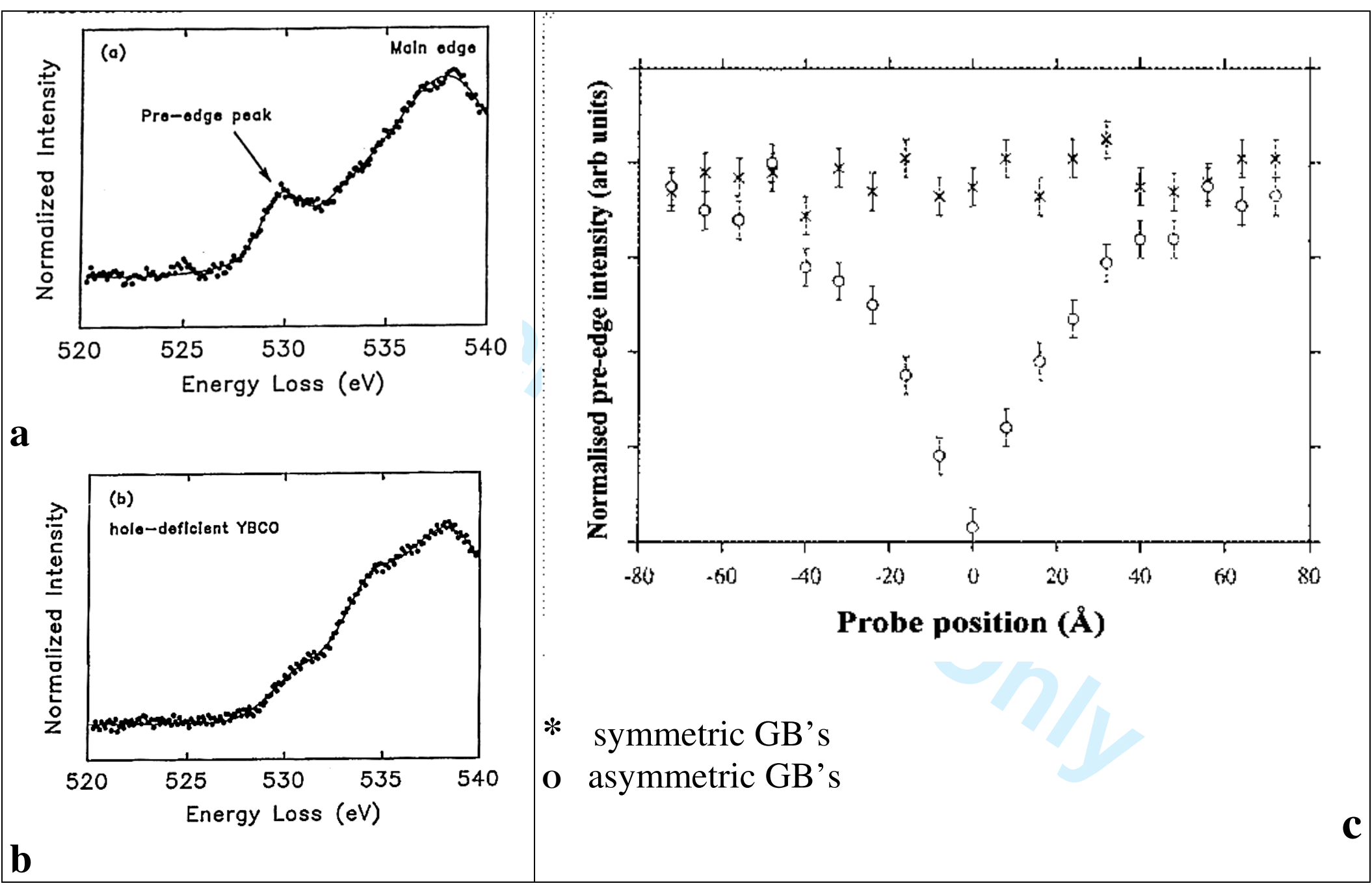

Figure 23 


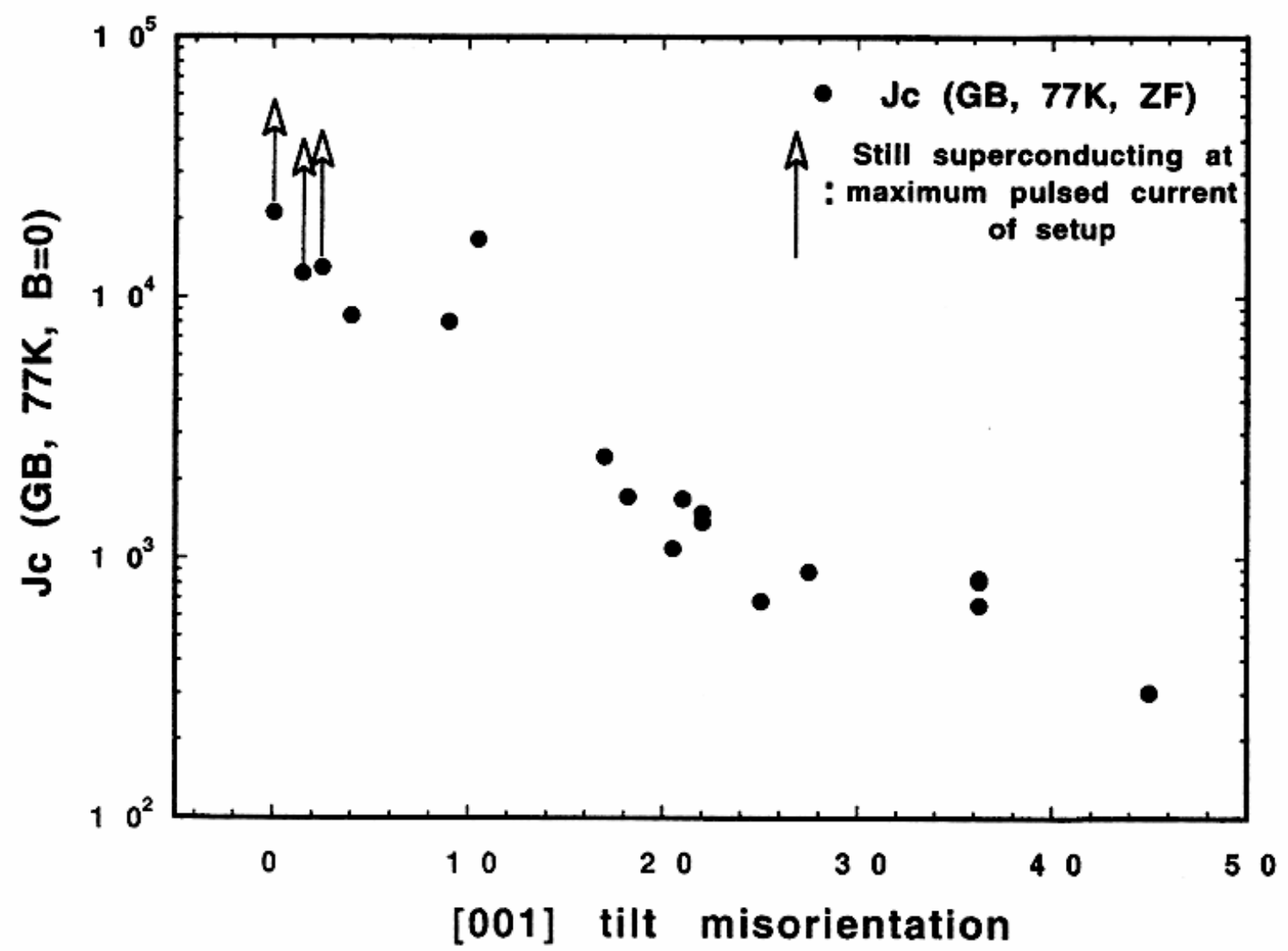

Figure 24 


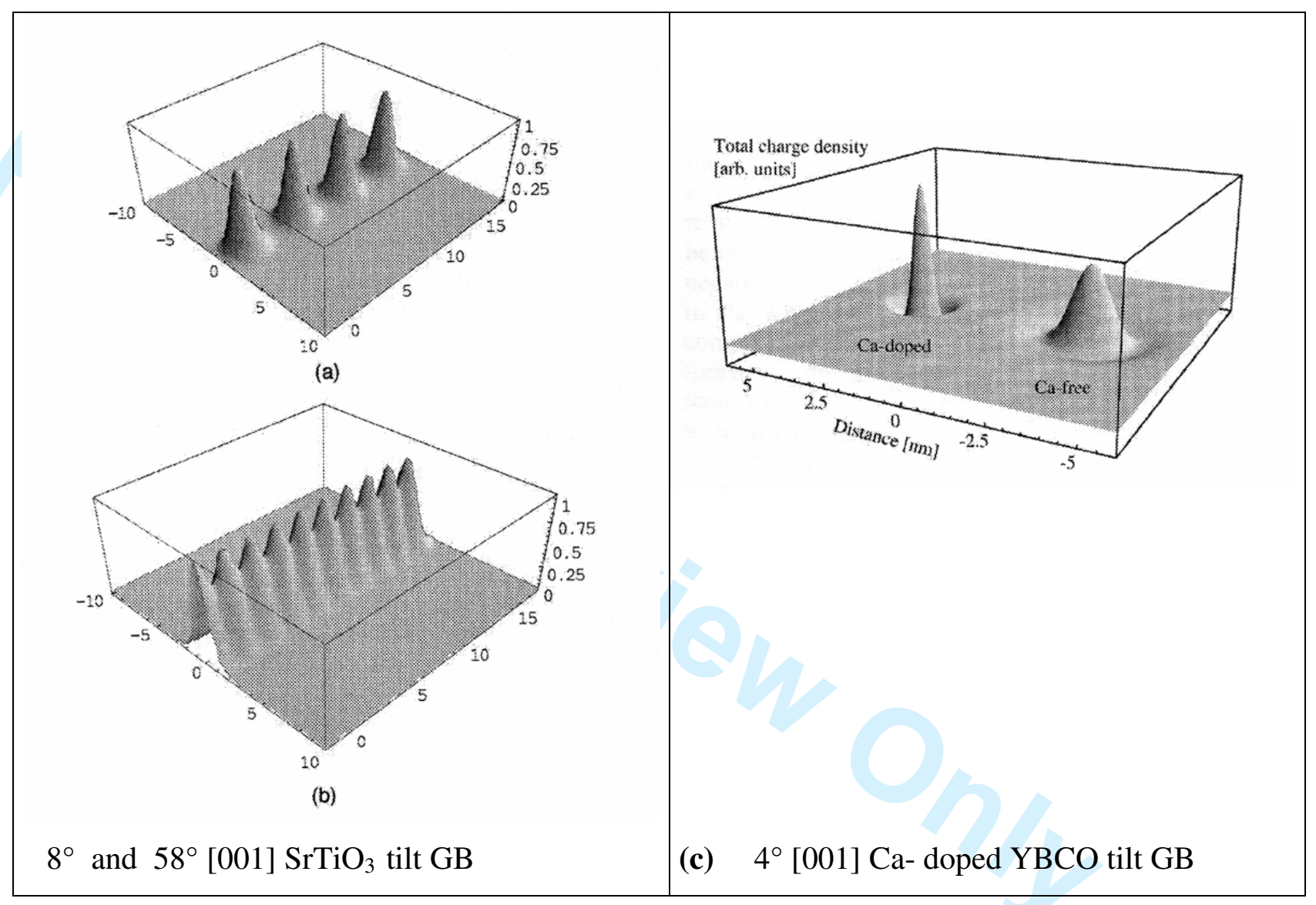

Figure 25 


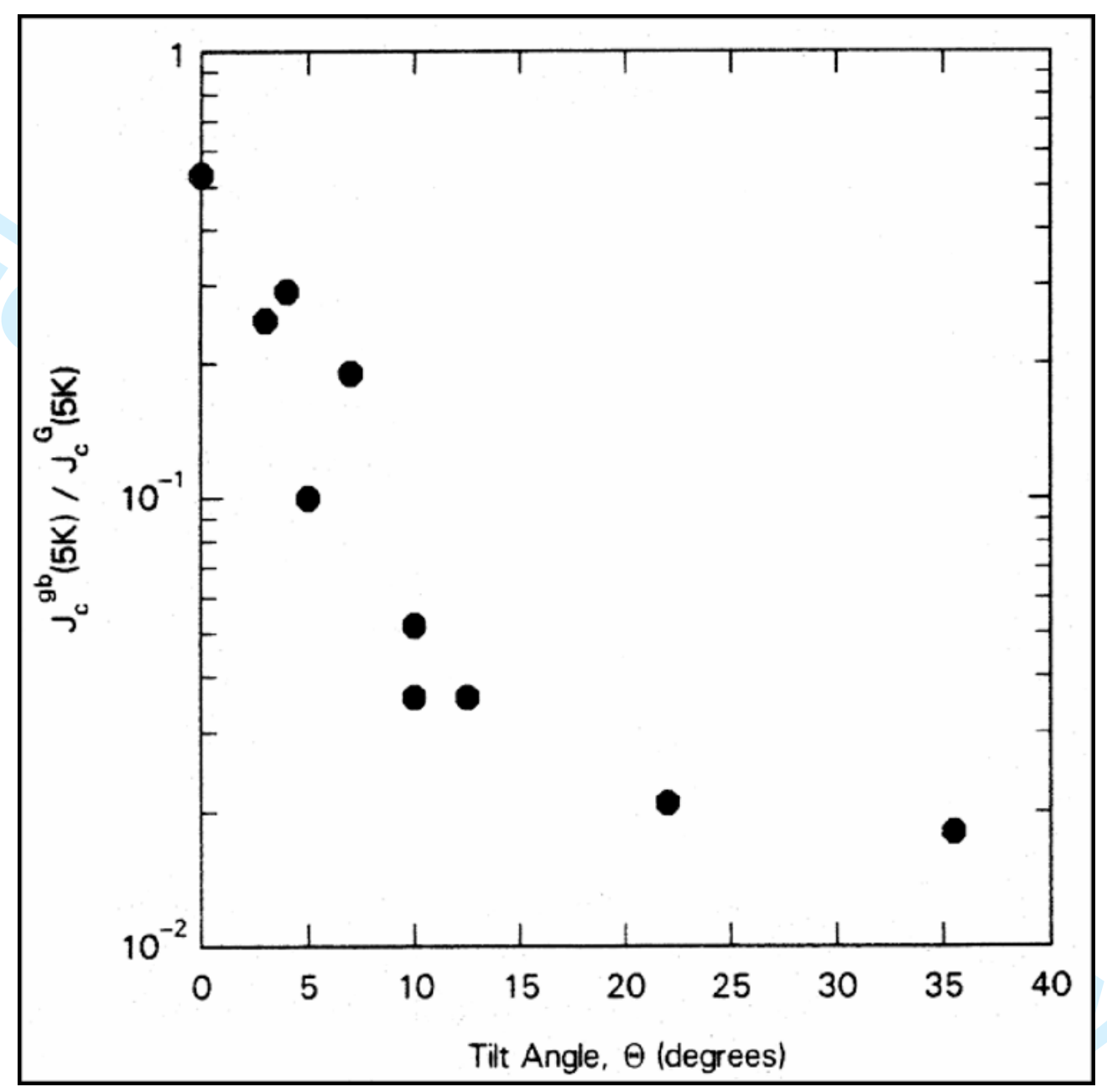

Figure 26 


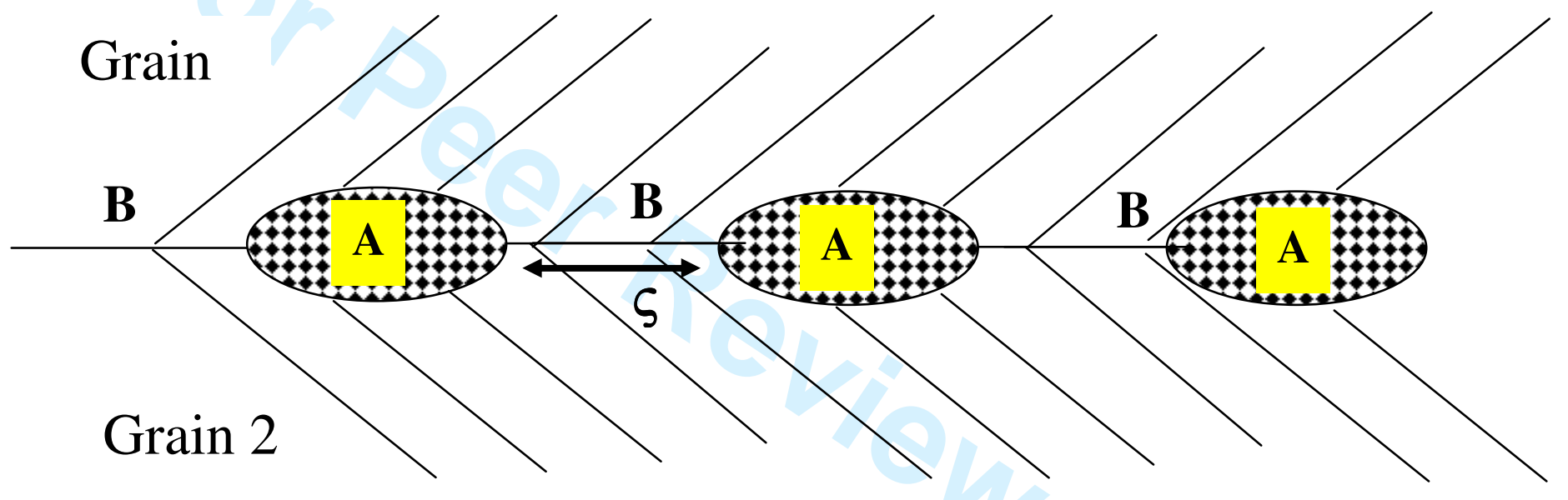

Figure 27 


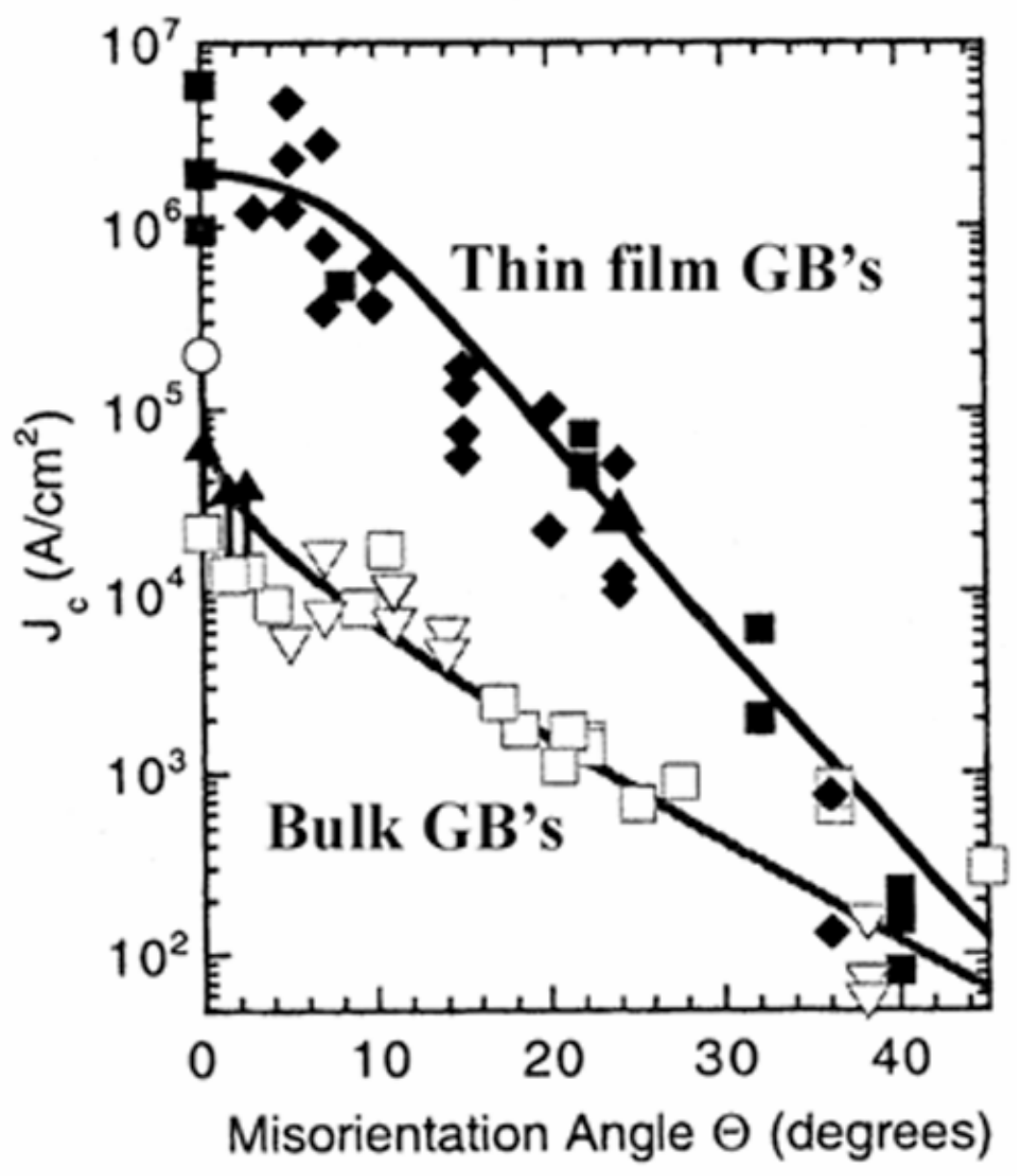

Figure 28 


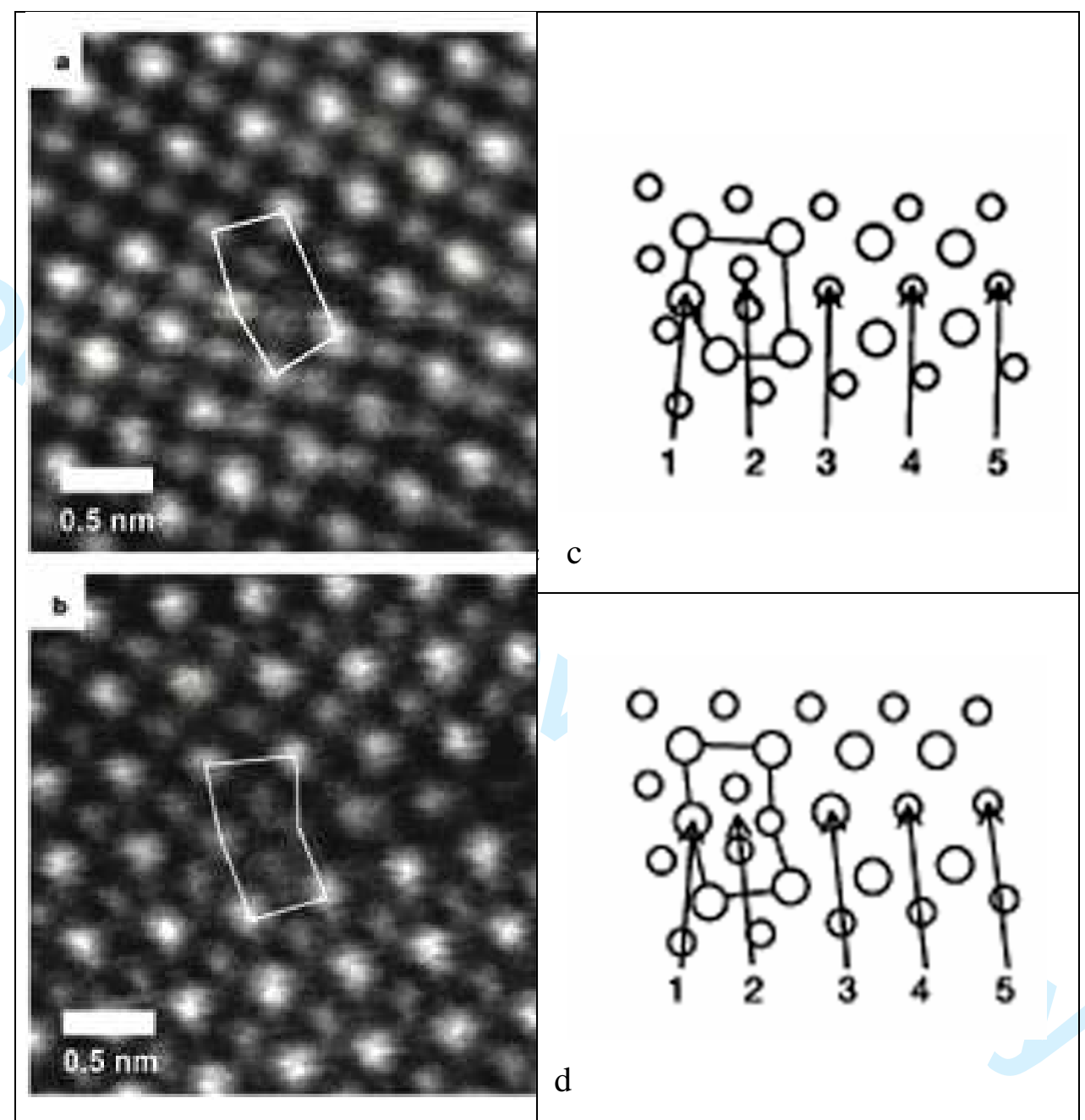

Figure 29 


$$
\text { styot }
$$




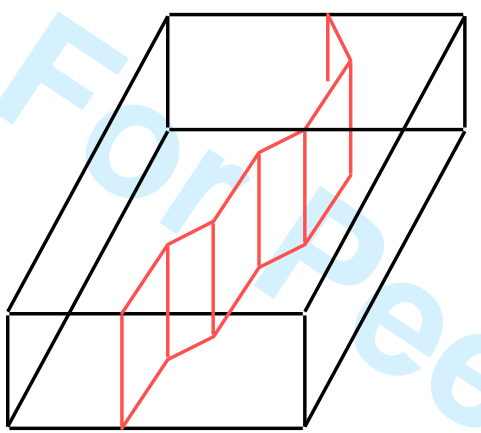

Facetted GB plane

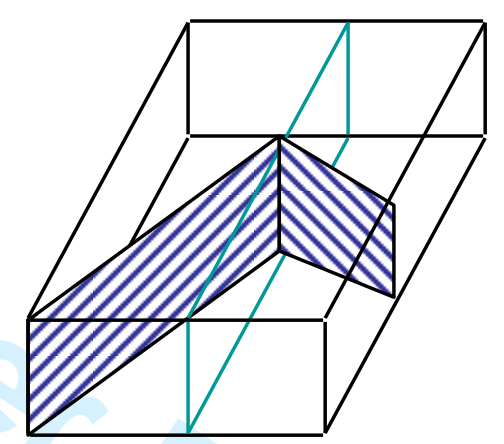

Asymmetric GB

Figure 31a

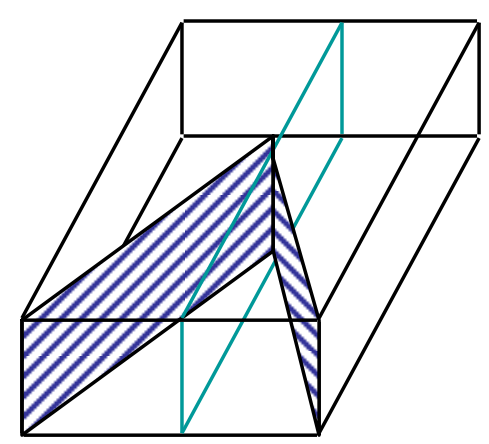

Symmetric GB 


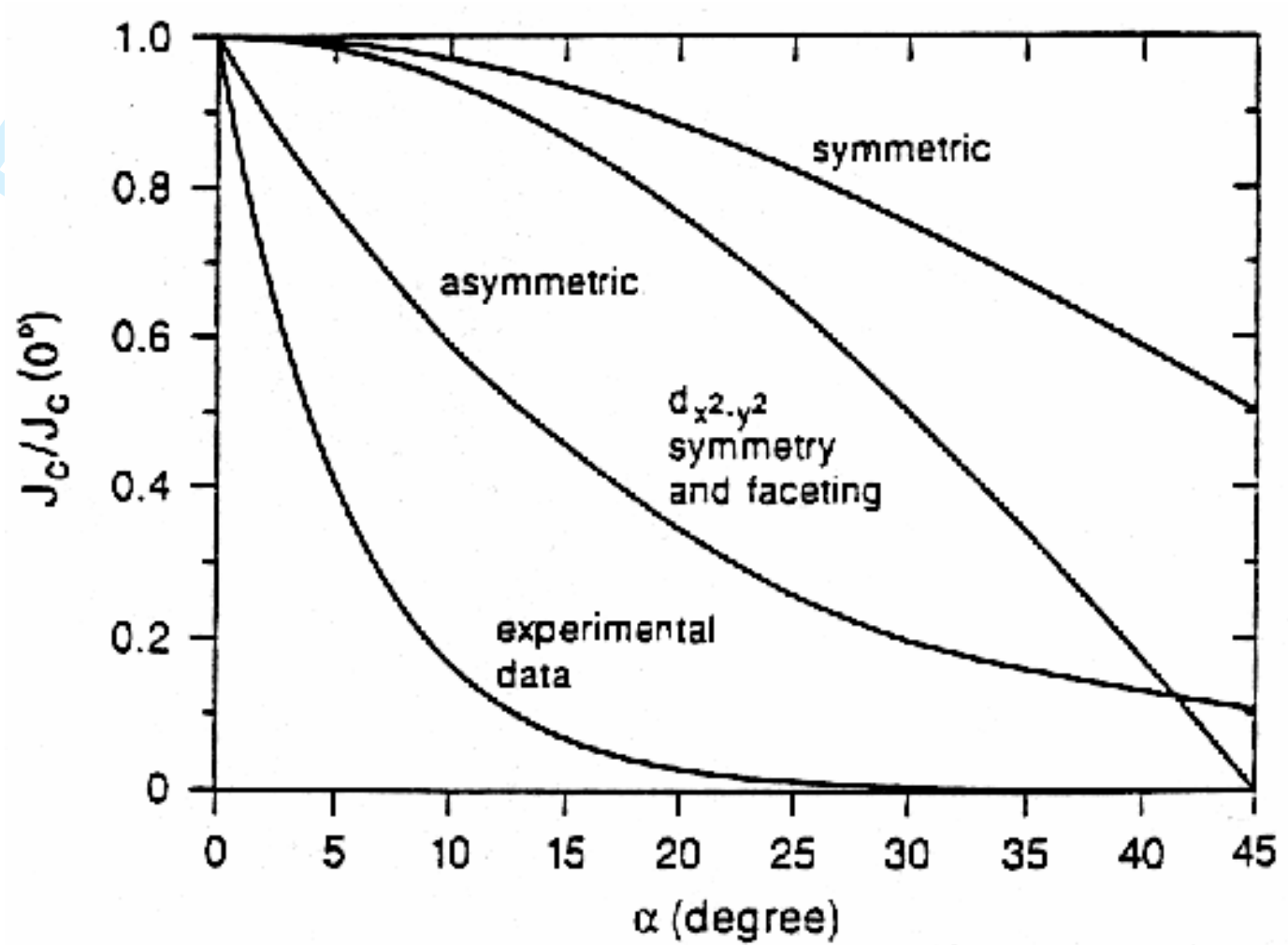

Figure 31b 


\begin{tabular}{|c|c|c|c|}
\hline $\begin{array}{c}\text { YBCO } \\
\text { Structures }\end{array}$ & $\begin{array}{l}\text { Oxygen } \\
\text { contents }\end{array}$ & Tc $(K)$ & $\begin{array}{c}\text { Lattice } \\
\text { Parameters }(\mathbf{n m})\end{array}$ \\
\hline Ortho I & $\begin{array}{c}\mathbf{O}_{7}-\mathbf{O}_{6.9} \\
\mathbf{O}_{6.97}\end{array}$ & $\begin{array}{c}91 \\
\text { maximum } \mathrm{Tc}=92\end{array}$ & $\begin{array}{l}a=0.382 \\
b=0.388\end{array}$ \\
\hline Ortho II & $\mathbf{O}_{6.6}-\mathbf{O}_{6.7}$ & 60 & $\begin{array}{c}c=1.168 \\
+ \text { oxygen ordering* }\end{array}$ \\
\hline Tetragonal & $\mathrm{O}_{6.5}-\mathrm{O}_{6.4}$ & $\begin{array}{l}0 \\
0\end{array}$ & $\begin{array}{c}a=b=0.386 \\
c=1.171 \\
+ \text { oxygen ordering* }\end{array}$ \\
\hline
\end{tabular}

\section{Table 1}




\begin{tabular}{|c|c|c|c|c|c|}
\hline SAMPLES & SUBSTRATES & $\begin{array}{c}211 \\
\text { GRAIN SIZES }\end{array}$ & Tc $(K)$ & $\begin{array}{c}\Delta \mathrm{Tc} \\
(\mathbf{K})\end{array}$ & $\begin{array}{r}\mathrm{Jc} \mathrm{A} / \mathrm{cm}^{2} \\
\text { at } 77 \mathrm{~K}\end{array}$ \\
\hline $\bar{D}$ & $\mathrm{MgO}$ & $<1 \mu \mathrm{m}$ to $5 \mu \mathrm{m}$ & 82 & 13 & $(\mathbf{H} / / \mathbf{c}) \sim 0$ \\
\hline $\mathbf{E}$ & $\mathrm{Y}_{203}$ & $2 \mu \mathrm{m}$ to $>25 \mu \mathrm{m}$ & 91,5 & 1 & 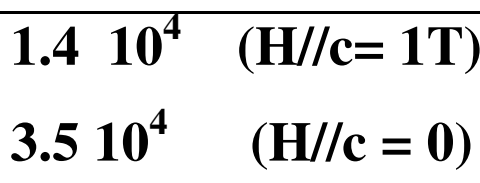 \\
\hline
\end{tabular}

Table 2

\begin{tabular}{|c|c|c|c|c|c|}
\hline YBCO & Type of material & Low angle GB & Large angle $\mathbf{G B}$ & Special $\sum \mathbf{G B}$ & $\mathbf{J}_{\mathbf{c}} \mathbf{A} / \mathrm{cm}^{2}$ \\
\hline
\end{tabular}




\begin{tabular}{|c|c|c|c|c|c|c|c|c|c|}
\hline system & & & \multicolumn{2}{|c|}{$<10^{\circ}$} & \multicolumn{2}{|c|}{$10^{\circ}<\theta<90^{\circ}$} & \multicolumn{2}{|c|}{$20^{\circ}<\theta<90^{\circ}$} & \\
\hline \multirow{4}{*}{$\begin{array}{l}\text { Solid-solid } \\
\text { diffusion } \\
\text { reaction }\end{array}$} & & & Clean & Degradated & Clean & Degradated & Clean & Degradated & \\
\hline & \multicolumn{2}{|c|}{$\begin{array}{c}\text { Bulk classic } \\
\text { Ceramic }\end{array}$} & Yes & No & Some & Some & Some & Some & $\mathrm{Jc}<10^{3}$ \\
\hline & \multicolumn{2}{|c|}{$\begin{array}{l}\text { Thin films grown on single } \\
\text { crystal substrate } \\
\mathrm{SrTiO}_{3}, \mathrm{MgO}\end{array}$} & Yes & No & Yes & No & Yes & No & $10^{6}<\mathrm{Jc}<10^{7}$ \\
\hline & \multicolumn{2}{|c|}{$\begin{array}{c}\text { GB thin film grown on } \\
\text { bicrystal } \\
\mathrm{SrTiO}_{3} \\
\end{array}$} & Yes & No & Yes & No & Yes & No & $\mathrm{Jc}=\mathrm{f}(\alpha)$ \\
\hline \multirow{5}{*}{$\begin{array}{l}\text { Solid-liquid } \\
\text { diffusion } \\
\text { reaction }\end{array}$} & \multicolumn{2}{|c|}{ Single crystal : Twins (110) } & ----- & ---- & ---- & ----- & Yes & ----- & $10^{5}<\mathrm{Jc}<10^{6}$ \\
\hline & \multicolumn{2}{|c|}{ Bulk bicrystals } & Yes & ----- & Yes & ----- & Yes & ----- & $\mathrm{J} c=\mathrm{f}(\alpha)$ \\
\hline & $\begin{array}{c}\text { Melt- } \\
\text { textured } \\
\text { Without } \\
\text { substrate }\end{array}$ & $\begin{array}{c}\text { under } \\
\text { magnetic field }\end{array}$ & Some & Some & Some & Some & ----- & ----- & $10^{4}<\mathrm{Jc}<10^{5}$ \\
\hline & \multirow{2}{*}{$\begin{array}{c}\text { Melt- } \\
\text { textured } \\
\text { with } \\
\text { substrate }\end{array}$} & $\begin{array}{c}\mathrm{Y}_{2} \mathrm{O}_{3} \text { ceramic } \\
\mathrm{Y}_{2} \mathrm{BaCuO}_{5} \\
\text { Ceramic } \\
(\mathbf{2 1 1}) \\
\end{array}$ & $\begin{array}{c}---- \\
\end{array}$ & $\begin{array}{c}---- \\
\end{array}$ & $\begin{array}{l}\text { Some } \\
\text { Some }\end{array}$ & $\begin{array}{l}\text { Some } \\
\text { Some }\end{array}$ & --- & $\begin{array}{ll}---- \\
\end{array}$ & $\begin{array}{l}10^{4}<\mathrm{Jc}<10^{5} \\
10^{4}<\mathrm{Jc}<10^{5}\end{array}$ \\
\hline & & $\begin{array}{l}\text { Single crystal } \\
\text { MgO }\end{array}$ & Some & Some & No & Yes & ----- & ----- & $\mathrm{Jc} \cong 0$ \\
\hline
\end{tabular}


Table 3 